\title{
Avaliação do Conhecimento Extraído de Problemas de Regressão*
}

\author{
Walter Aoiama Nagai
}

Orientadora: Profa. Dra. Solange Oliveira Rezende

Dissertação apresentada ao Instituto de Ciências Matemáticas e de Computação - ICMC-USP, como parte dos requisitos para obtenção do título de Mestre em Ciências - Área: Ciências de Computação e Matemática Computacional.

USP - São Carlos

Agosto de 2000

* Trabalho realizado com o apoio financeiro do CNPq 
Este documento foi preparado com o formatador de textos $\mathrm{FT}_{\mathrm{EX}}$. O sistema de citações de referências bibliográficas bibTEX utilizando o padrão apalike.

Todos os direitos reservados (CWalter Aoiama Nagai 


\section{Dedicatória}

Dedico aos meus pais, irmãos $e$ a minha "batian", pelo carinho e confiança que sempre me passaram. 


\section{Agradecimentos}

A minha orientadora e professora Solange Oliveira Rezende, pela amizade e orientação dedicados durante o mestrado.

Aos meus pais, irmãos e avó pela compreensão e apoio incondicional dedicados a miṃ.

A minha noiva Cláudia pelo Amor e Apoio, principalmente, nas horas difíceis.

Aos amigos do mestrado, Chandler, Cláudio, Daniela, Enzo, Huei, Igor, Janaina, José Geraldo (Zé), Maristela, Renato, Robson e Willian (Will).

Aos amigos que tenho desde a graduação, Amaury, André, Danielle, Danival, Gedson, Hana, Ivone, Marcelo Cintra, Rogério, Said e Vanessa.

À grande família "todos-solange@icmc.sc.usp.br", Alex, Antônio, Cristiane, Gerson, Jaqueline, Luis Carlos, Luiz, Marcos Paula, Marcos Geromini, Naiara, Roberta, Tatiana, Thereza, Valmir e Vitor.

Aos professores que sempre confiaram em mim e no meu potencial, Fábio H. V. Martinez, Marcelo F. Siqueira e Ronaldo A. Ferreira.

Ao CNPq pelo auxílio financeiro desde a minha iniciação científica. 


\section{Resumo}

Data Mining refere-se ao processo de análise de dados e à aplicaçāo de algoritmos que, mediante limitações de eficiência computacional aceitáveis, são capazes de produzir uma relação particular de padrões a partir de grandes massas de dados [Fayyad, 1996a].

A utilização desse processo em problemas do mundo real consiste na classificação dos dados, sejam eles categóricos ou contínuos. Problemas envolvendo dados categóricos são comumente denominados de problemas de classificação, enquanto que os dados contínuos são denominados $\mathrm{de}_{\ell}$ problemas de regressãg. Problemas do mundo real consistem geralmente de problemas de regressão. Dessa forma, cresce o interesse em utilizar o processo Data Mining para extrair padrões de problemas de regressão. Além da extração, esses padrões devem ser posteriormente analisados segundo algumas medidas de avaliação de conhecimento para determinar se o padrão é preciso, compreensível ou de interesse ao usuário.

Para explorar esse processo de avaliação do conhecimento em problemas de regressão, são realizados, neste trabalho, experimentos com conjuntos de diferentes domínios e características utilizando o ambiente $\mathcal{R R}_{\text {Evaluation }}$.

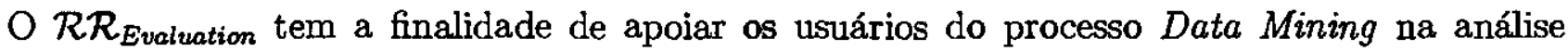

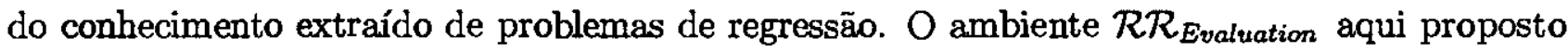
permite a utilização de diversas formas de avaliação da precisão utilizando as medidas MSE, MAD e NMSE. A compreensibilidade através da identificação do número de condições da regra e da função matemática envolvida, assim como algumas medidas de interessabilidade como GanhoMAD, LC e Q. 


\begin{abstract}
M refers to the process of data analysis and to the application of algorithms, within acceptable smputational efficiency limitations, which are able to find a particular pattern relationship om large quantities of data.
\end{abstract}

This process is used in real-world problems to classify data, whether it is categorical data or sontinuous data. Problems that involve categorical data are commonly called classification problems, while problems that involve continuous data are called regression problems. Realworld problems generally consist of regression problems. Because of this, there is an increasing interest in the use of DM to extract patterns from regression problems. Along with their extraction, these patterns should also be analyzed according to some knowledge evaluation measurements to determine if the pattern is precise, comprehensible or of interest to the user.

To explore this knowledge evaluation process in regression problems, experiments are executed on different domains with various characteristics using the $\mathcal{R} \mathcal{R}_{\text {Evaluation }}$ environment.

$\mathcal{R R}_{\text {Evaluation }}$ has as its main objective to support the users of the DM process in the analysis of the knowledge extracted from regression problems. The proposed environment makes it possible to use several forms of evaluating precision, using the MSE, MAD and NMSE measures. The comprehensibility can also be evaluated, by identifying the number of conditions in the rule and the mathematical function involved, as well as using some interestingness measures such as MADGain, LC and Q. 


\section{Sumário}

1 Introduçāo

2 Visāo Geral de Data Mining $\quad 4$

2.1 Considerações Iniciais . . . . . . . . . . . . . . . . . . . 4

2.2 Etapas do Processo de Data Mining . . . . . . . . . . . . 5

2.2 .1 Escolha da Atividade . . . . . . . . . . . . . . 6

2.2 .2 Escolha do Modelo/Algoritmo . . . . . . . . . . . . 7

2.2 .3 Preparação dos Dados . . . . . . . . . . . . . . 8

2.2 .4 Extração de Padrōes . . . . . . . . . . . . . . . . . 9

2.2 .5 Avaliação do Conhecimento Obtido . . . . . . . . . . . . . . . 10

2.3 Algumas Áreas de Apoio Relacionadas com Data Mining . . . . . . . . . . . . 11

2.4 Considerações Finais . . . . . . . . . . . . . . . . . . 13

3 Data Mining em Problemas de Regressão 14

3.1 Considerações Iniciais . . . . . . . . . . . . . . . . . . . . . 14

3.2 Problemas de Regressão . . . . . . . . . . . . . . . . 15

3.3 Aprendizado Baseado em Instâncias . . . . . . . . . . . . . . . . 15

3.3.1 Algoritmo do Vizinho mais Próximo . . . . . . . . . . . 16 
3.3.2 Locally Weighted Regression - LWR . . . . . . . . . . . . 17

3.4 Aprendizado Simbólico . . . . . . . . . . . . . 18

3.4 .1 Árvores de Regressão . . . . . . . . . . . . . . . . . . . . . . 19

3.4 .2 Regras de Regressão $\ldots \ldots \ldots \ldots \ldots$

3.5 Avaliação de Conhecimento em Problemas de Regressão . . . . . . . . . . . . 23

3.5 .1 Medidas de Precisão . . . . . . . . . . . . . . . . . 24

3.5.2 Medidas de Compreensibilidade $\ldots \ldots \ldots \ldots \ldots \ldots \ldots$

3.5.3 Medidas de Interessabilidade $\ldots \ldots \ldots \ldots \ldots \ldots$

3.6 Síntese das Medidas de Avaliação $\ldots \ldots \ldots \ldots$

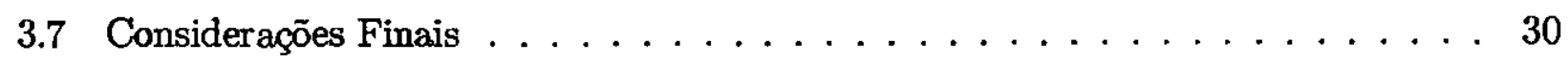

4 Ferramentas e Algoritmos Utilizados 31

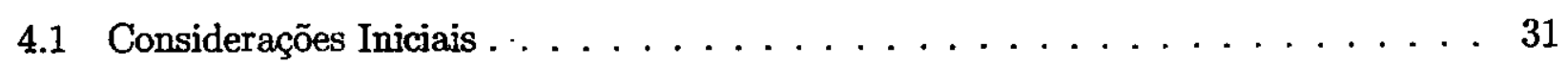

4.2 Algumas Ferramentas . . . . . . . . . . . . . 31

$4.2 .1 \operatorname{MineSet}^{T M} \ldots \ldots \ldots \ldots \ldots \ldots \ldots \ldots \ldots \ldots \ldots \ldots$

4.2.2 WEKA (Waikato Environment for Knowledge Analysis) . . . . . . 33

4.2.3 DMSK (Data-Miner Software Kit) $\quad \ldots \ldots \ldots \ldots \ldots$

4.3 Alguns Algoritmos usados para Problemas de Regressão . . . . . . . . . . . . 34

4.3 .1 Cubist $\ldots \ldots \ldots \ldots \ldots \ldots \ldots \ldots \ldots \ldots \ldots$

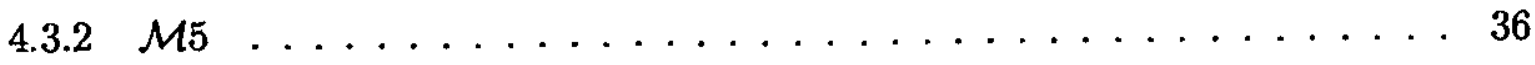

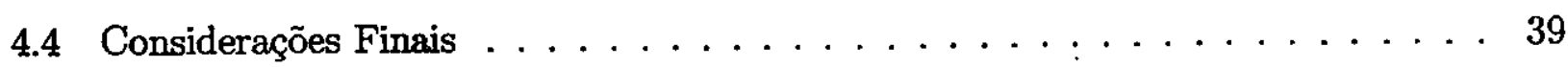

5 Ambiente de Avaliação de Regras de Regressão $\quad 40$

5.1 Consideraçōes Iniciais . . . . . . . . . . . . . 40 
5.2 Visão Geral do Ambiente $\mathcal{R}_{\mathcal{R}_{\text {Evaluation }}} \ldots \ldots \ldots$. . . . . . . . . . . 41

5.3 Descrição dos Dados de Entrada para o $\mathcal{R}_{\text {Evaluation }} \ldots \ldots$. . . . . . . . 42

5.3.1 Arquivo de Nomes ..................... . . 43

5.3 .2 Arquivo de Dados . . . . . . . . . . . . . . . . . . 44

5.3.3 Arquivo de $\operatorname{Regras} \ldots \ldots \ldots \ldots . \ldots . \ldots . \ldots 44$

5.4 Módulo de Conversão de Padrões para o formato da $\mathrm{B}_{R R} \ldots \ldots \ldots$

5.5 Módulo Rules . . . . . . . . . . . . . . . . . . . . 46

5.6 Módulo de Avaliação EvaluateRules . . . . . . . . . . . . . . . . . . . 48

5.6 .1 Guia Datasets . . . . . . . . . . . . . . . . 48

5.6 .2 Guia Graphics . . . . . . . . . . . . . . . . . . . . . . . 49

5.6 .3 Guia Statistical Analysis . . . . . . . . . . . . . . . 50

5.7 Considerações Finais $\ldots \ldots \ldots \ldots \ldots$

6 Experimentos Realizados $\quad 53$

6.1 Considerações Iniciais . . . . . . . . . . . . . . . . . . . 53

6.2 Dados Utilizados . . . . . . . . . . . . . . . . . . 53

6.2.1 Descrição Detalhada dos Conjuntos de Dados . . . . . . . . . . . . . . . 54

6.2.2 Síntese Geral das Características dos Conjuntos de Dados . . . . . . . . . 57

6.3 Descrição dos Experimentos Realizados . . . . . . . . . . . . . . . . . 58

6.3.1 Preparação dos Dados . . . . . . . . . . . . . . . . 59

6.3 .2 Extração de Padrões . . . . . . . . . . . . . . . . . . 60

6.4 Avaliação do Conhecimento Extraído . . . . . . . . . . . . . . 67

6.4 .1 Análise de Precisão . . . . . . . . . . . . . . . . . . . . . . 67

6.4 .2 Análise de Compreensibilidade . . . . . . . . . . . . 72 
6.4 .3 Análise de Interessabilidade $\ldots \ldots \ldots \ldots \ldots$

6.4 .4 Síntese das Medidas Avaliadas . . . . . . . . . . . . . . . 81

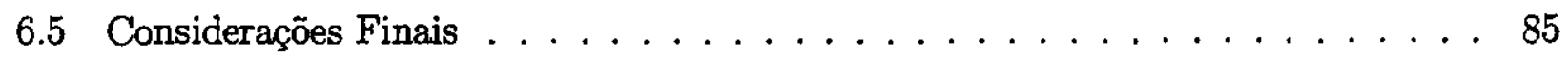

7 Conclusões e Trabalhos Futuros $\quad 87$ 


\section{Lista de Figuras}

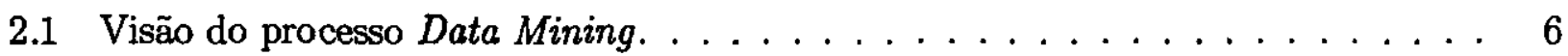

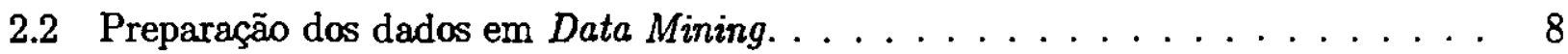

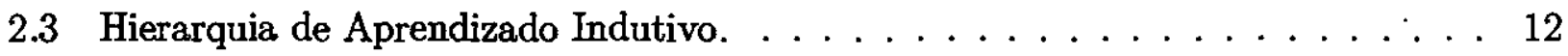

3.1 Exemplo de uma árvore de regressão. . . . . . . . . . . . . . . . . . . . 19

3.2 Regras geradas por um Sistema de Aprendizado. . . . . . . . . . . . 23

3.3 Exemplos cobertos pelas regras. . . . . . . . . . . . . . . . . . 24

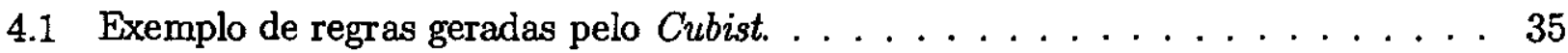

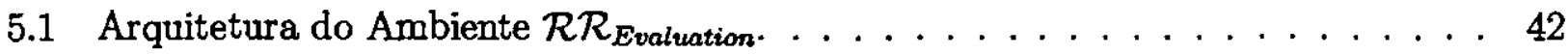

5.2 Exemplo do arquivo de nomes mpg names. . . . . . . . . . . . 43

5.3 Exemplo do arquivo de dados mpg.data. . . . . . . . . . . . . . . . 44

5.4 Exemplo do arquivo de regras de regressão mpg.rules. . . . . . . . . . . 44 45

5.5 Representação gráfica da árvore de regressão (a) e as regras geradas a partir da

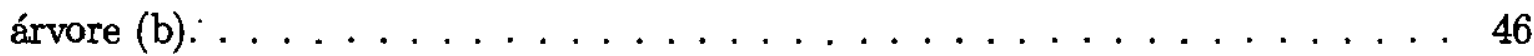

5.6 Regras de regressão traduzidas pelo $\mathcal{M}_{2}$ Rules. $\ldots \ldots \ldots \ldots$

5.7 Guia de observação dos dados no Módulo EvaluateRules do $\mathcal{R}_{\mathcal{R}_{\text {Evaluation }}}$. . . . 48

5.8 Guia de observação dos dados no Módulo EvaluateRules do $\mathcal{R}_{\mathcal{R}_{\text {Evaluation }}}$. . . . 49

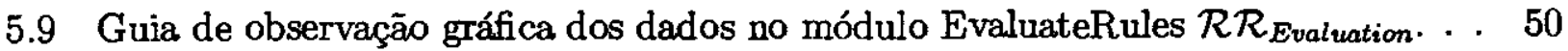


5.10 Gráfico plotado para a regra 6 selecionada. . . . . . . . . . . . . 50

5.11 Gráfico plotado em ordem crescente do valor real do atributo-meta para a regra 6 selecionada. . . . . . . . . . . . . . . . . . . 51

5.12 Guia das medidas de avaliação de conhecimento no módulo EvaluateRules do

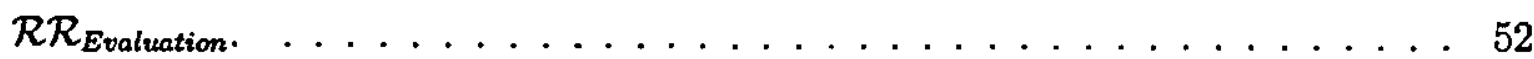

6.1 Dimensionalidade dos conjuntos de dados - Instâncias $\times$ Atributos. . . . . . 58

6.2 Diagrama de estados utilizado na realização dos experimentos. . . . . . . . . . 59

6.3 Regras obtidas pelo Cubist para o conjunto de dados CPU. . . . . . . . . . . 60

6.4 Regras obtidas pelo $\mathcal{M} 5_{2}$ Rules para o conjunto de dados CPU. . . . . . . . . 61 


\section{Lista de Tabelas}

2.1 Exemplo de um conjunto de dados no formato atributo-valor ou planilha. . . . . 8

3.1 Medidas de avaliação para problemas de regressão. . . . . . . . . . . . . . 29

5.1 Exemplo de um conjunto de dados de treinamento. . . . . . . . . . . . . 43

5.2 Gramática BNF que representa a sintaxe do arquivo de nomes. . . . . . . . . 4 43

5.3 Gramática BNF para a sintaxe do arquivo de nomes. . . . . . . . . . . . . 44

5.4 Gramática BNF usada para definir a sintaxe das regras da base $\mathrm{B}_{R R}$ do ambiente

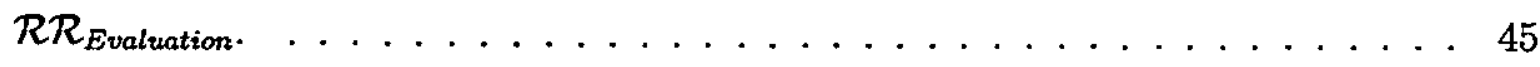

6.1 Descrição detalhada do conjunto de dados CPU. . . . . . . . . . . . . . . 55

6.2 Descrição detalhada do conjunto de dados Boston Housing. . . . . . . . . . . 55

6.3 Descrição detalhada do conjunto de dados MPG. . . . . . . . . . . . 56

6.4 Descrição detalhada do conjunto de dados Ozone. . . . . . . . . . . . . . 56

6.5 Descrição detalhada do conjunto de dados Permeability. . . . . . . . . . . . 57

6.6 Descrição detalhada do conjunto de dados PMGRN-455. . . . . . . . . . . 57

6.7 Síntese dos conjuntos de dados. . . . . . . . . . . . . . . . 57

6.8 Atributos e Preditores das regras obtidas pelo Cubist e pelo $\mathcal{M} 5$. . . . . . . 61

6.9 Condiçōes das regras obtidas pelo Cubist para o conjunto de dados Boston Housing. 62 
6.10 Funções matemáticas para regra obtida pelo Cubist no conjunto de dados Boston Housing. . . . . . . . . . . . . . . . . . . . . . . . 66 62

6.11 Condições das regras obtidas pelo $\mathcal{M} 5$ para o conjunto de dados Boston Housing. 62

6.12 Funções matemáticas para regra obtida pelo $\mathcal{M} 5$ no.conjunto de dados Boston Housing. . . . . . . . . . . . . . . . . . . . . . 63

6.13 Condições das regras obtidas utilizando os algoritmos Cubist e $\mathcal{M} 5$ no conjunto de dados MPG. . . . . . . . . . . . . . . . . . . . . . . 66 63

6.14 Equações matemáticas para cada regra obtida pelo algoritmo Cubist no conjunto de dados MPG. . . . . . . . . . . . . . . . . . . . . 63

6.15 Equações matemáticas para cada regra obtida pelo algoritmo $\mathcal{M} 5$ no conjunto de dados MPG. . . . . . . . . . . . . . . . . . . 63

6.16 Condições das regras obtidas utilizando os algoritmos Cubist e $\mathcal{M} 5$ para o conjunto de dados Ozone. . . . . . . . . . . . . . . . . . . . . . 64

6.17 Equações matemáticas das regras obtidas pelos algoritmos Cubist e $\mathcal{M} 5$ para o conjunto de dados Ozone. . . . . . . . . . . . . . . . . 64

6.18 Condições e equações das regras obtidas pelos algoritmos Cubist e $\mathcal{M} 5$ para o conjunto de dados Permeability - Cenário $1 \ldots \ldots \ldots$

6.19 Condições e equações das regras obtidas pelos algoritmos Cubist e $\mathcal{M} 5$ para o conjunto de dados Permeability - Cenário $2 \ldots \ldots \ldots \ldots 6$

6.20 Condições e equações das regras obtidas pelos algoritmos Cubist e $\mathcal{M} 5$ para o conjunto de dados Permeability - Cenário $3 \ldots \ldots 66$

6.21 Condições e equações das regras obtidas utilizando-se os algoritmos Cubist e $\mathcal{M} 5$ no conjunto de dados PMGRN-455-sexoM. . . . . . . . . . . . . 66

6.22 Condições e equaçōes das regras obtidas utilizando-se os algoritmos Cubist e $\mathcal{M} 5$ no conjunto de dados PMGRN-455-sexoF. . . . . . . . . . . 67

6.23 Condições e equações das regras obtidas utilizando-se os algoritmos Cubist e $\mathcal{M} 5$ no conjunto de dados PMGRN-455-total. . . . . . . . . . . . 67

6.24 Resumo dos resultados do Cubist. . . . . . . . . . . . . . . . . . 68 
6.25 Medidas de Precisão avaliadas no conjunto de dados CPU. . . . . . . . . . . 68

6.26 Medidas de Precisão avaliadas no conjunto de dados Boston Housing. . . . . . . 69

6.27 Medidas de Precisão avaliadas no conjunto de dados MPG. . . . . . . . . . . . 69

6.28 Medidas de Precisão avaliadas no conjunto de dados Ozone. . . . . . . . . 70

6.29 Medidas de Precisão avaliadas no conjunto de dados Permeability-cenario1. . . . 70

6.30 Medidas de Precisão avaliadas no conjunto de dados Permeability-cenario2. . . . 71

6.31 Medidas de Precisão avaliadas no conjunto de dados Permeability-cenario3. . . . 71

6.32 Medidas de Precisão avaliadas no conjunto de dados PMGRN455-sexoM. . . . . 71

6.33 Medidas de Precisão avaliadas no conjunto de dados PMGRN455-sexoF. . . . . 72

6.34 Medidas de Precisão avaliadas no conjunto de dados PMGRN455-total. . . . . . 72

6.35 Medidas de Compreensibilidade avaliadas no conjunto de dados CPU. . . . . . 73

6.36 Medidas de Compreensibilidade avaliadas no conjunto de dados Boston Housing. 73

6.37 Medidas de Compreensibilidade avaliadas no conjunto de dados MPG. . . . . . . 73

6.38 Medidas de Compreensibilidade avaliadas no conjunto de dados Ozone. . . . . . 74

6.39 Medidas de Compreensibilidade avaliadas no conjunto de dados PermeabilityCenario1. . . . . . . . . . . . . . . . . . . . 74

6.40 Medidas de Compreensibilidade avaliadas no conjunto de dados PermeabilityCenario2. . . . . . . . . . . . . . . . . . . . . 74

6.41 Medidas de Compreensibilidade avaliadas no conjunto de dados PermeabilityCenario3. . . . . . . . . . . . . . . . . . . 75

6.42 Medidas de Compreensibilidade avaliadas no conjunto de dados PMGRN455sexoM. . . . . . . . . . . . . . . . . . . . 75

6.43 Medidas de Compreensibilidade avaliadas no conjunto de dados PMGRN455-sexoF. 75

6.44 Medidas de Compreensibilidade avaliadas no conjunto de dados PMGRN455-total. 76 
6.45 Medidas de Interessabilidade avaliadas no conjunto de dados CPU. $\quad \ldots$. . . . 76

6.46 Medidas de Interessabilidade avaliadas no conjunto de dados Boston Housing. . 77

6.47 Medidas de Interessabilidade avaliadas no conjunto de dados MPG. . . . . . . . 78

6.48 Medidas de Interessabilidade avaliadas no conjunto de dados Ozone. . . . . . . . 78

6.49 Medidas de Interessabilidade avaliadas no conjunto de dados Permeability-cenario1. 79

6.50 Medidas de Interessabilidade avaliadas no conjunto de dados Permeability-cenario2. 79

6.51 Medidas de Interessabilidade avaliadas no conjunto de dados Permeability-cenario3. 79

6.52 Medidas de Interessabilidade avaliadas no conjunto de dados PMGRN455-sexoM. 80

6.53 Medidas de Interessabilidade avaliadas no conjunto de dados PMGRN455-sexoF. 80

6.54 Medidas de Interessabilidade avaliadas no conjunto de dados PMGRN455-total. 80

6.55 Síntese das medidas avaliadas para o conjunto de dados CPU. . . . . . . . . 81

6.56 Síntese das medidas avaliadas para o conjunto de dados Boston Housing. . . . . 82

6.57 Síntese das medidas avaliadas para o conjunto de dados MPG. . . . . . . . . . 82

6.58 Síntese das medidas avaliadas para o conjunto de dados Ozone. . . . . . . . 83

6.59 Síntese das medidas avaliadas para o conjunto de dados Permeability-Cenário1. . 83

6.60 Síntese das medidas avaliadas para o conjunto de dados Permeability-Cenário2. . 84

6.61 Síntese das medidas avaliadas para o conjunto de dados Permeability-Cenário3. . 84

6.62 Síntese das medidas avaliadas para o conjunto de dados PMGRN455-sexoM. . . 85

6.63 Síntese das medidas avaliadas para o conjunto de dados PMGRN455-sexoF. . . . 85

6.64 Síntese das medidas avaliadas para o conjunto de dados PMGRN455-total. . . . 85 


\section{Capítulo 1}

\section{Introdução}

Os avanços científicos e tecnológicos, juntamente com o aumento da capacidade de armazenamento a baixo custo e o desenvolvimento de novos conjuntos de métodos relacionados ao processamento de dados, têm colaborado para o crescimento da capacidade de gerar e coletar dados. A maioria dos dados disponíveis em empresas é de grande importância, pois deles podem ser induzidos conceitos ou características sobre uma determinada tecnologia ou produto, cuja análise possibilita, por exemplo, a escolha de melhores estratégias de negócios.

A preocupação cada vez maior das empresas públicas e privadas em adquirir novas tecnologias de processamento e armazenamento, além de considerar a informação e o conhecimento como seu maior patrimônio, tem direcionado várias pesquisas para o estudo do processo de transformação de dados armazenados em seus sistemas em conhecimento, o que pode proporcionar um auxílio efetivamente inteligente à tomada de decisão. A transformação de dados em conhecimento tem utilizado métodos eminentemente manuais para análise e interpretação de dados, o que torna $o$ processo de extração de conhecimento de bases de dados muitas vezes caro, lento e subjetivo, além de inviável em se tratando de grande volume de dados.

Recentemente, um processo tem tido considerável destaque na análise de dados oriundos de bases de dados com a finalidade de apoiar a tomada de decisão. Tal processo é conhecido como Data Mining, que é responsável por extrair padrões ou modelos implícitos embutidos em grandes conjuntos de dados [Fayyad, 1996].

Diante desse contexto, os pesquisadores da área de Inteligência Artificial (IA) estão cada vez mais investindo na investigação do processo de extração de conhecimento de dados com a finalidade de apresentar soluções úteis em diferentes domínios. Esses domínios podem ser divididos em dois grandes grupos, preditivos e descritivos, de acordo com o objetivo do processo. 
As atividades preditivas envolvem um processo indutivo capaz de generalizar casos com respostas conhecidas em uma linguagem que permite a predição de novos casos, já as descritivas são responsáveis pela identificação de comportamentos intrínsecos dos conjuntos de dados. A utilização de uma ou de outra atividade depende da existência ou não de um atributo-meta no conjunto de dados, isto é, caso o conjunto de dados não possua um atributo-meta é feita uma descrição desses dados, caso contrário, é feita uma atividade preditiva com o atributo-meta. Por sua vez a atividade preditiva envolve principalmente o tipo do atributo-meta (contínuo ou categórico); se o tipo do atributo-meta for categórico é feita uma classificaçāo, caso contrário é feita uma regressão [Weiss and Indurkhya, 1998].

Para ambas as atividades, um dos principais desafios de Data Mining consiste em analisar o conhecimento obtido levando em consideração, além da sua precisão, a avaliação da compreensibilidade e interessabilidade [Silberschatz and Tuzhilin, 1995]. Dentro desse contexto de avaliação de conhecimento obtido em Data Mining, muitas pesquisas foram realizadas com o intuito de apoiar os usuários na identificação de um conhecimento novo ou útil. Entretanto, muitas dessas pesquisas estão direcionadas a problemas que possuem atributos-meta categóricos, apesar de que muitos dos problemas do mundo real possuem atributos-meta contínuos [Weiss and Indurkhya, 1995].

Dessa forma, uma questão a ser ressaltada é que, embora existam várias técnicas e algoritmos disponíveis para analisar ou explorar um conjunto de dados, é necessário determinar o tipo de tarefa de aprendizado que o usuário deseja realizar, o tipo de dados disponíveis e, principalmente, o tipo de conhecimento que se deseja obter para então definir qual a metodologia, técnica e o algoritmo a serem utilizados. Este trabalho trata-se da extração de conhecimento de problemas de regressão enfatizando especialmente a avaliação dos resultados. Essa avaliação aborda algumas medidas, encontradas na literatura, bastante utilizadas para problemas de regressão e que foram propostas na tentativa de avaliar o conhecimento extraído desses problemas.

Neste trabalho é proposto um ambiente computacional denominado de $\mathcal{R}_{\mathcal{R}_{\text {Evaluation }} \text { tendo a }}$ finalidade de apoiar os usuários do processo Data Mining na análise do conhecimento extraído utilizando Data Mining preditivo em problemas de regressão. $O$ ambiente $\mathcal{R} \mathcal{R}_{\text {Evaluation }}$ aqui proposto permite a utilizaçāo de diversas maneiras de avaliar os resultados. Para tanto, são avaliados além do desempenho na regressão utilizando as medidas MSE, MAD e NMSE, a compreensiblidade através da identificação do número de condições da regra e da função matemática envolvida assim como algumas medidas de interessabilidade como GanhoMAD, LC e Q.

Para explorar esse processo de avaliação de resultados em problemas de regressão são realizados, 
neste trabalho, vários experimentos em diferentes domínios e com diferentes características

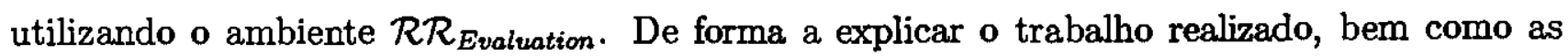
medidas de avaliação de conhecimento e o ambiente computacional $\mathcal{R}_{\mathcal{R}_{\text {Evaluation, }} \text {, a dissertação }}$ foi organizada da seguinte forma.

No Capítulo 2 é feita uma descrição geral do processo Data Mining, detalhando etapas do processo e algumas áreas do conhecimento envolvidas e utilizadas neste trabalho.

No Capítulo 3 é descrita a utilização de Data Mining em problemas de regressão. Săo descritas as abordagens de aprendizado mais utilizadas para esses problemas, bem como as representaçōes de conhecimento extraído mais utilizadas. Além disso, é feita uma descrição das medidas de avaliação de conhecimento extraído, focando-se principalmente em critérios de precisão, compreensibilidade e interessabilidade.

No Capítulo 4 são descritas as ferramentas e algoritmos utilizados nos experimentos realizados neste trabalho.

No Capítulo 5 é detalhado o ambiente computacional proposto para avaliação de regras de regressão denominado de $\mathcal{R} \mathcal{R}_{\text {Evaluation. }}$.

No Capítulo 6 são descritos os experimentos realizados, utilizando-se os algoritmos de apren-

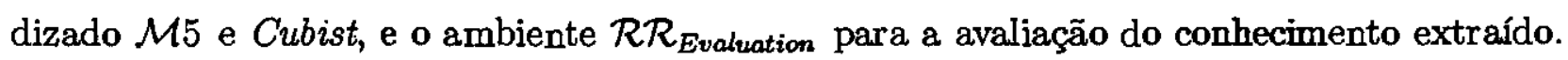

Por último, no Capítulo 7 são apresentadas as conclusões deste trabalho, bem como sugestões de trabalhos futuros. 


\section{Capítulo 2}

\section{Visão Geral de Data Mining}

\subsection{Considerações Iniciais}

Data Mining - DM refere-se à análise de dados e à aplicação de algoritmos que, mediante limitações de eficiência computacional aceitáveis, são capazes de produzir uma relação particular de padrões a partir de grandes massas de dados [Weiss and Indurkhya, 1998, Fayyad et al., 1996c]. DM pode ser considerado uma tecnologia que reúne técnicas vindas de algumas áreas do conhecimento, como Banco de Dados, Estatística e Inteligência Artificial, especialmente de Aprendizado de Máquina.

Algumas vezes, DM é confundido com ferramentas analíticas automáticas que auxiliam usuários nas análises e entendimento dos dados. Uma distinção significativa entre Data Mining e as ferramentas analíticas é que estas baseiam-se na abordagem de verificaçāo, na qual o usuário já possui algumas suposições ou hipóteses sobre um determinado relacionamento e então as utilizam para verificar ou refutar suas hipóteses. Por sua vez, Data Mining utiliza a abordagem baseada no descobrimento, na qual técnicas de reconhecimento de padrões, associação de atributos, agrupamento de valores, entre outras, são empregadas para determinar os relacionamentos implícitos existentes nos dados. Essas técnicas podem encontrar e analisar uma numerosa quantidade de relacionamentos e explicitar somente aqueles que são dominantes e excepcionais [Fayyad et al., 1996b].

Adicionalmente, Data Mining pode ser entendido como um processo constituído por etapas que auxiliam a utilização de técnicas de identificação de padrões nos dados, e por uma etapa posterior, na qual os padrões identificados são avaliados pelos usuários de Data Mining (especialista do domínio, analista e usuário final), a fim de verificar se representam um conhecimento 
já aprendido ou novo.

Para um entendimento desse processo, na Seção 2.2 são detalhadas brevemente as etapas que constituem o processo de extração de padrões. Na Seção 2.3 são detalhadas sucintamente algumas áreas do conhecimento que servem de base à aplicação de Data Mining. Por último, na Seção 2.4 são feitas as considerações finais deste capítulo.

\subsection{Etapas do Processo de Data Mining}

Data Mining pode ser considerado um processo direcionado a cumprir um determinado objetivo. A especificação desse objetivo está intrinsicamente relacionada aos dados a serem analisados e à forma como eles serão apresentados aos usuários. Alguns exemplos de objetivos consistem na classificação dos dados, no reconhecimento de relações entre atributos e na determinação de "grupos" de dados distintos.

A determinação de um ou mais objetivos para o processo de DM fica a cargo do analista e do especialista do domínio. Ressalta-se que Data Mining é um processo iterativo e interativo, isto é, a cada etapa do processo pode ser necessária uma interação entre os usuários de DM para a solução de problemas ou de dúvidas. Algumas vezes, o analista deve atuar, também, como mediador para os anseios e expectativas do especialista do domínio sobre o processo, para que os objetivos determinados sejam cumpridos e realizados a contento.

Na Figura 2.1 podem ser observadas detalhadamente as etapas de Data Mining, que são [Fayyad, 1996]:

1. Escolha da Atividade;

2. Escolha do Modelo/Algoritmo;

3. Preparação dos Dados;

4. Extração de Padrōes;

5. Avaliação do Conhecimento Obtido.

Essas etapas são explicadas sucintamente nas próximas subseções e observa-se que após um ciclo, pode-se voltar a qualquer etapa do processo Data Mining. 


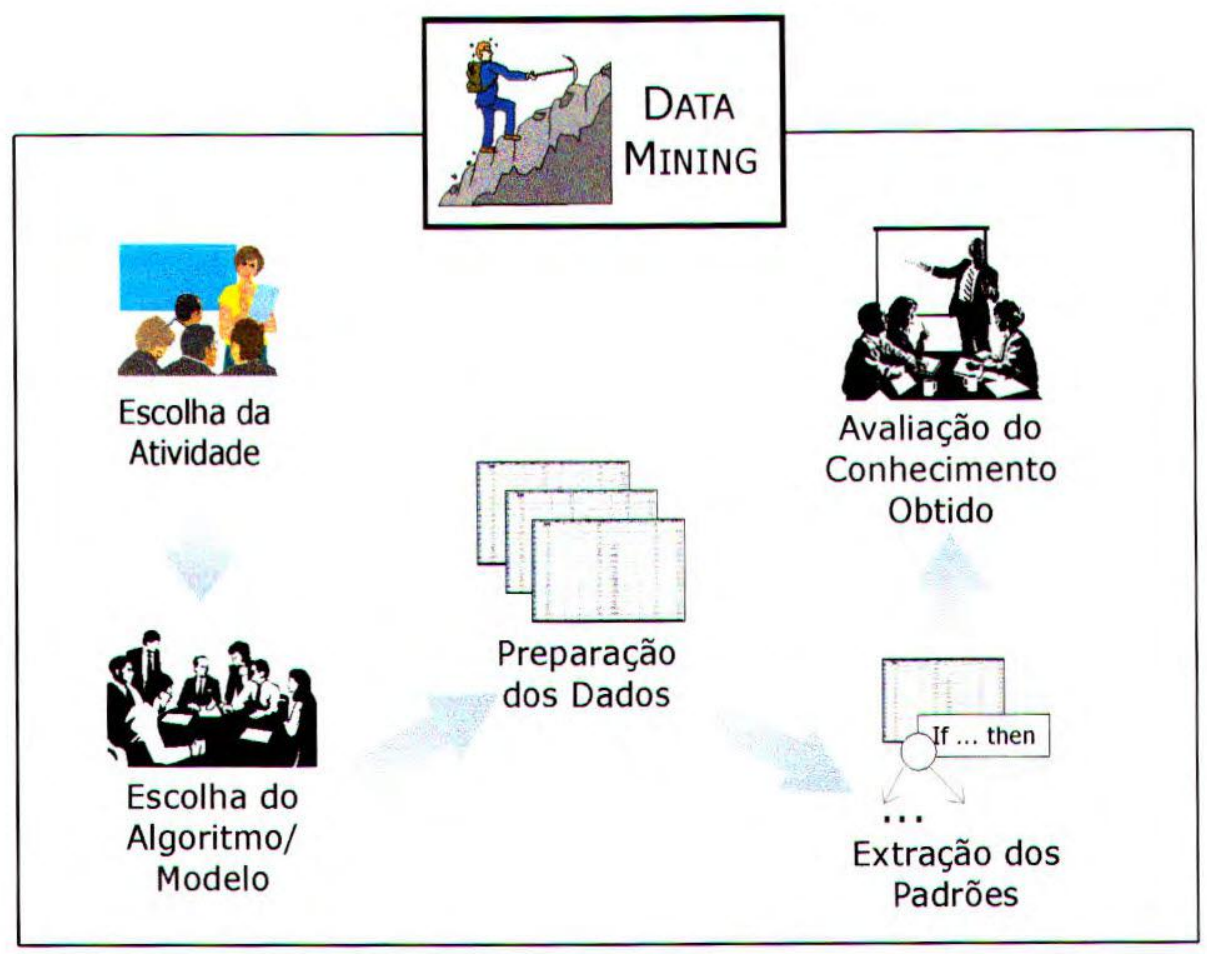

Figura 2.1: Visão do processo Data Mining.

\subsubsection{Escolha da Atividade}

A escolha de uma atividade de Data Mining é feita de acordo com algum objetivo especificado pelo analista, juntamente com o especialista. Sendo que essas tarefas estão organizadas em duas atividades principais: a predição e a descrição.

A atividade de predição, também conhecida como Data Mining Preditivo, consiste na predição do valor de um atributo $Y$ a partir de outros atributos $\left(X_{1}, X_{2}, \ldots, X_{n}\right)$, envolvendo um processo indutivo capaz de generalizar casos com respostas conhecidas em uma linguagem capaz de favorecer a predição de novos casos [Cheng, 1998]. Esta atividade pode ser auxiliada por ferramentas estatísticas e probabilísticas que suportam a formalização de problemas de predição [Elder and Pregibon, 1996].

Os principais algoritmos utilizados para predizer valores seguem geralmente padrões Estatísticos, de Redes Neurais ou de Aprendizado de Máquina Simbólico. A forma como o atributo-meta é representado determina o tipo de problema a ser tratado, isto é, se for um atributo-meta discreto é considerado um problema de classificação, caso contrário é considerado um problema de regressão. A solução de um problema de classificação refere-se à aplicação de um algoritmo 
capaz de mapear dados ou conjuntos de dados em categorias definidas, enquanto que em problemas de regressão refere-se à aplicação de um algoritmo capaz de mapear dados ou conjuntos de dados contínuos para um valor real, por meio de uma função matemática.

A atividade de descrição, ou Data Mining Descritivo, consiste na identificação de comportamentos intrínsecos dos conjuntos de dados. Sendo que esses dados não possuem um atributo-meta especificado como acontece na atividade de predição. Os comportamentos ou modelos descritos a partir dos dados são avaliados pelo especialista do domínio/analista, com a finalidade de identificar comportamentos excepcionais ou de senso comum. Alguns dos problemas de descrição mais encontrados são: Clustering e Sumarização.

Cada uma dessas atividades possuem técnicas, métodos ou algoritmos a serem utilizados para a resolução e avaliação do conhecimento extraído. No Capítulo 3 são detalhadas algumas das técnicas existentes para problemas de regressão que é o foco principal desse trabalho.

\subsubsection{Escolha do Modelo/Algoritmo}

Um modelo consiste na modelagem representativa dos padrões extraídos dos dados que será apresentado aos usuários do processo. Esse modelo deve ser capaz de apresentar algumas características desejáveis, tais como: a função e a sua representação. A função do modelo é determinada de acordo com o objetivo estabelecido de DM, por exemplo, se o objetivo for predizer um atributo-meta é escolhida a tarefa de classificação ou regressão.

A representação do modelo consiste na linguagem gerada por algum algoritmo para representar conceitos aprendidos a partir dos dados. Esta representação, geralmente, determina a flexibilidade do modelo na representação dos dados e a interpretabilidade do modelo em termos humanos. Uma representação muito utilizada por ser facilmente interpretável é a árvore de decisão, pois a sua estrutura (árvore binária) é facilmente entendida.

Outros tipos de representações de linguagem para modelos podem ser encontrados na literatura, entre os quais: árvores e regras de decisão [Quinlan, 1993a, Kerber, 1991, Clark and Niblett, 1989], modelos não-lineares (e.g. redes neurais) [Haykin, 1994], instance-based (e.g. métodos nearestneighbor [Ling and Wang, 1997, Kibler and Aha, 1988]), modelos de dependência probabilística ou inferência bayesina (e.g. redes Bayesianas) [Heckerman, 1997a, Heckerman, 1997b]. 


\subsubsection{Preparação dos Dados}

A etapa de preparação de dados é muito importante, pois atributos (features) ou instâncias não preparadas podem levar a resultados errôneos em Data Mining. Algumas transformações/reduções nos dados podem ser necessárias para que os mesmos possam estar adequados para serem utilizados pelos algoritmos. As tarefas de preparação de dados, mostrados na Figura 2.2, são executadas de acordo com a necessidade de preparação específica a cada algoritmo de extração de padrões. Por exemplo, tratamento de dados ausentes no conjunto de dados.

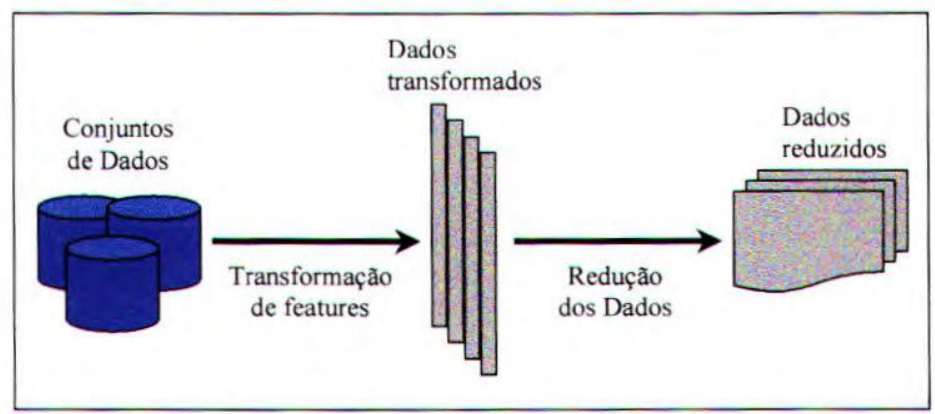

Figura 2.2: Preparação dos dados em Data Mining.

Segundo Weiss, é necessário que os dados transformados/reduzidos estejam organizados em um formato passível de ser processado pelos algoritmos [Weiss and Indurkhya, 1998]. Um exemplo de organização dos dados é mostrado na Tabela 2.1. Apesar desta organização limitar a visão real, é uma forma eficiente para que a maioria dos algoritmos possam processar os dados. Na Tabela 2.1 as colunas $f_{1}, \ldots, f_{k}$ são as features, $v_{1,1}, \ldots, v_{n, k}$ são os valores das features e $v_{1, k+1}, \ldots, v_{n, k+1}$ são os valores do atributo-meta. O número de instâncias, features e os valores das features constituem a dimensionalidade dos dados.

\begin{tabular}{|c|c|c|c|c|}
\hline \hline Casos & $f_{1}$ & $\ldots$ & $f_{k}$ & Atributo-meta \\
\hline \hline 1 & $v_{1,1}$ & $\ldots$ & $v_{1, k}$ & \\
\hline$\ldots$ & $\ldots$ & $\ldots$ & $\ldots$ & $\ldots$ \\
\hline $\mathrm{i}$ & $v_{i, 1}$ & $\ldots$ & $v_{i, k+1}$ & \\
\hline$\ldots$ & $\ldots$ & $\ldots$ & $\ldots$ & $\ldots$ \\
\hline $\mathrm{n}$ & $v_{n, 1}$ & $\ldots$ & $v_{n, k}$ & \multicolumn{2}{c|}{$v_{n, k+1}$} \\
\hline \hline
\end{tabular}

Tabela 2.1: Exemplo de um conjunto de dados no formato atributo-valor ou planilha.

A transformação de features é responsável pela alteração dos valores de features do conjunto de dados. Essas transformações podem ser simples, por exemplo, trocando-se sexo (M ou F) para 0 ou 1, ou complexas. Algumas das transformações mais complexas são: normalização e 
uniformizaçāo [Weiss and Indurkhya, 1998]. A primeira é utilizada para reduzir valores de um atributo a proporçōes menores, podendo ser manipulados por métodos como Nearest Neighbor. Enquanto a segunda reduz a quantidade de valores distintos de uma feature do conjunto de dados.

A redução dos dados é responsável por tratamentos nas dimensões do conjunto de dados (instâncias $\times$ features $\times$ valores). A execução dessa etapa influencia, principalmente, no desempenho dos algoritmos a serem utilizados na extração de padrões e pode atuar de duas formas [Weiss and Indurkhya, 1998]: (i) instâncias e (ii) features. A redução de instâncias atua na seleção ou diminuição do número de instâncias, motivado principalmente por alguma limitação computacional do algoritmo a ser utilizado. Por outro lado, a redução de features pode atuar na retirada de features menos relevantes; na composiçāo de novas features a partir da combinação de outras features (Composição de features - Aprendizado Construtivo [Lee, 2000]) e na discretização (determinação de intervalos de valores contínuos em uma feature).

Além desses tratamentos especiais para melhorar a precisão/desempenho dos algoritmos na etapa de Data Mining, alguns outros métodos podem ser necessários para o tratamento de dados incompletos, séries temporais e de textos para Text Mining.

\subsubsection{Extração de Padrões}

Um algoritmo para extração de padrões para Data Mining consiste na determinação de três componentes básicos [Fayyad et al., 1996a, Fayyad et al., 1996b]:

- Modelo do Algoritmo: refere-se à especificação da função do modelo ou tarefa de aprendizado e à representação do modelo (subseção 2.2.2);

- Critérios de Avaliação do Modelo: consiste na avaliação de parâmetros de configuração e de modelos extraídos pelos algoritmos. Esses critérios são específicos de cada um dos algoritmos de aprendizado, por exemplo, a existência de mecanismos para evitar overfitting.

- Algoritmo de Aprendizado: está relacionada à escolha de um algoritmo para extrair modelos com seus parâmetros particulares. Alguns dos algoritmos mais utilizados encontrados na literatura são: ID3, $\mathcal{C N} 2, \mathcal{C} 4.5, \mathcal{C} 4.5$-rules, See5.0, Cubist, k-Nearest Neighbor, AutoClass, $\mathcal{B K} \mathcal{D}$, Redes Perceptron e Lazy Decision Table. 
Com essa combinação de componentes é possível avaliar as soluções dadas por um ou mais algoritmos de extração de padrōes para um determinado problema de um domínio.

\subsubsection{Avaliação do Conhecimento Obtido}

Retirados os padrōes nos conjuntos de dados, uma avaliação deve ser feita a fim de analisar o conhecimento obtido por meio de critérios de:

- Precisão;

- Compreensibilidade;

- Surpresa/Interessabilidade.

Esses critérios auxiliam o especialista/analista na análise e complexidade da representação dos padrões encontrados, podendo ajudar também na filtragem do que foi aprendido, eliminando o conhecimento espúrio ou conhecimentos b́bvios guiados pelo senso comum, preocupando-se também em evitar o overfitting.

O critério de precisão está intimamente ligado com a magnitude e a forma de verificação de erros de um algoritmo. Existem muitos critérios de precisão para problemas de classificação e regressão, por exemplo, para problemas de classificação um critério é a análise da matriz de confusão (confusion matrix), enquanto que para problemas de regressão podem ser utilizadas as medidas MSE (Mean Squared Error) ou a MAD (Mean Absolute Difference) [Merz, 1998].

O critério de compreensibilidade está ligado a conceitos subjetivos relacionados principalmente à facilidade de compreensão da regra e à capacidade exploratória dos padrões retirados pelos usuários do processo.

Por fim, o critério de interessabilidade envolve alguns conceitos subjetivos e objetivos, por exemplo, conceitos sobre o domínio e os interesses dos usuários. Considere um modelo $\mathcal{H}$ de regras retiradas de um conjunto de dados, uma regra $R_{i}$ pode interessar mais a um usuário do que a regra $R_{k}$, que inclusive pode ser de interesse para outro usuário. Deste modo, dois aspectos podem ser considerados: o princípio de inesperabilidade descrito em [Silberschatz and Tuzhilin, 1996] e o princípio de acionabilidade descrito em [Piatetsky-Shapiro and Matheus, 1994]. A medida de inesperabilidade avalia se as regras são excepcionais (não observadas) frente ao conhecimento do especialista do domínio e a medida de acionabilidade avalia se o usuário pode utilizar as regras para obter vantagem na aplicação da regra. 
Dependendo do resultado da avaliação, pode-se retornar a algumas etapas do processo Data Mining para uma nova extração de padrões. Além disso, alguns algoritmos ou ferramentas para Data Mining oferecem recursos que apóiam as avaliações dos resultados, tais como: visualização multidimensional, análises estatísticas, entre outras.

\subsection{Algumas Áreas de Apoio Relacionadas com Data Mining}

Algumas áreas do conhecimento estão intimamente relacionadas com o processo Data Mining, dentre elas: Banco de Dados, Estatística e Aprendizado de Máquina.

A área de Banco de Dados tem contribuído com crescentes melhorias no armazenamento de diversas informações, bem como na estruturação de seus Sistemas Gerenciadores de Bancos de Dados (SGBD's) possibilitando que qualquer tipo e quantidade de informações sejam armazenadas.

$\mathrm{Na}$ área de Estatística, foram desenvolvidos métodos capazes de avaliar hipóteses e determinar diferenças probabilisticas em oportunidades aleatórias [Uysal and Güvenir, 1999]. A teoria estatística formal suporta modelos de dados e métodos para predição. Porém, modelos estatísticos clássicos, tipicamente modelos lineares, assumem um conjunto pequeno de dados e podem ser interrompidos quando o conjunto torna-se grande. Um dos métodos estatísticos mais utilizados para a extração de conhecimento é a Inferência Bayesiana. Alguns trabalhos envolvendo Autoclass e as Redes Bayesianas para aplicação em DM podem ser vistos em [Padilha and Rezende, 1999, Rocha, 1998].

Uma importante área relacionada com Data Mining é a de Aprendizado de Máquina que estuda e desenvolve métodos computacionais apropriados para a aquisição de novos conhecimentos, novas habilidades e novas formas de organização do conhecimento já existente. A procura por generalizações/conceitos é feita pela análise dos dados, utilizando-se para isso os sistemas de aprendizado. Um sistema de aprendizado é um programa de computador que toma decisões baseado em experiências acumuladas contidas em casos resolvidos com sucesso [Weiss and Kulikowski, 1991].

Um sistema de aprendizado pode ser classificado dentro de duas categorias [Kubat et al., 1998]:

- sistemas black-box, os quais desenvolvem suas próprias representações de conceitos, por- 
tanto sua representação interna não pode ser facilmente interpretada por humanos e assim, tais sistemas não suportam nenhuma explicação do processo de reconhecimento;

- sistemas orientados a conhecimento, os quais permitem as representações simbólicas a respeito dos conceitos que são facilmente compreensíveis.

Neste trabalho o foco é nos sistemas de aprendizado orientados a conhecimento, que trabalham sob algum paradigma de aprendizado, tentando aprender conceitos ${ }^{1}$ a partir de um conjunto de exemplos que estão descritos em uma linguagem de descriçāo de exemplos.

Os principais paradigmas de aprendizado para esses sistemas são: Estatístico, Instance-based, Genético, Redes Neurais e Simbólico; e as estratégias de aprendizado estão divididas em: hábito, instrução, dedução, analogia e indução. Essas estratégias, bem como os paradigmas de aprendizado estão detalhados em [Batista, 1997].

Um tipo de aprendizado muito utilizado é o aprendizado indutivo, que efetua uma busca dentro de um espaço de hipóteses por aquela que se aproxima da resposta correta, utilizando-se para isso, generalizações (formação de conceitos) feitas dentro do conjunto de exemplos. $O$ aprendizado indutivo possui uma categorização quanto ao tipo de aprendizado, como pode ser observado na Figura 2.3 [Baranauskas and Monard, 2000]..

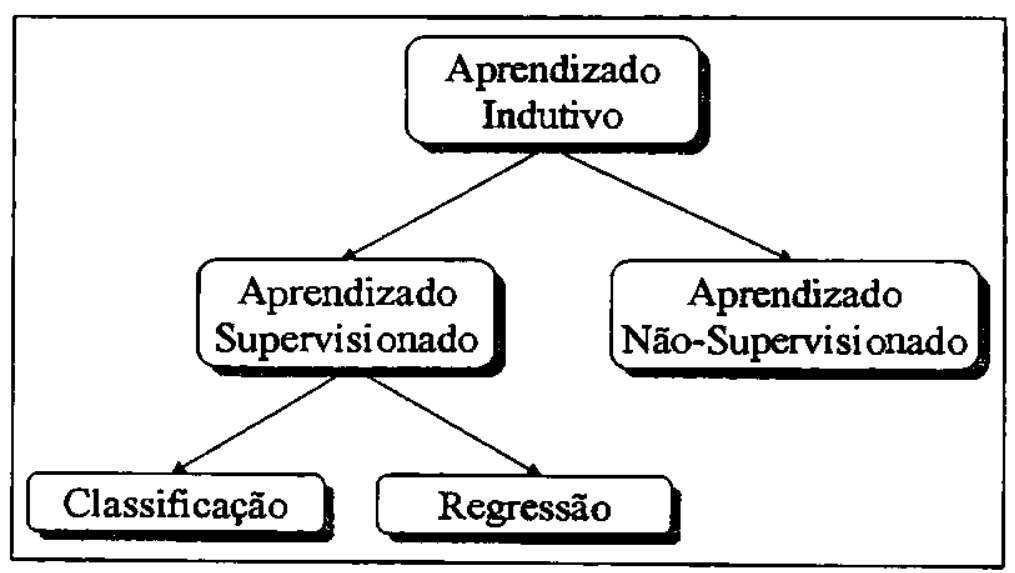

Figura 2.3: Hierarquia de Aprendizado Indutivo.

A diferença entre o aprendizado supervisionado e o não-supervisionado é a existência ou não de um atributo-meta a ser inferido, isto é, no aprendizado supervisionado o conjunto de instâncias consiste de valores de atributos e um atributo-meta com valor conhecido (classes ou valores

\footnotetext{
${ }^{1}$ Os conceitos aprendidos estão descritos em alguma linguagem de descrição de conceitos.
} 
reais), enquanto que no aprendizado não-supervisionado as classes são inferidas do próprio conjunto de instâncias. Deve-se salientar que o aprendizado não-supervisionado pode auxiliar o aprendizado supervisionado quanto à certificação ou identificação das classes de um conjunto de instâncias.

A própria natureza do aprendizado supervisionado é diretamente vinculada com a predição em Data Mining, pois a existência de um algoritmo de indução que consiga generalizar instâncias com respostas conhecidas possibilita a aplicação dos conceitos obtidos a novas instâncias ainda não-rotuladas. Deste modo, indutores de Aprendizado Supervisionado podem ser utilizados para a atividade de predição, como: indutores baseados em árvore [Quinlan, 1986, Breiman et al., 1984] e indutores baseados em regras [Clark and Niblett, 1989]. O primeiro induz descrições de conceitos na forma de uma árvore hierárquica que possui nós folhas com um valor predito. O segundo baseia-se em regras que obtém um conjunto de regras IF ... THEN, no qual as condições são testes lógicos dos atributos e a conclusão é um "rótulo" predito.

\subsection{Considerações Finais}

Neste capítulo foi realizada uma revisão do processo Data Mining e uma breve descrição de cada uma de suas etapas. Foram descritos também os problemas de predição, classificação e regressão, já que esses problemas englobam várias tarefas básicas de aprendizado com seus respectivos métodos que são oriundos de áreas como: Estatística e Aprendizado de Máquina. Cada uma dessas áreas contribuem no processo Data Mining, seja na preparação dos dados, na extração dos padrões ou na pré-avaliação do conhecimento obtido.

Como neste trabalho a atividade considerada é a de Data Mining Preditivo focando-se em problemas de regressão, no próximo capítulo é feita uma descrição detalhada desses problemas, detalhando as principais técnicas e as medidas de avaliação de conhecimento para esses problemas. 


\section{Capítulo 3}

\section{Data Mining em Problemas de Regressão}

\subsection{Considerações Iniciais}

Considerando-se o objetivo de predição nơ processo Data Mining, o próximo passo é a escolha do tipo do problema a ser investigado, seja classificação ou regressão. Neste trabalho o tipo do problema escolhido foi regressão. A regressão consiste na modelagem dos dados com a finalidade de predizer valores de um atributo-meta contínuo. Essa modelagem pode assumir diferentes formas de representação, com diferentes abordagens de aprendizado. Além disso, também é necessário utilizar medidas capazes de avaliar esses modelos com a finalidade de determinar o conhecimento útil ou de interesse.

Dentro desse contexto de avaliar problemas de regressão, o capítulo encontra-se organizado da seguinte forma: na Seção 3.2 é feita uma formalização dos problemas de regressão. Na Seção 3.3 são descritos os algoritmos com Aprendizado Baseado em Instâncias. Na Seção 3.4 são descritas as técnicas de Aprendizado Simbólico. Na Seção 3.5 são descritas as medidas de avaliação de conhecimento extraído referentes a problemas de regressão. Na Seção 3.6 é descrita uma síntese das medidas de avaliação; e por fim, na Seção 3.7 são feitas as considerações finais. 


\subsection{Problemas de Regressão}

O objetivo principal de Data Mining para problemas de regressão é encontrar um modelo $\hat{f}(X)$, baseado em amostras do conjunto de exemplos $X=\left\{x_{1}, x_{2}, \ldots, x_{n}\right\}$ [Torgo, 1999]. Esse modelo deve ser uma função que consiga mapear um vetor de entrada $X$ a um número real $\hat{Y}$ do atributo-meta $Y$. Deste modo, a técnica de regressão concentra-se em estimar ou predizer o valor do atributo-meta $Y$ baseado nos valores dos atributos $X^{1}$.

Em resumo, uma técnica de regressão deve ser capaz de relacionar os atributos $X$ com o atributo-meta $Y$ por meio de uma equação que pode ser descrita como:

$$
\hat{Y}=r\left(\beta_{i}, X_{i}\right)
$$

na qual, $r\left(\beta_{i}, X_{i}\right)$ é uma função que admite atributos $X_{i}=\left\{x_{1}, x_{2}, \ldots, x_{i}\right\}$ e pesos $\beta_{i}=$ $\left\{\beta_{1}, \beta_{2}, \ldots, \beta_{i}\right\}$ para cada atributo.

Diferentes técnicas podem ser utilizadas para representar $\hat{f}(X)$ e $\hat{Y}$. As próximas seçōes descrevem algumas abordagens que apóiam a resolução desse tipo de problema, dentre elas: o Aprendizado Baseado em Instâncias e Aprendizado Simbólico.

\subsection{Aprendizado Baseado em Instâncias}

O aprendizado baseado em instâncias (Instance-Based Learning - IBL) classifica um caso baseado em alguma similaridade com um outro caso cuja classe ou conceito é conhecido [Quinlan, 1993a, Weiss and Kulikowski, 1991]. De uma forma geral o aprendizado baseado em instâncias é composto de 3 componentes básicos: (i) um conjunto de exemplos ou instâncias de treinamento armazenadas em memória. Normalmente, este conjunto está no formato atributovalor; (ii) determinação de uma métrica para realizar comparações entre uma nova instância com instâncias mais "próximas" a ela. Essa métrica pode ser, por exemplo, a distância absoluta ou euclidiana; (iii) determinação de um número $k$ de instâncias próximas (vizinhas) ou similares que serão verificadas para a classificação de uma nova instância.

Normalmente, os algoritmos IBL possuem baixa capacidade exploratória, pois não são capazes de encontrar padrões que generalizam ou resumem critérios de decisão. Todas as explicações são baseadas em similaridades com casos armazenados em memória [Weiss and Indurkhya, 1998].

\footnotetext{
${ }^{1}$ Atributos que participam na predição de $\hat{Y}$ também são denominados de preditores [Uysal and Güvenir, 1999, Torgo, 1999].
} 
Um algoritmo que exemplifica o aprendizado baseado em instâncias é o Nearest Neighbor NN. Existe também uma outra técnica aqui apresentada, denominada de Locally Weighted Regression - LWR, que ao invés de classificar diretamente uma nova instância como o algoritmo NN, modela as instâncias vizinhas por meio de uma função matemática.

\subsubsection{Algoritmo do Vizinho mais Próximo}

Segundo Weiss, o algoritmo do Vizinho mais Próximo (Nearest Neighbor - NN) é um dos algoritmos de aprendizado mais simples, pois baseado em uma métrica de similaridade classifica um novo exemplo de acordo com um exemplo mais "próximo" ou similar [Weiss and Kulikowski, 1991].

Normalmente, um algoritmo NN utiliza como métrica de similaridade a distância euclidiana. Sendo que, essa métrica é limitada devido, principalmente, ao fato de classificar somente atributos contínuos. Na Equação 3.2 pode ser observada a métrica de distância euclidiana utilizada para comparar uma nova instância $x_{1}$ com uma instância $x_{2}$ armazenada.

$$
\Delta\left(x_{1}, x_{2}\right)=\sqrt{\left(x_{1,1}-x_{2,1}\right)^{2}+\left(x_{1,2}-x_{2,2}\right)^{2}+\ldots+\left(x_{1, d}-x_{2, d}\right)^{2}}
$$

em que $x_{i, j}$ é o valor do $i$-ésimo exemplo no $j$-ésimo atributo e $d$ é o número de atributos.

Para classificar um exemplo não visto $x_{t}$, a distância dele com todas as instâncias de treinamento $\Delta\left(x_{t}, x_{i}\right)$, deve ser calculada. O rótulo correspondente a $x_{t}$ é atribuído de acordo com a instância mais próxima.

Classificar um exemplo baseado somente em um caso similar pode acarretar em erros prematuros. Uma versão mais elaborada do algoritmo NN, denotada de $k$-Nearest Neighbor classifica um novo exemplo dentre as $k$ instâncias mais próximas fazendo-se a média entre elas.

Uma exigência dos algoritmos Vizinho mais Próximo é que as distâncias entre os atributos devem ser computáveis, ou seja, a métrica escolhida deve permitir manipulação de valores contínuos e categóricos. Atributos categóricos são considerados problemáticos porque nenhuma ordem é imposta aos seus valores. Por exemplo, considere um conjunto de dados em que um dos atributos a ser observado é a cor de uma peça automotiva.

Como considerar ou determinar que uma peça amarela é maior ou mais importante que uma peça marrom? 
No algoritmo PEBLS, por exemplo, existe uma métrica que calcula distâncias entre atributos categóricos, utilizando-se a Equação 3.3 [Uysal and Güvenir, 1999]:

$$
\Delta\left(x_{1}, x_{2}\right)=\sum_{i=1}^{d} \delta\left(x_{1, i}, x_{2, i}\right)^{2}
$$

na qual, $\delta\left(x_{1, i}, x_{2, i}\right)=\sum_{c=1}^{C}\left|P\left(c \mid x_{1, i}\right)-P\left(c \mid x_{2, i}\right)\right|^{k}, P\left(c \mid x_{1, i}\right)$ é a probabilidade de que o valor $v$ foi classificado na categoria $c$. Atributos contínuos são convertidos para atributos com valores categóricos, por meio de um processo de discretização. Porém, um dos problemas de discretizar é a possível "perda" da informação atribuída aos atributos contínuos.

Como esses algoritmos baseiam-se em casos previamente armazenados, a qualidade da predição pode ser comprometida por ruídos nos dados. A adequada seleção e preparação de features pode ser considerada uma ferramenta muito valiosa na obtenção de um melhor desempenho na capacidade preditiva dos algoritmos Vizinho mais Próximo. Para aplicações específicas, por exemplo, pode ser possível remover casos que não afetem o desempenho preditivo global.

Para conseguir obter uma boa capacidade preditiva, um processo de avaliação pode ser delineado da seguinte forma: o processo de avaliação inicia com o valor de $k$ igual a 1 até atingir o limite estipulado pelo usuário, erros de predição entre a nova instância e a "vizinha" são calculados para cada uma das instâncias. O número de vizinhos $k$ que obteve menor erro é o melhor valor para avaliar o conjunto de exemplos armazenados. Um comportamento peculiar deste algoritmo é o aumento do valor $k$ à medida que o número de exemplos cresce.

\subsubsection{Locally Weighted Regression - LWR}

LWR é similar à abordagem NN descrita anteriormente, principalmente em três características principais [Uysal and Güvenir, 1999]: (i) não existe uma fase de treinamento dos algoritmos para obtenção de regras, todo trabalho é feito durante a predição. Algoritmos NN e LWR são conhecidos como métodos de aprendizado lazy; (ii) as instâncias preditas são influenciadas por instâncias "perto" ou similares de instâncias já armazenadas e; (iii) as instâncias são representadas como pontos reais em um espaço p-dimensional.

Apesar dessas similaridades, NN prediz instâncias pela média das instâncias mais próximas, enquanto LWR constrói um modelo que compõem as instâncias mais próximas da nova instância a ser predita. Os modelos gerados são locais, ou seja, as instâncias mais próximas ou similares à nova instância possuem um peso maior enquanto que as menos similares ou mais distan- 
tes possuem um peso menor. Depois que um modelo é utilizado a uma nova instância ele é desconsiderado. Caso seja necessário predizer outra nova instância, um novo modelo deve ser construído [Uysal and Güvenir, 1999].

\subsection{Aprendizado Simbólico}

Um sistema de aprendizado simbólico utiliza-se de inferência indutiva para assim, aprender construindo representações simbólicas de um conceito através da análise de exemplos $\mathcal{L}$ e contraexemplos do conceito. No caso de problemas de regressão, pode-se utilizar a inferência indutiva para descrever essas representaçōes, função $\hat{f}(X)$, e utilizar uma função $\hat{Y}$ para determinar um valor contínuo.

As representações simbólicas estão tipicamente na forma de alguma expressão lógica, principalmente, árvores e regras de decisão [Félix, 1998]. Sendo que esses métodos de aprendizado simbólico podem ser tratados como:

- Proposicionais: esses métodos podem manipular enunciados declarativos, chamados proposições (ou sentenças) que aceitam somente respostas com valores verdadeiro e falso. É possível construir proposiçōes compostas através do uso de conectivos do tipo AND $(\wedge)$, OR $(\vee), \operatorname{NOT}(\neg)$, etc. Por exemplo, $($ cor $=$ vermelho $) \wedge($ forma $=$ circulo $)$ é uma disjunção de enunciados ou termos envolvendo valores dos atributos cor e forma. Essa disjunção de termos também é conhecida como FND (Forma Normal Disjuntiva). Existe uma outra notação proposicional denominada de FNC (Formal Normal Conjuntiva) que utiliza o operador OR (v).

- Relacionais: esses métodos estão representados pela Programaçāo Lógica Indutiva (PLI) orientada a construir programas lógicos - restritos às cláusulas de Horn. De maneira geral, PLI pode ser considerada como o uso de operadores lógicos para a representação explícita de problemas e de seu relacionamento com bases de conhecimento associadas, bem como a utilização de inferência lógica controlada para a solução efetiva desses problemas.

Nas próximas subseções são descritas algumas técnicas de representação de conceitos de Aprendizado Simbólico bastante utilizadas para tratar de problemas de regressão. 


\subsection{1 Árvores de Regressão}

As árvores de regressão sāo estruturas similares a árvores de decisão, distinguindo-se na forma como representam as classes nos nós-folhas [Quinlan, 1992]. Nas árvores de decisão, os nósfolhas possuem valores categóricos enquanto as árvores de regressāo, os nós-folhas possuem funções matemáticas que determinam valores reais como classe. Na Figura 3.1(a) pode ser vista uma árvore de regressão com atributos $x_{1}$ e $x_{2}$, e nós-folhas com valores 2, 8,5. Enquanto na Figura 3.1(b) pode ser observada a descrição textual de uma árvore de regressão clássica ${ }^{2}$, na qual $\mathrm{X}_{1}$ e $\mathrm{X}_{2}$ são os atributos e LM1 e LM2 são as constantes.

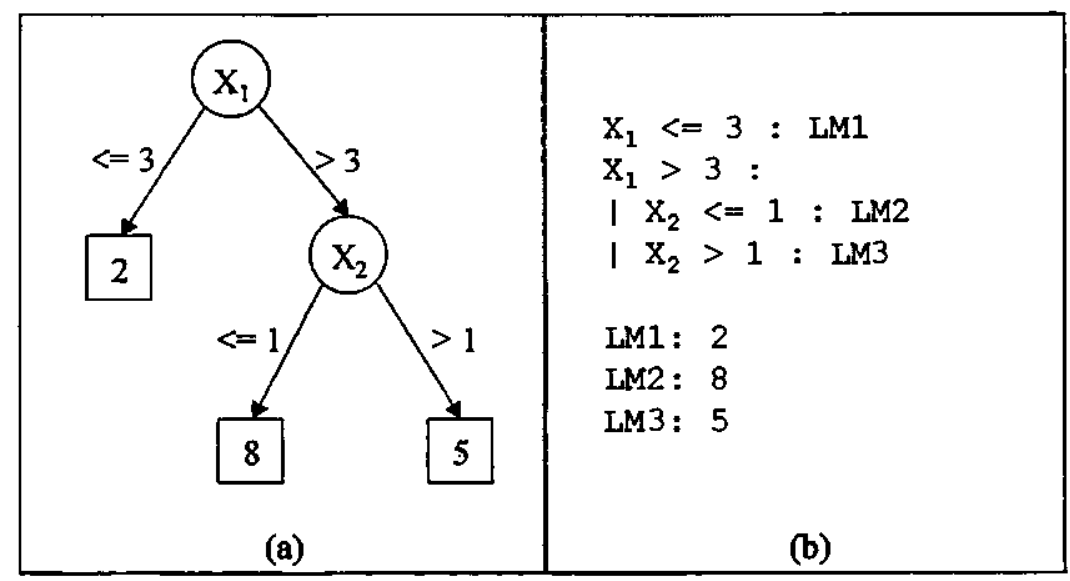

Figura 3.1: Exemplo de uma árvore de regressão.

Um primeiro fator a ser considerado na indução de árvores de regressão é o particionamento do conjunto de dados, por meio de um critério de seleção de atributos, dentro de regiōes disjuntas a cada nó da árvore. Algoritmos de indução de árvores de regressão, tais como: RETIS [Karalic, 1992], CART [Breiman et al., 1984], MARS [Friedman, 1990], $\mathcal{O C} 1$ [Murthy et al., 1994] e $\mathcal{M} 5$ [Frank et al., 1998, Wang and Witten, 1997, Quinlan, 1992], utilizam diferentes critérios de seleção para particionar os dados na tentativa de minimizar a variância do erro na predição do atributo-meta.

Um segundo fator é a construção de uma função matemática capaz de predizer, para cada subconjunto de dados, o atributo-meta. Os tipos de funções matemáticas mais comuns são: a função constante e a função linear. Normalmente, uma função constante no nó-folha é a média do atributo-meta de todas as instâncias percorridas do nó-raiz ao nó-folha. Enquanto uma função linear é uma equação formada por atributos $X_{i}$ (preditores) com pesos $\beta_{i}{ }^{3}$, que prediz

\footnotetext{
${ }^{2}$ Uma árvore de regressão clássica consiste dos nós-folhas rotulados com valores constantes.

${ }^{3}$ Alguns dos atributos que participam do conjunto de dados estāo presentes na função linear.
} 
o atributo-meta.

Uma árvore de regressão possui uma grande capacidade exploratória, podendo determinar atributos relevantes de um grande conjunto de dados por meio do critério de seleção escolhido, apesar que essa capacidade exploratória pode ser afetada pela complexidade da árvore [Wang and Witten, 1997].

A complexidade ou compreensibilidade de uma árvore pode ser expressa, por exemplo, pelo número de nós terminais (nós-folhas) da árvore. Um grande número de nós terminais pode influenciar no entendimento humano, sendo necessário assim, técnicas de retirada de nós ou poda para diminuir a complexidade. Existem várias formas de efetuar a poda de uma árvore, por exemplo, $\mathcal{M} 5$ utiliza testes de significância em cada nfvel da árvore a fim de identificar o nó-interior que pode ser removido. Os outros algoritmos de indução de árvore de regressão utilizam diferentes parâmetros de configuração on parâmetros de significância.

\subsubsection{Regras de Regressão}

Regras de regressão são semelhantes a regras de decisão [Witten and Frank, 1999], ou seja, manipulam condições na forma FND - <complex> e predizem uma classe ou atributo-meta. Sendo diferentes também das regras retiradas de uma árvore de regressão, pois as regras de regressão nāo são, obrigatoriamente, mutuamente exclusivas.

Alguns trabalhos encontrados na literatura, denotam que regras de regressão podem ser consideradas de duas formas quanto ao tratamento do atributo-meta:

1. Atributo-meta Discretizado: em [Weiss and Indurkhya, 1995] são denotadas regras de regressão que predizem um atributo-meta discretizado ${ }^{4}$. Dessa forma, algoritmos de classificação podem ser utilizados para determinar, por exemplo, árvores ou regras de decisão. Apesar de ser uma abordagem válida, ela modifica o problema de predizer o atributo-meta, ao determinar um intervalo em que pode estar o valor a ser predito [Torgo, 1997, Torgo, 1995].

2. Atributo-meta Contínuo: em vários trabalhos, o atributo-meta é predito por uma função matemática [Rulequest-Research, 2000, Torgo, 1999, Friedman, 1990].

Um exemplo do tratamento do atributo-meta (contínuo) discretizado pode ser encontrado em

\footnotetext{
${ }^{4} \mathrm{O}$ atributo-meta foi submetido a um processo de discretização (Torgo and ao Gama, 1997, Dougherty et al., 1995].
} 
[Weiss and Indurkhya, 1995], no qual um processo de discretização foi proposto, utilizando para isso, dois algoritmos: P-Class e o SWAP-1. O primeiro algoritmo (Algoritmo 3.1) é o responsável pela discretização dos valores contínuos do atributo-meta e o segundo algoritmo (Algoritmo 3.2) é responsável por melhorar a precisão de classificação, fazendo-se uma busca por melhores parâmetros ou condições das regras obtidas.

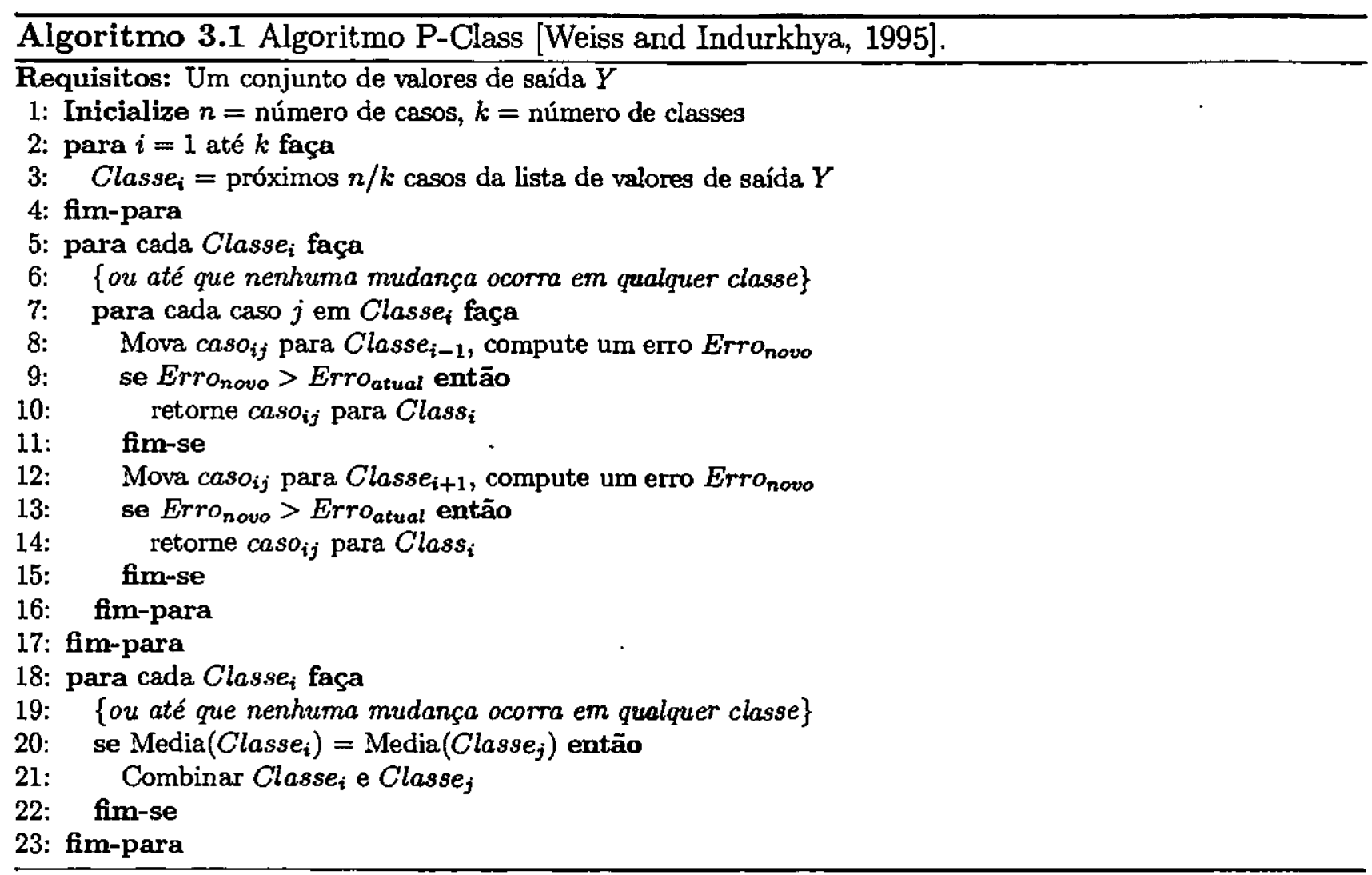

Para exemplificar o uso desses algoritmos, considere um conjunto de dados que tenha $Y$ como atributo-meta (considerando que os valores estão ordenados) e com os seguintes valores $Y=$ $\{1,2,4,4,6,6,8,8,9,10\}$. O algoritmo 3.1 faz uma divisão desse conjunto de dados em $k$ classes. Considerando que $k=3$, o conjunto de exemplos fica da seguinte forma:

$$
\underbrace{1,2,4,4}_{\text {classe1 }}, \underbrace{6,6}_{\text {dasse2 }}, \underbrace{8,8,9,10}_{\text {classe3 }}
$$

Realizada a discretização, um algoritmo de indução de regras de decisão, por exemplo $\mathcal{C N} 2$, pode ser utilizado para gerar as regras [Clark and Niblett, 1989]. Com as regras obtidas, o algoritmo SWAP-1 (Algoritmo 3.2) é utilizado para a otimização das mesmas, fazendo com que as regras sejam $100 \%$ preditivas. Apesar desse processo proposto por Weiss \& Indurkhya ser utilizado com o intuito de melhorar a capacidade preditiva de problemas de regressão, a 


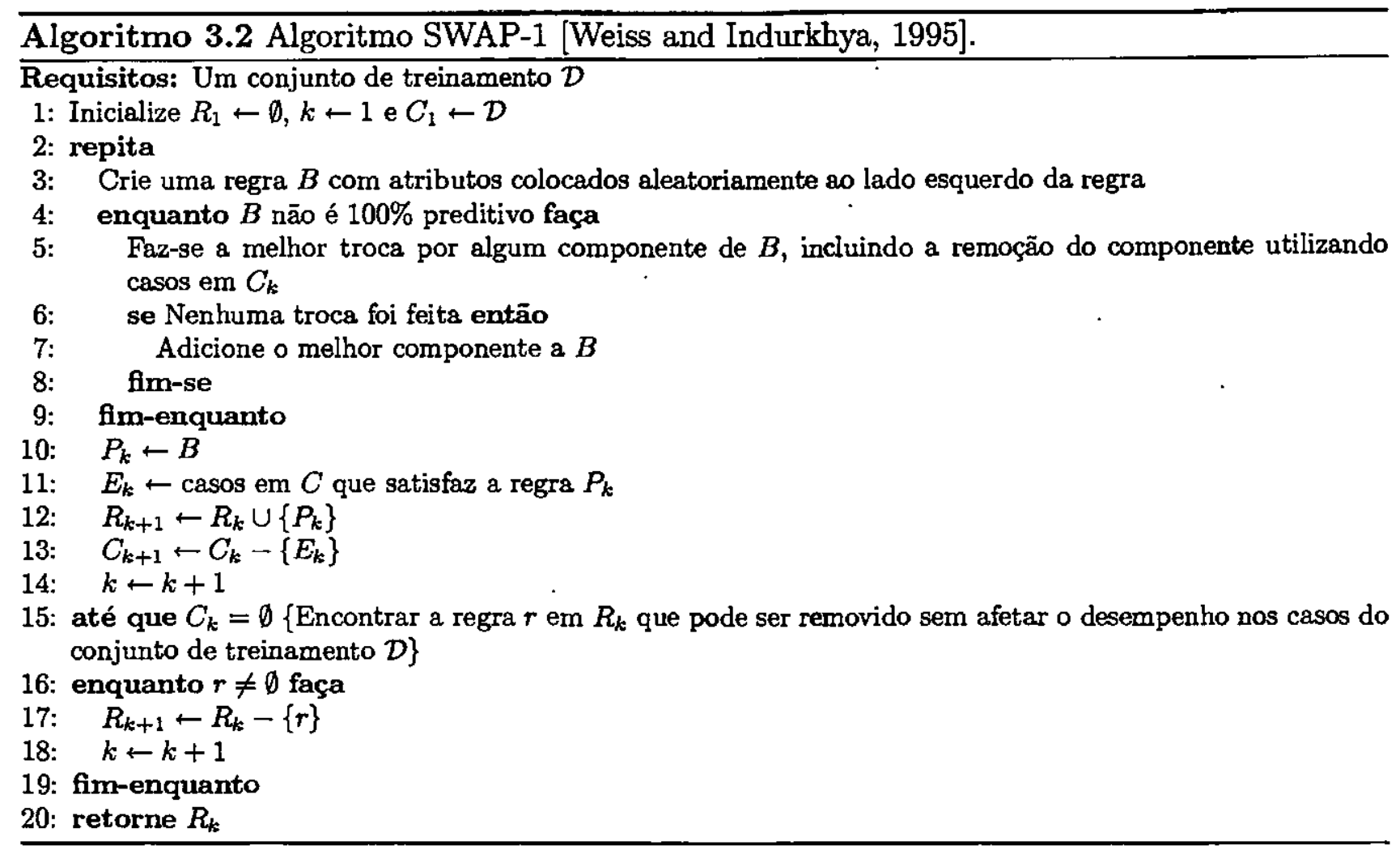

utilização do algoritmo P-Class e do SWAP-1 podem causar perdas de informação implícitas aos dados originais do problema [Torgo, 1997, Torgo, 1995].

Um exemplo do tratamento do atributo-meta contínuo, utilizado nesse trabalho, é a utilizada pelo Cubist [Rulequest-Research, 2000]. Uma regra de regressão possui condições na forma FND $-<$ condição $>$ e uma conclusão $<$ classe $=f\left(X_{i}\right)>$. Essas regras possuem uma forma geral igual à estrutura:

$$
\text { if }<\text { condição }>\text { then }<\text { classe }=f\left(X_{i}\right)>\text {, }
$$

na qual, $f\left(X_{i}\right)$ é uma função que possui como parâmetro uma lista de features $X_{i}=\left\{x_{1}, \ldots, x_{i}\right\}$ e <condição> são as condições da regra que assumem a forma $X_{i}$ op value, sendo que, $X_{i}$ é uma feature, op $\in\{=, \neq,<, \leq,>, \geq\}$ e value é um valor constante válido para a feature.

Pela estrutura das regras de regressão, pode-se observar que se um caso satisfaz todas as condições, o modelo linear $f\left(X_{i}\right)$ é apropriado para predizer o atributo-meta <classe>. Mas, se duas ou mais regras são escolhidas para um mesmo caso, normalmente, é feito uma média com os valores de resultados dos modelos lineares.

O Cubist permite a criação de modelos capazes de predizer o atributo-meta, por meio de um aprendizado LWR no conjunto de exemplos. Uma descrição mais detalhada do Cubist é feita no Capítulo 4. 


\subsection{Avaliação de Conhecimento em Problemas de Re- gressão}

Avaliar o conhecimento obtido pode envolver alguns problemas como:

- a dificuldade de fornecer conhecimento confiável sob todas as circunstâncias (por exemplo, quando há variações nos dados de origem do conhecimento adquirido, no tipo de representação desse conhecimento, entre outros);

- a falta de medidas de qualidade sempre confiáveis (por exemplo, que possam ser utilizadas em todos os domínios de aplicação) para servir de suporte aos usuários, tais como engenheiros de conhecimento ou engenheiros de processos.

Deste modo, pesquisadores têm buscado métodos de avaliação do conhecimento obtido a fim de procurar por conhecimento confiável e de interesse ao usuário. Essa avaliação está intimamente relacionada com a interpretação do conhecimento obtido.

Em alguns sistemas os padrões retirados estão contidos em uma "caixa-preta" (e.g. Redes Neurais ${ }^{5}$ ) ou representados explicitamente como um conjunto de regras $R_{1}, \ldots, R_{n}$ (e.g. Cubist) - Figura 3.2.

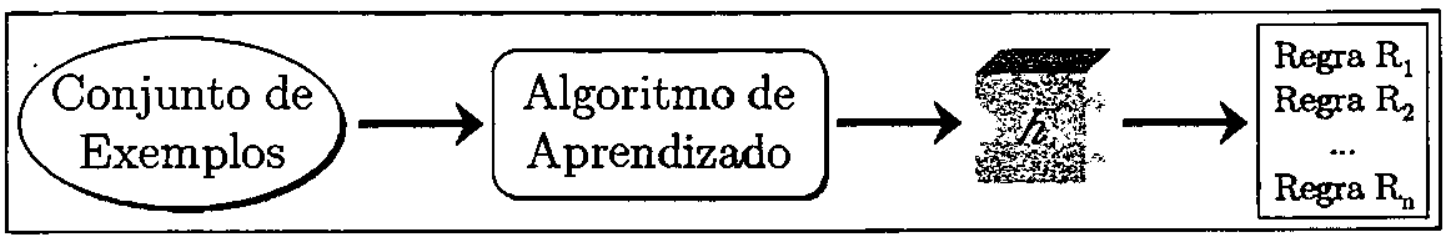

Figura 3.2: Regras geradas por um Sistema de Aprendizado.

Para efetuar uma avaliação dos padrões em Data Mining Preditivo, é necessária a retirada dos padrões que não possuem nenhuma validade ou utilização para um usuário. A razão disso é que, normalmente, um usuário possui algumas noções pré-concebidas do conhecimento do domínio. Conseqüentemente, quando um conjunto de regras é gerado a partir de algum conjunto de dados, o usuário desejaria saber [Liu and Hsu, 1996]: quais regras representam o meu conhecimento?, se não, qual parte do meu conhecimento está representado corretamente e qual não está? ou de que maneira as novas regras diferem do meu conhecimento anterior?

\footnotetext{
${ }^{5}$ Pesquisas recentes em Redes Neurais demonstram a extração de conhecimento a partir de uma rede treinada [Craven and Shavlik, 1999, Craven, 1996].
} 
Mas o que acontece quando um usuário não possui conhecimento do domínio necessário para identificar essas dúvidas? Por exemplo, o diretor de uma empresa deseja prever o total das vendas de seu produto no ano 2000 ou o lucro total gerado por seu produto. Para fazer essa predição são acumulados diversos dados com informaçộes de vendas do produto que serão utilizados para a geração de regras, conseguindo-se uma grande quantidade das mesmas. Mas, quais regras realmente interessam ao gerente? Determinar essas regras de interesse é também um problema para Data Mining [Liu and Hsu, 1996].

Nas próximas subseções são descritas medidas de interessabilidade para problemas de regressão, bem como medidas de precisão e compreensibilidade. Essas medidas são utilizadas nos experimentos realizados, descritos no Capítulo 6.

\subsubsection{Medidas de Precisão}

Regras de regressão, apesar de serem semelhantes às regras de classificação, não predizem um valor categórico definido e sim, um número real, envolvendo um grau de incerteza quanto à classe predita [Cheng, 1998]. Na Figura 3.3 podem ser observadas algumas regras de regressão que cobrem alguns exemplos do conjunto de treinamento (representados por $\bullet$ ), bem como as predições feitas (representadas por + ). Os pontos representados por $\bullet \mathrm{e}+$ representam os valores reais e preditos do atributo-meta de um problema de regressão.

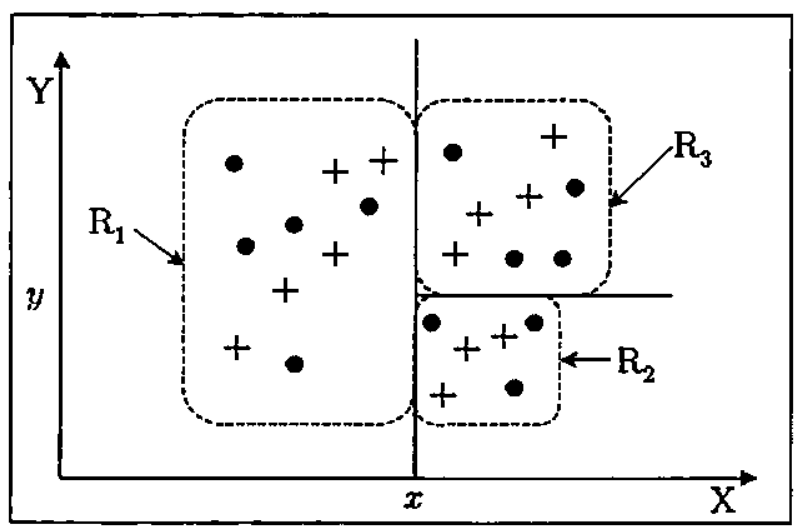

Figura 3.3: Exemplos cobertos pelas regras.

Normalmente, na avaliação de precisão do conhecimento obtido de problemas de regressão, utilizam-se três medidas para determinar a precisão dos modelos: MAD (Média da Diferença Absoluta), MSE (Média dos Erros ao Quadrado) e NMSE (MSE Normalizada) [Merz, 1998, Torgo, 1995]. 
A MAD (Equação 3.4) consiste na média da diferença (em módulo) entre os valores reais e preditos para um atributo-meta.

$$
\operatorname{MAD}(r)=\sum_{i=1}^{n} \frac{\left|y_{i}-r\left(\beta_{i}, x_{i}\right)\right|}{n}
$$

A MSE (Equação 3.5) consiste na média da diferença ao quadrado entre os valores reais e preditos para um atributo-meta. Essa medida, muitas vezes, é utilizada para minimizar o erro quanto à predição dos valores preditos ${ }^{6}$.

$$
\operatorname{MSE}(r)=\sum_{i=1}^{n} \frac{\left(y_{i}-r\left(\beta_{i}, x_{i}\right)\right)^{2}}{n}
$$

A NMSE (Equaçāo 3.6) consiste na normalizaçāo da MSE, pela diferença entre o valor real e o valor médio predito para o atributo-meta, ou seja, se o NMSE tende a 1, o modelo prediz mais o valor médio predito, caso contrário, ele prediz valores diferentes da média.

$$
\operatorname{NMSE}(r)=\sum_{i=1}^{n} \frac{\left(y_{i}-r\left(\beta_{i}, x_{i}\right)\right)^{2}}{\left(y_{i}-\bar{y}\right)^{2}}
$$

Nas Equaçōes 3.4, 3.5 e 3.6 o parâmetro $y_{i}$ é o valor do atributo-meta do exemplo de treinamento, $n$ é o número de exemplos de treinamento, $\bar{y}$ é o valor médio do atributo-meta e $r\left(\beta_{i}, x_{i}\right)$ é a função que calcula o valor predito para o exemplo de treinamento com os atributos $x_{i}$.

\subsubsection{Medidas de Compreensibilidade}

Medir o grau de compreensibilidade dos padrōes encontrados é uma tarefa dependente da capacidade de abstração do usuário. Dependendo do modelo obtido é necessário um critério diferenciado para avaliar a compreensibilidade.

Para árvores de regressão a compreensibilidade pode ser medida pelo número de nós da árvore. Árvores com muitos nós aumentam a complexidade de abstração da árvore [Ram, 1990]. Já para regras de regressão um fator de compreensibilidade é o número de condições da regra, visto que um grande número de condições pode dificultar o entendimento da regra.

\footnotetext{
${ }^{6} \mathrm{O}$ algoritmo $\mathcal{M} 5$, por exemplo, utiliza essa medida para minimizar o custo da escolhe do atributo para particionar o conjunto de dados.
} 
Um outro fator que influencia em ambas as técnicas é o número de termos existentes na função $f(X)$ (no caso das técnicas que predizem o atributo-meta utilizando-se uma função matemática), pois a existência de muitos termos pode dificultar a compreensão da função $f(X)$.

\subsubsection{Medidas de Interessabilidade}

A interessabilidade do conhecimento obtido é um dos tópicos em Data Mining que tem rec bido uma grande atenção por diversos pesquisadores. Piatetsky-Shapiro foi um dos primeir pesquisadores que discutiu a problemática da interessabilidade do conhecimento obtido e [Piatetsky-Shapiro, 1991]. Desde então, surgiram diversas propostas de medidas de interess bilidade, que incluem quatro abordagens:

1. Modelos de Regras: interessam somente as regras que "casam" com os modelos defini: dos com o especialista/usuário e o analista [Fu and Han, 1995, Klemettimen et al., 1994];

2. Cobertura de Regras Mínimas: algumas regras (um conjunto mínimo de regras que cobrem o maior número de exemplos do conjunto de dados) são apresentadas ao usuário [Toivonen et al., 1995];

3. Acionabilidade de Regras: as regras são consideradas interessantes, desde que o especialista/usuário possa obter vantagens ao utilizá-las [Silberschatz and Tuzhilin, 1995, Piatetsky-Shapiro and Matheus, 1994];

4. Inesperabilidade de Regras: as regras são consideradas interessantes se possuem um fator de inesperabilidade. A inesperabilidade pode ser imterpretada no sentido estatístico, como uma alta casualidade que aparece sob uma suposição ou hipótese independente ou sob algum bias que seja contrário às crenças do usuário [Liu and Hsu, 1996, Silberschatz and Tuzhilin, 1996].

Essas abordagens consideraram alguns fatores como [Liu and Hsu, 1996]: (i) a possível existência de um grande número de regras dificultando a análise por parte do usuário; (ii) a possível complexidade ao avaliar a regra, por exemplo, o número de condiçōes ou a estrutura de representação.

O primeiro fator deve-se ao tamanho do conjunto de dados analisado, pois dependendo das configurações dos algoritmos podem ser retiradas regras muito precisas que cobrem poucos 
exemplos, ou regras imprecisas que cobrem muitos exemplos. Enquanto o segundo fator devese a características da compreensão humana do usuário do processo.

Considerando as abordagens e os fatores de interesse, é comum encontrar na literatura diversas referências a medidas de interessabilidade para problemas de classificação, algumas delas em [Horst, 1999, Freitas, 1998]. Entretanto, medidas de interessabilidade para problemas de Regressão ainda estão em fase inicial de estudos, devido principalmente à incerteza de predizer o atributo-meta que se trata de um valor real. Desse modo, interessa ao usuário identificar os padrōes que tenham erros mínimos e que consigam generalizar o maior número de exemplos possível.

Para determinar a interessabilidade do conhecimento extraído em problemas de regressão, neste trabalho foram utilizadas algumas medidas de qualidade descritas em [Torgo, 1995]. Essas medidas de qualidade analisam o quão são precisas as regras, avaliando-se características de cobertura de exemplos e precisão na predição de um atributo-meta.

Desse modo, em [Torgo, 1995] são apresentadas medidas para avaliar a qualidade das regras especializadas. Uma regra especializada $R$ ' é construída variando-se operadores ou atributos de uma regra original $R$ com a intenção de se obter uma melhor precisão ${ }^{7}$. As medidas de avaliação de qualidade, GanhoMAD, LC e Q, quantificam algumas características de regras de regressão, tais como, precisão e representação da classe no formato de função matemática.

A medida GanhoMAD quantifica o ganho entre regras de regressão $r_{i}$ e $r_{k}$ diferenciadas pela MAD. A medida GanhoMAD é definida pela Equação 3.7:

$$
\text { GanhoMAD }(R)= \begin{cases}1-M_{A D} & , \text { se } R \text { for a regra original } \\ \frac{\mathrm{MAD}_{R}-\mathrm{MAD}_{R^{\prime}}}{\mathrm{MAD}_{R}} & , \text { se } \mathrm{R} \text { for uma regra especializada }\end{cases}
$$

na qual, $\mathrm{MAD}_{R}$ é o valor MAD da regra original R, enquanto que $\mathrm{MAD}_{R^{\prime}}$ é o valor da MAD da regra especializada $R$ '.

Para a utilização dessa medida é necessário que a MAD esteja normalizada, isto é, $\mathrm{MAD}_{n}=$ $\mathrm{MAD} / \mathrm{MAD}_{\text {maior }}$, na qual $\mathrm{MAD}_{\text {maior }}$ é a maior diferença entre um valor predito e real para a regra $r_{i}$. Essa normalização é nécessária para evitar a ocorrência de um ganho negativo.

Para exemplificar essa medida, considere $R_{1}$ e $R_{2}$ duas regras com $M_{A D} R_{1}=0,9$ e $M_{R_{2}}=$ $0,7\left(R_{2}\right.$ é uma regra especializada de $\left.R_{1}\right)$. Os valores de $\operatorname{GanhoMAD}\left(R_{1}\right)=1-0,9=0,1$ e GanhoMAD $\left(R_{2}\right)=(0,9-0,7) / 0,9=0,22$ representam os ganhos das regras quanto a MAD,

\footnotetext{
${ }^{7} \mathrm{Em}$ [Torgo, 1995] são feitos vários experimentos testando variaçōes entre operadores com os atributos da regra original para utilização no sistema $\mathcal{R}^{2}$.
} 
ou seja, a regra $R_{1}$ possui um "ganho" de precisão melhor que $R_{2}$ por ter um valor menor de GanhoMAD.

Uma outra medida de interessabilidade é a medida LC (Lost Coverage) quantifica a perda de cobertura dos exemplos entre duas regras R e R'. A LC definida pela Equação 3.8:

$$
\mathrm{LC}\left(\mathrm{R}^{\prime}\right)=\frac{\vec{C}_{R}-\bar{C}_{R^{\prime}}}{\bar{C}_{R}}
$$

na qual, $\bar{C}_{R}$ é o número de exemplos cobertos por uma regra $\mathrm{R}$, enquanto que $\bar{C}_{R^{\prime}}$ é o número de exemplos cobertos por uma regra especializada $R^{\prime}$. $O$ valor de LC para a regra original é o próprio número de exemplos.

Para exemplificar a medida LC, considere que $\bar{C}_{R}$ e $\bar{C}_{R^{\prime}}$ sejam o número de exemplos cobertos pela regra $R$ e $R^{\prime}$, com os valores 15 e 12 respectivamente. $O$ valor de $L C\left(R^{\prime}\right)=(15-12) / 15=$ 0,2 , ou seja, a perda de cobertura da regra especializada $R$ ' é de $20 \%$ em relação ao número de exemplos cobertos pela regra $R$.

Com o auxílio das medidas GanhoMAD e LC, uma medida de qualidade $Q$ da regra $\mathrm{R}$ pode ser calculada a partir de constantes (pesos) atribuídas a GanhoMAD e a LC, conforme a Equação 3.9:

$$
\mathrm{Q}(\mathrm{R})=\operatorname{GanhoMAD}(\mathrm{R}) * w_{\text {ganho }}+(1-\mathrm{LC}(\mathrm{R})) *\left(1-w_{\text {ganho }}\right)
$$

na qual, os pesos $w_{\text {ganho }} \mathrm{e}\left(1-w_{\text {ganho }}\right)$ representam um balanceamento entre a generalidade $\mathrm{e}$ a corretude da regra.

Se o valor de $w_{\text {ganho }}$ for alto, de acordo com a medida $Q$, as regras mais específicas (cobrem um pequeno número de exemplos) terão uma maior interessabilidade. Por outro lado, se o valor de $w_{\text {ganho }}$ for baixo, regras mais gerais que cobrem um número maior de exemplos serão escolhidas, diminuindo-se a precisão [Torgo, 1995]. A constante $w_{\text {ganho }}$ pode ser especificada pelo usuário determinando um intervalo $\left[w_{1} \ldots w_{2}\right]$ que consiste em um valor mínimo e máximo para minimizar ou maximizar o ganho, sendo que esses valores devem pertencer a um intervalo entre 0 e $1^{8}$. O cálculo de $w_{\text {ganho }}$ é calculado como mostra a Equação 3.10:

$$
w_{\text {ganho }}=w_{1}+\left(w_{2}-w_{1}\right) * \text { GanhoMAD }(\mathrm{R})
$$

\footnotetext{
${ }^{8} \mathrm{O}$ usuário também pode especificar um valor fixo $w_{\text {ganho }}$ ao invés de um intervalo.
} 
Uma medida complementar a $Q, L C$ e GanhoMAD para a verificação de interessabilidade de regras de regressão é a Completeza. A Completeza é uma medida que calcula a proporção de número de exemplos cobertos por uma regra $R$ no conjunto de $\mathrm{N}$ exemplos, como pode ser demonstrada na Equação 3.11:

$$
\text { Completeza }=\frac{\bar{C}_{R}}{N}
$$

Além de verificar precisão e interessabilidade das regras por meio das medidas de GanhoMAD, LC e Completeza, é importante, em se tratando de Data Mining verificar a compreensibilidade das regras. Neste trabalho, a compreensibilidade é tratada como a complexidade das regras, ou seja, número de atributos tanto na parte condicional da regra quanto na parte conclusiva.

\subsection{Síntese das Medidas de Avaliação}

As medidas utilizadas nos experimentos para avaliar os critérios de precisão, compreensibilidade e interessabilidade em problemas de regressão estão descritas na Tabela 3.1.

\begin{tabular}{c|c|c}
\hline \hline precisāo & compreensibilidade & interessabilidade \\
\hline MSE & Número de termos da condição da regra & LC \\
MAD & Número de termos da função $f(X)$ & $\mathbf{Q}$ \\
NMSE & & GanhoMAD \\
& & Completeza \\
\hline \hline
\end{tabular}

Tabela 3.1: Medidas de avaliação para problemas de regressão.

As medidas MSE, MAD e NMSE avaliam a precisão das regras de regressão quanto a predição do atributo-meta. As medidas de compreensibilidade avaliam a complexidade das condições e da conclusāo das regras e, por último, as medidas LC, Q, GanhoMAD e Completeza avaliam a interessabilidade em problemas de regressão.

Para exemplificar a utilização dessas medidas, considere duas regras $R_{1}$ e $R_{2}\left(R_{2}\right.$ é uma regra especializada de $R_{1}$ ) com as seguintes informações: $\bar{C}_{R_{1}}=18, \bar{C}_{R_{2}}=16, \mathrm{MAD}_{R_{1}}=0,35$, $\mathrm{MAD}_{R_{2}}=0,25, \mathrm{~N}=34$ (total de exemplos) e $w_{\text {ganho }}=0,65$ (65\% da precisão deve ser considerada). 


\begin{tabular}{|c|c|c|}
\hline $\mathrm{LC}\left(\mathrm{R}_{2}\right)=$ & $(18-16) / 18=$ & 0,11 \\
\hline GanhoMAD $\left(R_{1}\right)=$ & $1-0,35=$ & 0,65 \\
\hline GanhoMAD $\left(R_{2}\right)=$ & $(0,35-0,25) / 0,35=$ & 0,29 \\
\hline $\mathrm{Q}\left(\mathrm{R}_{1}\right)=$ & GanhoMAD $\left(\mathbf{R}_{1}\right)=$ & 0,65 \\
\hline $\mathrm{Q}\left(\mathrm{R}_{2}\right)=$ & $0,29 * 0,65+(1-0,11)^{*}(1-0,65)=$ &, 50 \\
\hline Completeza $\left(\mathrm{R}_{1}\right)=$ & $18 / 34=$ &, 53 \\
\hline Completeza $\left(\mathbf{R}_{2}\right)=$ & $16 / 34=$ & 0,47 \\
\hline
\end{tabular}

De acordo com as medidas utilizadas no exemplo, a regra $R_{2}$ possui uma qualidade $Q\left(R_{2}\right)=$ $0,50<Q\left(R_{1}\right)=0,65$. Dessa forma, sendo $Q\left(R_{1}\right)$ um valor de qualidade maior que $Q\left(R_{2}\right)$, a regra $R_{1}$ é considerada mais interessante que $R_{2}$. $R_{2}$ possui uma precisão menor e $R_{1}$ possui uma maior generalidade devido a Completeza $\left(R_{1}\right)=0,53>\operatorname{Completeza}\left(R_{2}\right)=0,47$.

\subsection{Considerações Finais}

Neste capítulo foram descritas algumas das técnicas mais utilizadas ao se trabalhar com problemas de regressão. Algumas delas possuem semelhanças com técnicas utilizadas para problemas de classificação.

Também foram descritas algumas medidas de avaliagaão de conhecimento obtido, como: precisão, compreensibilidade e interessabilidade, que determinam as regras de maior interesse ao especialișta/usuário do domínio, analisando-se características de regras de regressão.

Além de descrever essas medidas, é importante utilizá-las para obter resultados a fim de repassar ao usuário as regras mais interessantes. Dentro desse contexto, o ambiente de avaliação $\mathcal{R} \mathcal{R}_{\text {Evaluation }}$ (descrito no Capítulo 5) foi desenvolvido com o objetivo de avaliar o conhecimento obtido (em forma de regras de regressão) pelos algoritmos $\mathcal{M} 5$ e Cubist (descritos no próximo capítulo) nos experimentos realizados (Capítulo 6). 


\section{Capítulo 4}

\section{Ferramentas e Algoritmos Utilizados}

\subsection{Considerações Iniciais}

Nesse capítulo são descritas as ferramentas e os algoritmos utilizados para a realização dos experimentos descritos no Capítulo 6. Os algoritmos utilizados foram o $\mathcal{M} 5$ e $C u b i s t$, que extraem padrōes na forma de árvores e regras de regressão, respectivamente. As ferramentas utilizadas foram o MineSet ${ }^{T M}$, WEKA e DMSK que possuem recursos de visualização, manipulação e tratamentos dos conjuntos dos dados.

Este capítulo encontra-se organizado da seguinte forma: na Seção 4.2 estão descritas ferramentas de auxílio para DM Preditivo. Na Seçāo 4.3 são descritos os algoritmos utilizados; e por último, na Seção 4.4 são feitas as considerações finais do capítulo.

\subsection{Algumas Ferramentas}

No trabalho foram utilizadas algumas ferramentas disponíveis no Laboratório de Inteligência Computacional - LABIC/ICMC, que sāo o MineSet ${ }^{T M}$, WEKA (Waikato Environment for Knowledge Analysis) e o DMSK (Data-Miner Software Kit). Cada uma dessas ferramentas possui bibliotecas/funções que auxiliam o usuário em etapas do processo DM. Algumas das características principais dessas ferramentas são descritas nas próximas subseções. 


\subsubsection{MineSet $^{T M}$}

O MineSet ${ }^{T M}$ [Vanderberg, 2000] consiste de um software produzido pela Silicon Graphics que possui um conjunto de ferramentas que auxiliam o analista/especialista na exploração dos dados. Para esta funcionalidade, o MineSet ${ }^{T M}$ possui ferramentas de visualização e uma biblioteca, denominada $\mathcal{M L C}^{++}$, contendo alguns dos principais algoritmos de Aprendizado de Máquina.

A biblioteca $\mathcal{M L C}^{++}$foi projetada para auxiliar o usuário na seleção de algoritmos que executam tarefas específicas e/ou no desenvolvimento de novos algoritmos mais convenientes para outras tarefas. Além de ser uma biblioteca de classes em $\mathcal{C}^{++}$, provê utilitários e ferramentas que podem ser utilizadas isoladamente. Uma descrição mais completa dessa biblioteca pode ser encontrada em [Félix et al., 1998a, Kohavi et al., 1997, Kohavi et al., 1994].

No entanto, as ferramentas de visualização são as mais utilizadas por especialistas do domínio. Isso se deve a enorme capacidade exploratória que essas ferramentas oferecem, fornecendo visões dos dados e gráficos das estatísticas e dos resultados obtidos a partir da $\mathcal{M L C} C^{++}$.

De forma a diferenciar as ferramentas de visualização da biblioteca $\mathcal{M L C}^{++}$, a ferramenta MineSet $^{T M}$ está dividido em três módulos principais [Vanderberg, 2000]:

- Módulo de Controle Centralizado: consiste de uma interface gráfica chamada Tool Manager e um processo denominado Data Mover que é executado no servidor todas as vezes que o MineSet ${ }^{T M}$ é iniciado;

- Módulo de Ferramentas para Data Mining: consiste de 4 utilitários: (i) gerador de regras de associação; (ii) classificador e indutor de árvore de decisão; (iii) importância das colunas (atributos) e; (iv) classificador e indutor de evidência. Todas essas ferramentas estão na biblioteca $\mathcal{M L C}^{++}$;

- Módulo de Ferramentas de Visualização: consiste de 8 módulos de visualização: (i) visualizador de Árvore de Decisão; (ii) visualizador bidimensional e tridimensional; (iii) visualizador de mapas; (iv) visualizador de regras; ( $v$ visualizador de evidências da classe dentro de cada atributo; (vi) visualizador da base ou conjunto de dados; (vii) visualizador de estatísticas geradas pelo MineSet ${ }^{T M} \mathrm{e}$; (viii) visualizador difuso bidimensional e tridimensional.

O MineSet ${ }^{T M}$ tem como objetivo principal, a visualização multidimensional dos dados, auxiliando o usuário de Data Mining Preditivo a encontrar relaçōes ou padrões não observados. 
Em [Rezende et al., 1998] é descrito um trabalho focando-se na importância da Visualização no Processo de Extração de Conhecimento $-\mathcal{K D D}$, utilizando-se para isso, o software MineSet ${ }^{T M}$.

\subsubsection{WEKA (Waikato Environment for Knowledge Analysis)}

A WEKA [Witten and Frank, 1999] consiste de uma biblioteca contendo implementaçōes de alguns algoritmos de Aprendizado de Máquina para a utilização em problemas de Data Mining. Os algoritmos foram implementados na linguagem Java, podendo ser executados na maioria das plataformas existentes. Esses algoritmos podem ser utilizados diretamente nos conjuntos de dados ou a partir de uma aplicação Java escrita pelo usuário. Por ser uma biblioteca de algoritmos semelhante a $\mathcal{M L C}^{++}$, WEKA também pode ser utilizada para desenvolver novos algoritmos ou variantes dos algoritmos de Aprendizado de Máquina já implementados. Alguns dos algoritmos implementados na biblioteca WEKA são: J48 (versão Java do algoritmo C4.5), ID3, Naive Bayes, Regressão Linear e $\mathcal{M} 5$.

A WEKA possui implementações de algoritmos para diferentes paradigmas, entre eles, estatístico, instance-Based e proposicional. Ela também possui ferramentas que executam tarefas de preparação de dados, tais como: normalização de valores, discretização de valores contínuos e redução de valores e instâncias. Informações adicionais sobre a biblioteca podem ser encontradas em http://www.cs.waikato.ac.nz/"ml/weka/index.html.

\subsubsection{DMSK (Data-Miner Software Kit)}

O DMSK consiste de uma coleção de programas que implementam técnicas de Data Mining apresentadas em [Weiss and Indurkhya, 1998] e foi criado especialmente para atender um público diferenciado, entre eles, executivos, gerentes de negócios e desenvolvedores.

O DMSK é multiplataforma, pois existem versões para Windows, Unix ou Linux e Java. Todos os programas implementados consistem de ferramentas para preparação de dados, Text Mining e Predição. O DMSK possui programas de preparação de dados que permitem a redução, união, normalização e clusterização das instâncias e atributos, bem como a utilização de métodos de predição de classificação (Decision Trees, Decision Rules e Association Rules) e regressāo (Regressão Linear, Redes Neurais e Nearest Neighbor). Informações adicionais sobre DMSK podem ser encontradas em http://www.data-miner .com/dmsk.html. 


\subsection{Alguns Algoritmos usados para Problemas de Re- gressão}

Com base nas especialidades de cada técnica e o foco do trabalho centrado na extração de conhecimento, foram utilizados algoritmos para geração de regras e árvores de regressão. Dentre os algoritmos que possibilitam tais representações de conhecimento, foram utilizados o Cubist que gera regras de regressão e o $\mathcal{M} 5$ que gera árvores de regressão.

\subsubsection{Cubist}

O Cubist consiste de uma ferramenta para geração de regras não ordenadas para conjuntos de dados que têm como atributo-meta (ou classe) um valor contínuo [Rulequest-Research, 2000]. No Algoritmo 4.1 pode ser observada a indução de regras utilizada pelo Cubist.

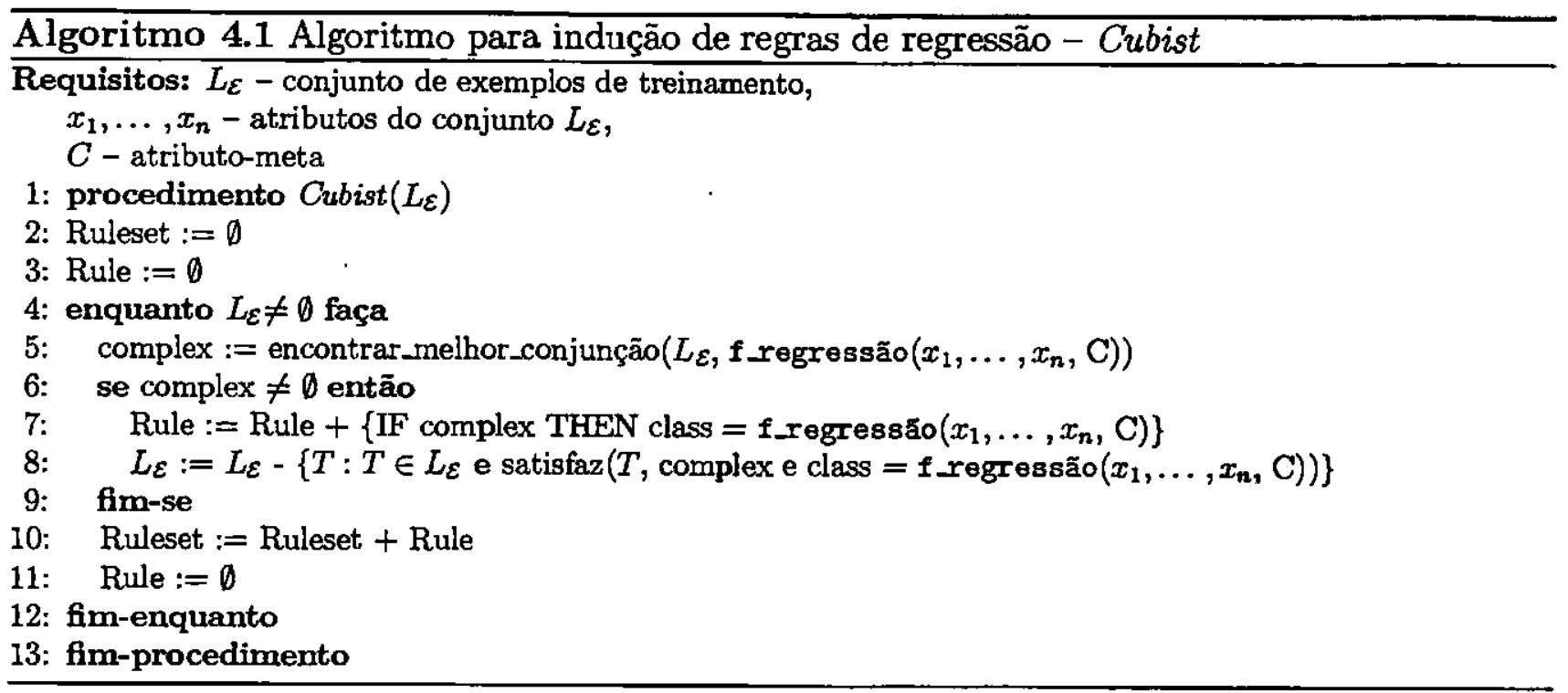

As regras geradas pelo Cubist possuem a seguinte estrutura:

$$
\begin{aligned}
<R>: & {\left[N_{\text {cob }}, V_{m}, I, E_{\text {est }}\right] } \\
& \text { if complex } \\
& \text { then }<\text { class }>=C
\end{aligned}
$$

na qual, $R$ representa o número da regra, $N_{c a b}$ é o número de exemplos cobertos pela regra, $V_{m}$ é o valor médio do atributo-meta (predito) dos exemplos cobertos, $I=\left[v_{\min }, v_{\max }\right]$ representa um intervalo de valores preditos (valor mínimo e máximo) para o atributo-meta class, $E_{\text {est }}$ é 
a estimativa de erro esperada, complex são as condições da regra $R$, e $C$ é o modelo linear (função matemática) para o atributo-meta.

Essas regras estão dispostas em ordem crescente do valor médio dos exemplos preditos $\left(V_{m}\right)$. Na Figura 4.1 podem ser observadas algumas regras obtidas no Cubist. A primeira regra possui $N_{c o b}=152, V_{m}=17.08, I=[9,38], E_{\text {est }}=1.66$, complex $=$ cylinders $>5$ e C $=-2.19-$ 0.00419 weight +0.46 model year, para 0 atributo-meta miles per gallon.

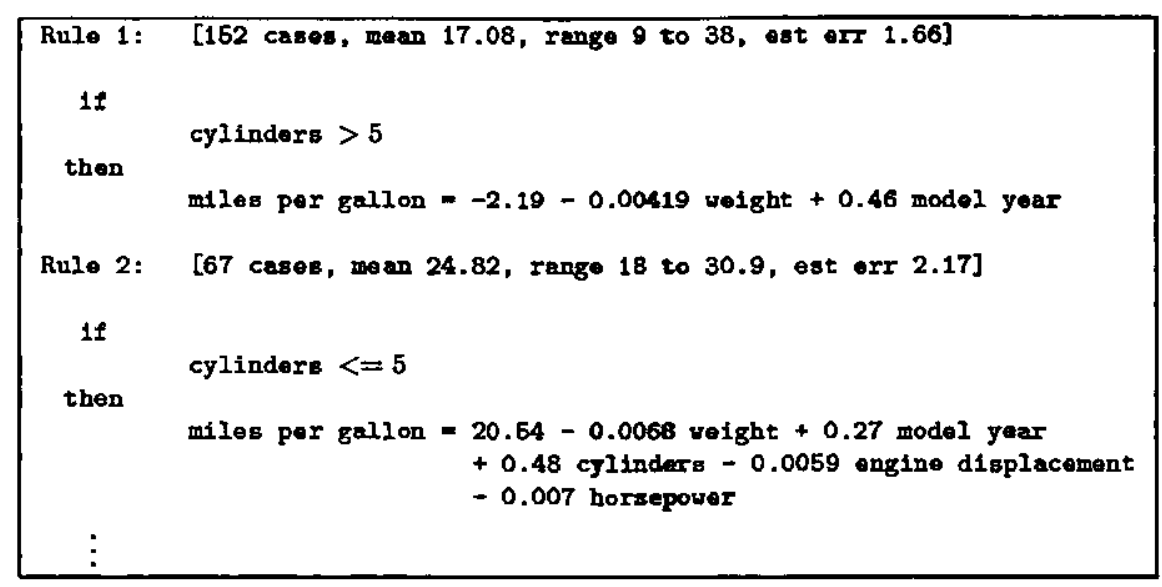

Figura 4.1: Exemplo de regras geradas pelo Cubist.

Uma regra $R_{i}$ indica que, se um caso satisfaz todas as condições, o modelo linear predito pelo Cubist é apropriado para prever o valor do atributo-meta. Se duas ou mais regras cobrem um mesmo exemplo, o Cubist faz uma média dos valores preditos para determinar um valor único para o atributo-meta.

E por fim, o Cubist avalia as regras geradas de acordo com os critérios de:

- Erro da Média: determina a magnitude de erro relacionado à média total entre os atributos;

- Coeficiente de Correlação: mede a correspondência entre os valores atuais do atributometa e os valores preditos pelo modelo linear;

- Proporção da Variação Explicada: é a medida de qualquer correspondência comum, isto é, um valor dentro de um intervalo de 0 (se o modelo linear sempre prediz o valor médio do atributo-meta) a 1 (se as previsōes do modelo linear são absolutamente corretas).

O Cubist utiliza um método híbrido que combina as regras geradas com aprendizado baseado em instâncias (Instance based) podendo obter um modelo com uma melhor precisão ao predizer 
o atributo-meta [Rulequest-Research, 2000]. Esse método funciona da seguinte forma: suponha que $X$ é o atributo-meta cujo valor deve ser predito, e $N$ é o atributo-meta de um dos vizinhos mais próximos de $X$ no conjunto de dados. O atributo-meta $N$ é conhecido e denominado de $T(N)$. Diante do fato de que o modelo baseado em regras pode ser usado para prever os atributos classe para quaisquer casos, é possível obter as previsões de $X$ e $N$ que são identificadas como $M(X)$ e $M(N)$, respectivamente. $O$ modelo então prevê que a diferença entre os valores de $X$ e $N$ é $M(X)-M(N)$ e assim, o valor de $X$ previsto pelo vizinho $N$ é ajustado para refletir esta diferença. Deste modo, Cubist utiliza $T(N)+M(X)-M(N)$ ao invés de utilizar o valor original de $N$.

\subsection{2 $\mathcal{M} 5$}

O $\mathcal{M} 5$ ou Model Tree é um algoritmo desenvolvido por Quinlan [Quinlan, 1992] para tratar variáveis (atributos) e classes contínuas. Estruturalmente, $\mathcal{M} 5$ pertence à familia TDIDT (Top Down Induction Decision Trees), mas com funções de regressão linear nos nós-folhas, ao invés de um valor categórico predizendo a classe.

O algoritmo $\mathcal{M} 5$ foi baseado no trabalho inicial de Breiman com o algoritmo CART (Classification And Regression Trees) [Breiman et al., 1984] que determina um valor numérico como classe ao invés de uma função de regressão linear. $\mathcal{M} 5$ possui algumas vantagens frente a outras técnicas que manipulam atributos contínuos, por exemplo, a Regressão Linear clássica que impõe uma relação estritamente linear entre os dados.

O $\mathcal{M} 5$ possui uma representação clara e funções de Regressão Linear que, geralmente envolvem poucos atributos [Frank et al., 1998]. O algoritmo de indução para $\mathcal{M} 5$ (Algoritmo 4.2) é similar a outros algoritmos TDIDT, isto é, existe uma fase de particionamento (Algoritmo 4.3) que divide o conjunto de dados, bem como uma fase de poda para reduzir o número de nós da árvore (Algoritmo 4.4). Além dessas duas fases, $\mathcal{M} 5$ possui uma fase adicional denominada de smoothing que reduz a discrepância de valores preditos entre os nós-folhas [Quinlan, 1992].

Cada uma dessas fases é descrita detalhadamente a seguir:

\section{Particionamento}

O processo de indução inicia com a escolha do atributo inicial que irá particionar o conjunto de dados. $O$ critério de particionamento do $\mathcal{M} 5$ faz com que o atributo escolhido maximize a redução de erro esperado, isto é, que o atributo ofereça a maior probabilidade de ter um desvio padrão mínimo. Essa função de redução de erro esperado SDR (Standard 


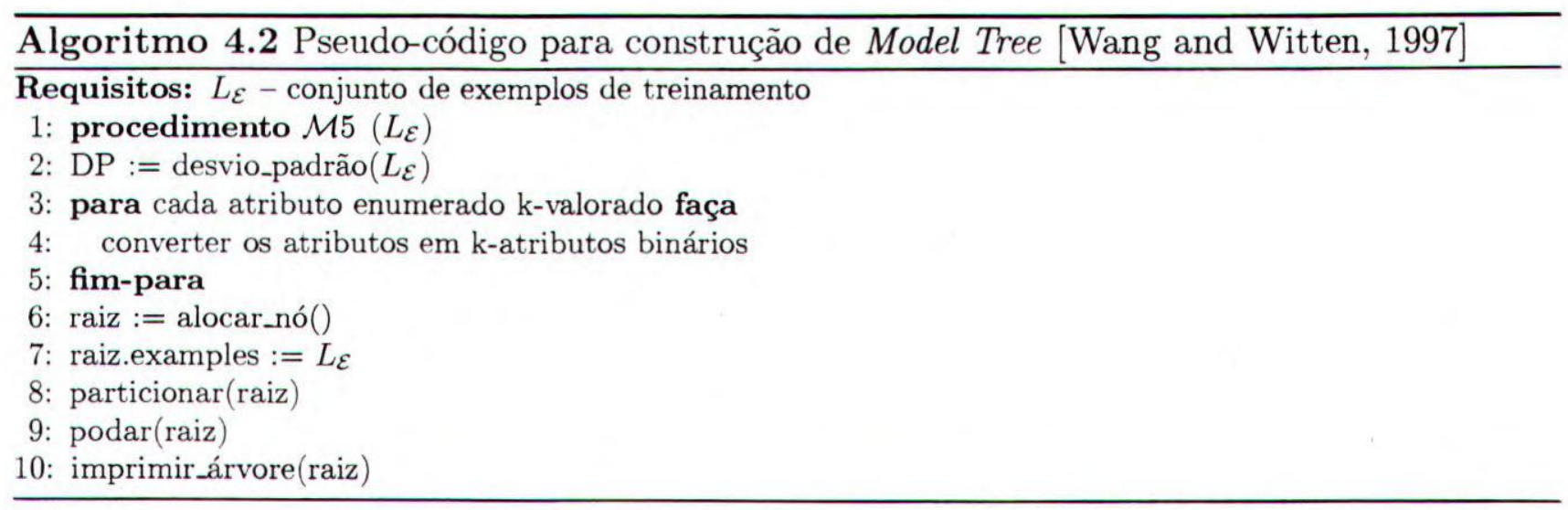

Deviation Reduction) pode ser observada na Equação 4.1 [Quinlan, 1992]:

$$
\mathrm{SDR}=d p(T)-\sum_{i \in\{D, E\}} \frac{\left|T_{i}\right|}{T} \times d p\left(T_{i}\right)
$$

na qual, $T$ é o conjunto de exemplos cobertos pelo nó de uma árvore com o atributo escolhido; $T_{D}$ e $T_{E}$ são os exemplos que resultam do particionamento e $d p$ é o desvio padrão dos valores do atributo. $\mathrm{O}$ processo de indução cessa quando restam poucas instâncias ou quando os erros de todos os exemplos cobertos pelos nós são insignificantes.

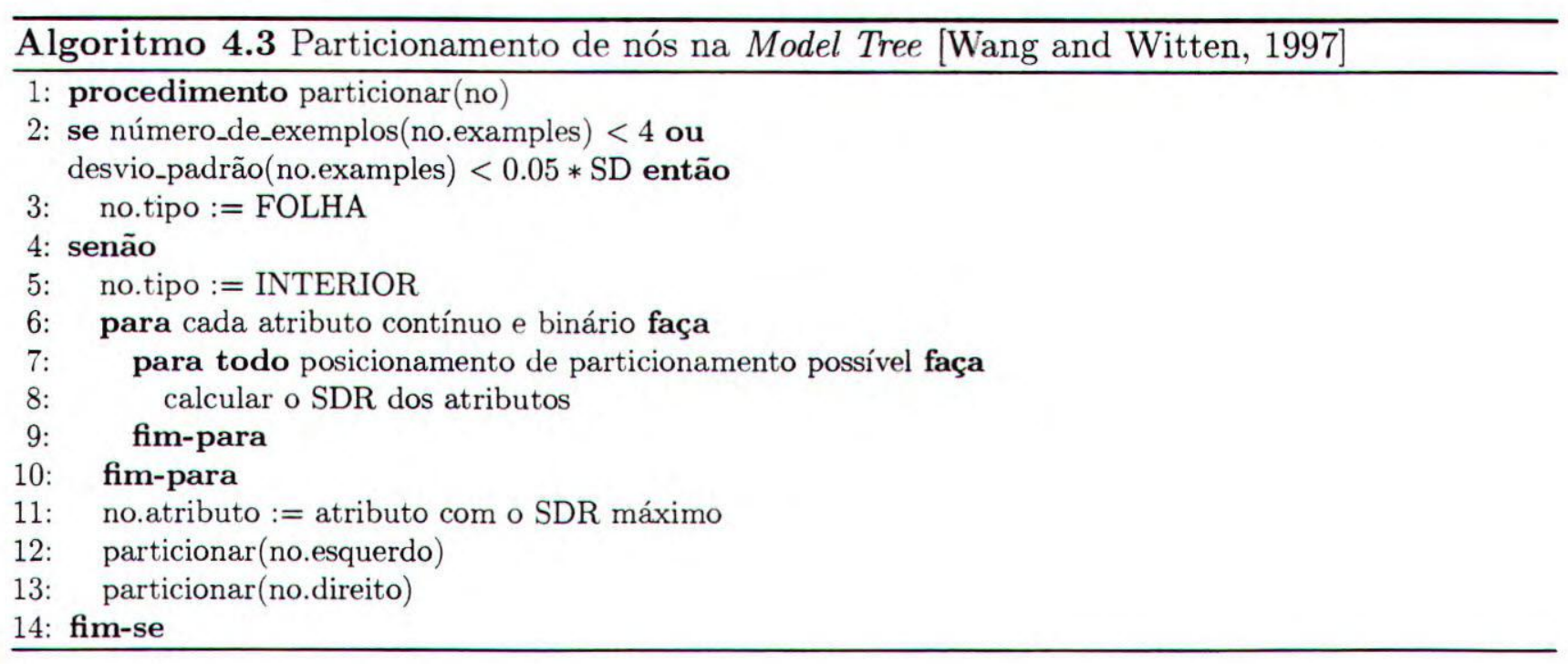




\section{Pruning}

O procedimento de pruning em $\mathcal{M} 5$ é no sentido bottom-up, isto é, a partir do nó folha até atingir o nó raiz da árvore. Este procedimento tem se tornado um padrão na poda de nós de uma árvore de decisão [Quinlan, 1986, Breiman et al., 1984], apesar que se difere dos demais, pois na retirada de um nó interior $p$, o nó é substituído por uma função de regressão linear ao invés de um valor constante, e os atributos que participam da função são aqueles que eram subordinados ao nó $p$. O procedimento de pode utiliza a medida denominada de MAD (Mean Absolute Difference), que é calculada em cada nó, segundo a Equação 3.4 na página 25. Essa estimativa, juntamente com um fator de compensação, determina a eliminação de um nó da árvore de regressão. A função de pruning pode ser observado na Equação 4.2 [Wang and Witten, 1997, Quinlan, 1992]:

$$
\operatorname{podar}(\mathrm{X})=\frac{(n+v)}{(n-v)} \times \mathrm{MAD}
$$

na qual, $n$ é o número de exemplos que chegam ao nó $\mathrm{X}$ da árvore e $v$ é o número de parâmetros da regressão linear (número de termos) que representa a classe do nó. Essa função é utilizada no Algoritmo 4.4 que retira um nó $\mathrm{X}$ da árvore de regressão se o erro estimado para $\mathrm{X}$ for menor que o erro estimado para sua subárvore.

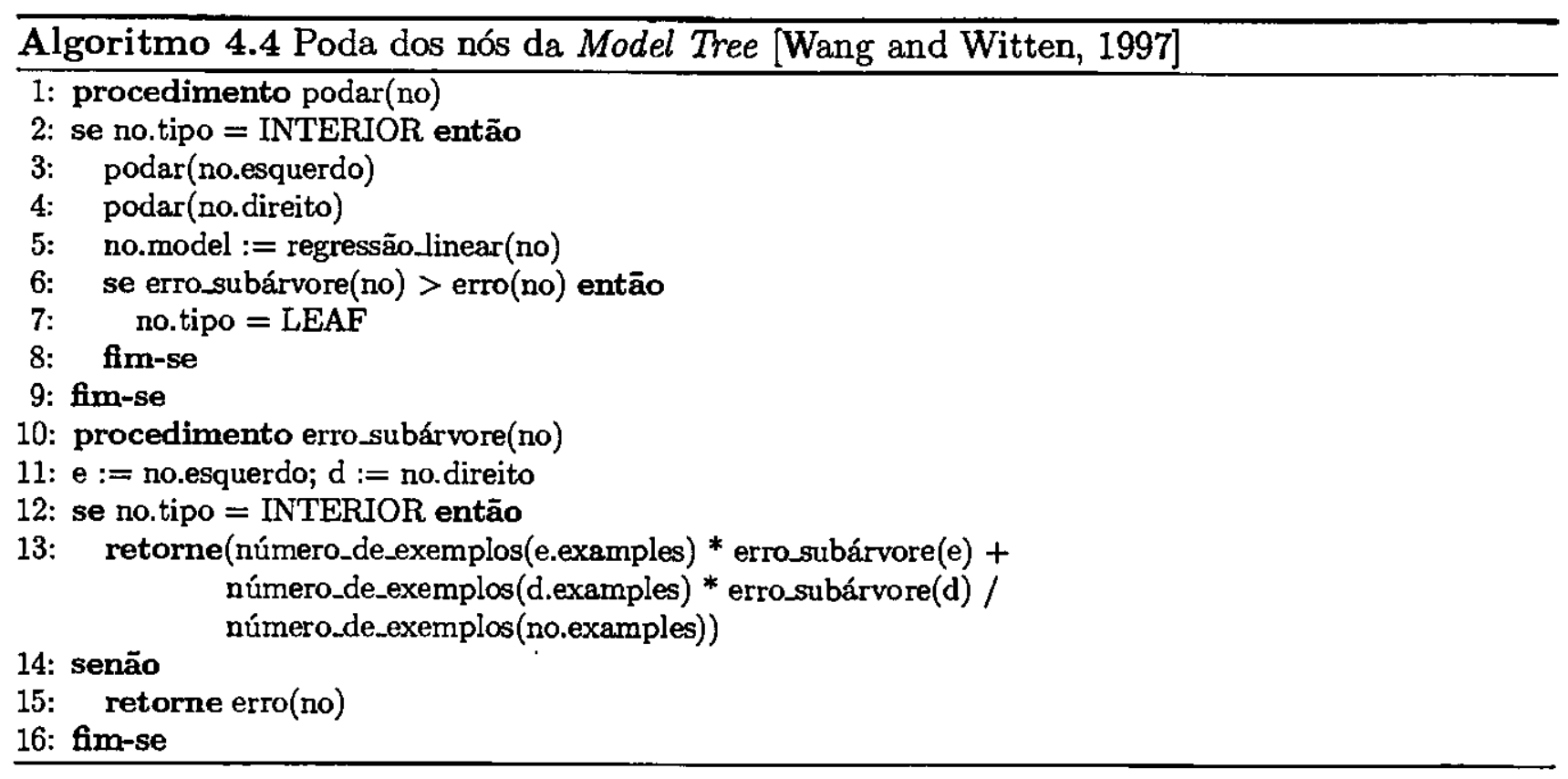




\section{Smoothing}

O processo de uniformização (smoothing) compensa as descontinuidades, inevitáveis, entre os modelos lineares adjacentes nos nós folhas da árvore podada, particularmente para alguns modelos construídos a partir de um pequeno conjunto de treinamento. Dessa forma, esse processo melhora consideravelmente a precisão e simplifica a árvore de regressão [Uysal and Güvenir, 1999, Frank et al., 1998].

Nos experimentos realizados foi utilizado um algoritmo modificado do $\mathcal{M} 5$, o $\mathcal{M} 5$ Prime, implementado na biblioteca WEKA que possui os três estágios descritos acima, com adição de estágios complementares para o tratamento de valores ausentes (missing values e valores categóricos. Um detalhamento do algoritmo $\mathcal{M} 5$ Prime pode ser encontrado em [Frank et al., 1998]. Nos experimentos realizados o algoritmo $\mathcal{M} 5$ Prime e $\mathcal{M} 5$ são considerados como sinônimos.

Além de utilizar $\mathcal{M} 5$ Prime para gerar Model Trees, pode-se utilizá-lo para gerar Regression Trees, isto é, árvores de decisão que possuem nos nós folhas valores reais preditos a partir das funçōes lineares da Model Tree gerada.

\subsection{Considerações Finais}

A utilização de ferramentas como MineSet ${ }^{T M}$ no processo Data Mining Preditivo pode auxiliar na avaliação do conhecimento extraído, devido à grande capacidade de visualização dos dados [Rezende et al., 1998] e a utilização de algoritmos de aprendizado, deve-se principalmente aos padrões encontrados no conjunto de exemplos.

Dependendo do tamanho do conjunto de exemplos são encontrados uma grande quantidade de padrões (por ex., mais de 100 regras), tornando o processo de avaliação do conhecimento uma atividade custosa. Dessa forma, torna-se necessário um ambiente de avaliação capaz de manipular essa quantidade de regras e retornar ao especialista/usuário do domínio, as regras consideradas mais "interessantes".

No próximo capítulo é descrito um ambiente de avaliação denominado de $\mathcal{R} \mathcal{R}_{\text {Evaluation }}$ que dado um conjunto de regras as avalia de acordo com as medidas comentadas no capítulo anterior. 


\section{Capítulo 5}

\section{Ambiente de Avaliação de Regras de Regressão}

\subsection{Considerações Iniciais}

O conhecimento obtido no processo Data Mining pode ser avaliado de diferentes maneiras. Uma delas é avaliar a precisão dos resultados obtidos, principalmente, quando se trata de problemas de regressão. Outras formas de avaliação do conhecimento como compreensibilidade e interessabilidade podem ser utilizadas para analisar o conhecimento extraído, apesar que avaliar os problemas de regressão utilizando essas formas de avaliação é um desafio para Data Mining.

Dentro desse contexto, neste trabalho, foram descritas algumas medidas de avaliação (Capítulo 3) para problemas de regressão, de modo a auxiliar os usuários do processo a analisar o conhecimento obtido a partir desses problemas.

Com o objetivo de apoiar usuários na análise e avaliação do conhecimento extraído de problemas de regressão é proposto um ambiente computacional denominado de $\mathcal{R R}_{\text {Evaluation }}$ Regression Rule Evaluation que é constituído por módulos inter-relacionados para obter diversas informações.

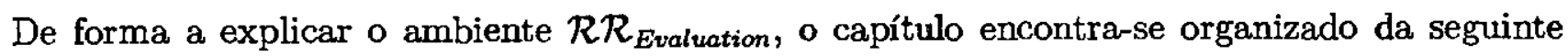
forma: na Seção 5.2 é descrita a arquitetura do Ambiente de Avaliação $\mathcal{R}_{\mathcal{R}_{\text {Evaluation, bem }}}$ como a sua funcionalidade dentro do processo de Data Mining. Na Seção 5.3 são descritos os formatos dos dados de entrada para a utilização do ambiente proposto. Na Seção 5.4 é descrita a 
ferramenta ou plug-in do ambiente, responsável pela conversão entre a representação de árvore de regressão para regras de regressão. Na Seção 5.5 é descrito módulo de regras que faz a interface entre o a base de regras e o módulo de avaliação. Na Seção 5.6 é descrito o módulo principal do ambiente, que é o de avaliação do conhecimento em problemas de regressão. Por fim, na Seção 5.7 são feitas as considerações finais do capítulo.

\subsection{Visão Geral do Ambiente $\mathcal{R} \mathcal{R}_{\text {Evaluation }}$}

O $\mathcal{R R}_{\text {Evaluation }}$ foi planejado como uma arquitetura constituída de módulos funcionais dentro do processo Data Mining. A arquitetura e implementação do $\mathcal{R} \mathcal{R}_{\text {Evaluation }}$ é aberta de forma a permitir que novas características e funcionalidades possam ser incorporadas futuramente.

O diagrama da Figura 5.1 proporciona uma visão geral de onde se insere o ambiente $\mathcal{R} \mathcal{R}_{\text {Evaluation }}$ para a avaliação do conhecimento. Pode ser observado que os padrões extraídos dos dados de treinamento são fornecidos ao ambiente $\mathcal{R R}_{\text {Evaluation }}$ para avaliação. Mas para que o ambiente possa ser utilizado é necessário que os padrōes encontrados estejam em um formato padrão. Neste trabalho, o formato padrão considerado são as regras de regressão representados na base de regras do ambiente denominada de $\mathrm{B}_{R R}$ (Base of Regression Rules).

Para a especificação do formato $\mathrm{B}_{R R}$ é necessário um módulo de conversão dos padrōes encontrados pelos algoritmos $\mathcal{M} 5$ e Cubist. No trabalho, foi desenvolvido um módulo de conversão denominado de $\mathcal{M} 5_{2}$ Rules. $\mathrm{O} \mathcal{M} 5_{2}$ Rules traduz as árvores de regressão geradas pelo $\mathcal{M} 5$ para o formato das regras de regressão da $\mathrm{B}_{R R}$. Para o Cubist não foi necessário módulo de conversão, pois a sintaxe de suas regras é condizente com a utilizada no ambiente. Outros módulos de conversão podem ser desenvolvidos para outros algoritmos de aprendizado, tendo-se a devida atenção para que esses módulos devam ter como saída as regras de regressão no formato da $\mathrm{B}_{R R}$.

Com as regras de regressão no formato da $\mathrm{B}_{R R}$, as mesmas são utilizadas pelo módulo Rules no conjunto de dados de teste ${ }^{1}$ fornecido pelo especialista para predizer o atributo-meta.

Com as predições obtidas pela utilização das regras de regressão no conjunto de teste, pode-se avaliar as regras e os valores obtidos utilizando o módulo EvaluateRules. O EvaluateRules utiliza as medidas de avaliação do conhecimento extraído, as regras da base $\mathrm{B}_{R R}$ e os valores preditos para determinar as regras, que segundo essas informações, seriam as de maior interesse

\footnotetext{
${ }^{1}$ Os dados de teste podem ser uma instância do conjunto de dados de treinamento ou um conjunto diferenciado.
} 


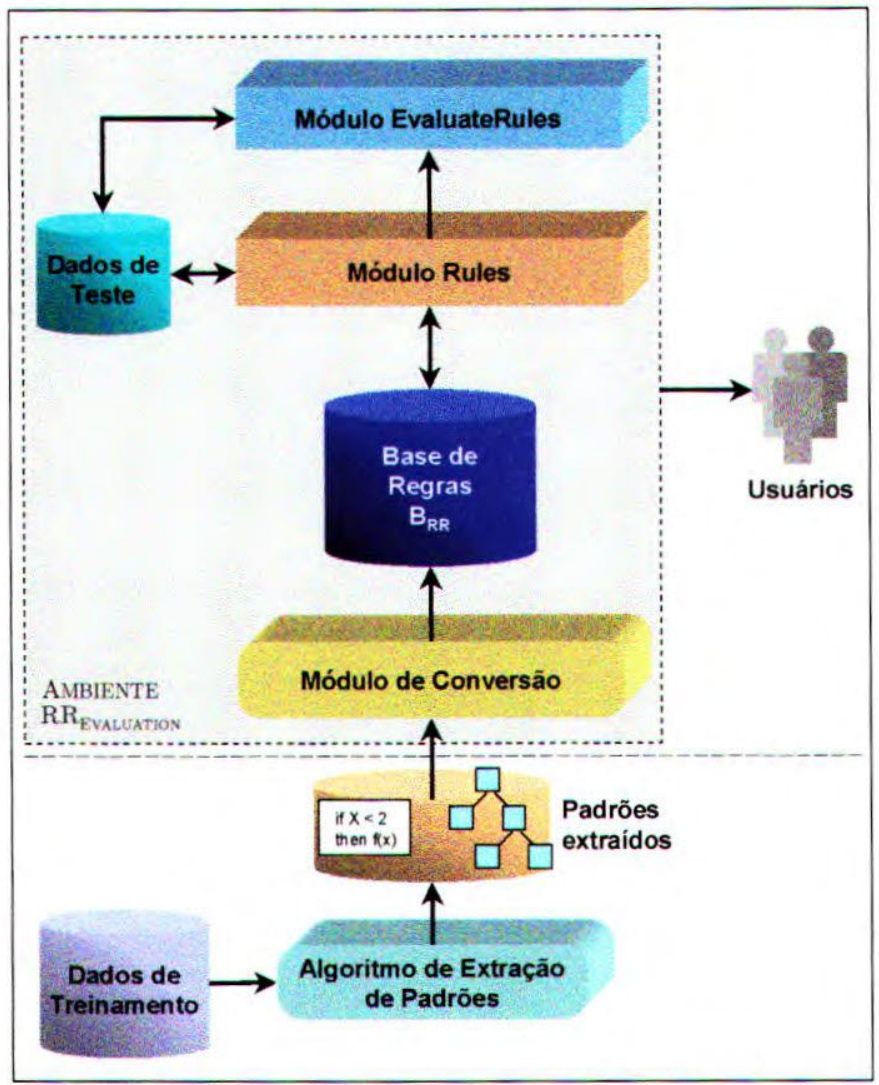

Figura 5.1: Arquitetura do Ambiente $\mathcal{R} \mathcal{R}_{\text {Evaluation. }}$

ao usuário. Além disso, esse módulo apresenta algumas opções de visualização dos resultados obtidos.

\subsection{Descrição dos Dados de Entrada para o $\mathcal{R} \mathcal{R}_{\text {Evaluation }}$}

Os dados de entrada consistem de informações para o ambiente $\mathcal{R}_{\mathcal{R}}$ Evaluation, ou seja, informações referentes as regras de regressão contidas na $\mathrm{B}_{R R}$ denominado aqui de arquivo de regras e do conjunto de dados de teste representado como o arquivo de nomes e o de dados.

Um conjunto de dados de treinamento no formato atributo-valor, retirado de [Quinlan, 1993b] e mostrado na Tabela 5.1 será utilizado para exemplificar os três arquivos de entrada do ambiente $\mathcal{R} \mathcal{R}_{\text {Evaluation }}$. 


\begin{tabular}{ccccccccc}
\hline \hline cylinders & engine & horsepower & weight & time & modelyear & origin & wagon & mpg \\
\hline 4 & 97 & 75 & 2155 & 16.4 & 76 & Japan & no & 28.0 \\
4 & 151 & 90 & 3003 & 20.1 & 80 & US & no & 24.3 \\
8 & 302 & 137 & 4042 & 14.5 & 73 & US & no & 14.0 \\
4 & 98 & 63 & 2051 & 17 & 77 & US & no & 30.5 \\
4 & 91 & 67 & 1965 & 15 & 82 & Japan & no & 38.0 \\
4 & 90 & 75 & 2125 & 14.5 & 74 & US & no & 28.0 \\
4 & 151 & 84 & 2635 & 16.4 & 81 & US & no & 26.6 \\
8 & 318 & 140 & 4080 & 13.7 & 78 & US & no & 17.5 \\
$\vdots$ & & & & & & & & \\
8 & 400 & 170 & 4746 & 12 & 71 & US & yes & 13.0 \\
8 & 351 & 149 & 4335 & 14.5 & 77 & US & no & 16.0 \\
4 & 121 & 112 & 2933 & 14.5 & 72 & Europe & yes & 18.0 \\
8 & 351 & 152 & 4215 & 12.8 & 76 & US & no & 14.5 \\
8 & 304 & 150 & 3433 & 12 & 70 & US & no & 16.0 \\
\hline \hline
\end{tabular}

Tabela 5.1: Exemplo de um conjunto de dados de treinamento.

\subsubsection{Arquivo de Nomes}

$\mathrm{O}$ arquivo de nomes contém a descrição do nome e o tipo de cada atributo do conjunto de dados. Esse arquivo segue a linguagem de representação cuja sintaxe é descrita utilizando a gramática BNF mostrada na Tabela 5.2. Ressalta-se que o atributo-meta do conjunto de dados deve ser o último atributo a ser descrito e que os demais atributos podem assumir os tipos: continuous (e.g. 10, 5.89) ou label (e.g. good, bad). Um exemplo do arquivo de nomes

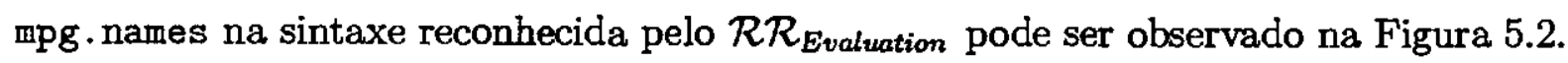

\begin{tabular}{lll|}
\hline <nomes $>$ & $:=$ & $\{\text { nome_atributo> }:<\text { tipo_atributo }>.\}^{+}$ \\
<nome_atributo> & $::=$ & a..zA..Z_ $\mid\{$a..zA..Z_0..9 \\
<tipo_atributo & $::=$ & continuous $\mid$Iabel \\
\hline
\end{tabular}

Tabela 5.2: Gramática BNF que representa a sintaxe do arquivo de nomes.

$\begin{array}{ll}\text { cylinders: continuous. } \\ \text { engine: } & \text { continuous. } \\ \text { horsepower: continuous. } \\ \text { weight: } & \text { continuous. } \\ \text { time: } & \text { continuous. } \\ \text { modelyear: continuous. } & \text { coll. } \\ \text { origin: } & \text { label. } \\ \text { wagon: } & \text { label. } \\ \text { mpg: } & \text { continuous. }\end{array}$

Figura 5.2: Exemplo do arquivo de nomes mpg.names. 
O tipo label é reconhecido como o tipo categórico. Neste tipo não é necessário especificar os valores categóricos (e.g. bad, good) a serem lidos do conjunto de dados.

\subsubsection{Arquivo de Dados}

$\mathrm{O}$ arquivo de dados contém os valores dos atributos na mesma ordem em que estão dispostos no arquivo de nomes. $O$ arquivo de dados também possui uma linguagem de representação cuja sintaxe é descrita utilizando a gramática mostrada na Tabela 5.3. Um exemplo de arquivos de dados mpg. data pode ser observado na Figura 5.3, sendo que esses dados estão organizados de acordo com o arquivo mpg. names mostrado na Figura 5.2.

\begin{tabular}{|lll|}
\hline$<$ dados $>$ & $:=$ & $<$ valor $>\{,<\text { valor }>\}^{+}$ \\
$<$valor $>$ & $::=$ & <identificador $>\mid<$numero $>$ \\
<identificador $>$ & $::=$ & {$[$a..zA..Z $]\{\text {a..zA..z_o... }\}^{+}$} \\
<numero $>$ & $::=$ & $\{0 . .9\}^{+}\left[.\{0 . .9\}^{+} \mid \lambda\right]$ \\
\hline
\end{tabular}

Tabela 5.3: Gramática BNF para a sintaxe do arquivo de nomes.

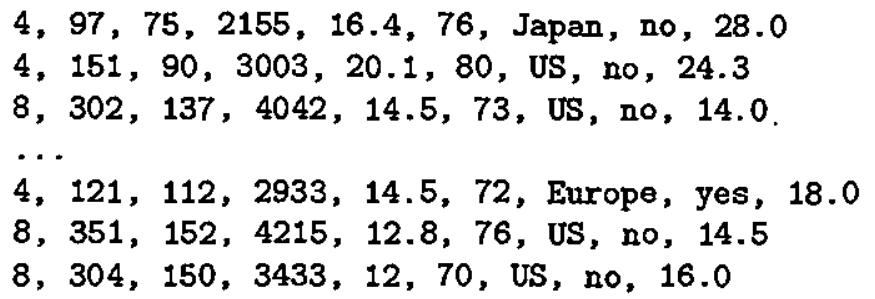

Figura 5.3: Exemplo do arquivo de dados mpg. data.

\subsubsection{Arquivo de Regras}

$\mathrm{O}$ arquivo de regras contém as regras de regressão descritas na sintaxe reconhecida pelo ambiente. A sintaxe das regras é definida pela gramática BNF mostrada na Tabela 5.4. Deve ser observado que a gramática por nós definida é muito semelhante a utilizada por alguns algoritmos de aprendizado pará a geração de regras. Um exemplo de um arquivo de regras denominado de mpg.rules reconhecido pelo ambiente pode ser observado na Figura 5.4.

As regras extraídas do Cubist são inseridas diretamente no ambiente, adicionando-se à $B_{R R}$, enquanto as árvores de regressão geradas pelo $\mathcal{M} 5$ necessitam de um módulo de conversão para que possam ser extraídas as regras no formato da $B_{R R}$. Essas regras estão dispostas de acordo com o percurso "in-order" realizado na árvore pelo $\mathcal{M} 5_{2} \mathrm{Rules,} \mathrm{a} \mathrm{ser} \mathrm{descrito} \mathrm{na} \mathrm{próxima} \mathrm{seção.}$ 


\begin{tabular}{|c|c|c|}
\hline$<$ regra $>$ & $::=$ & $\begin{array}{l}\text { if }\{<\text { condicao }>\}^{+} \\
\text {then }<\text { conclusao }>\end{array}$ \\
\hline$<$ condicao $>$ & $::=$ & $<$ identificador $><$ operador_relacional $><$ valor $>$ \\
\hline <operador_relacional > & $::=$ & $<|<=|>|>=|=\mid$ in \\
\hline$<$ valor $>$ & $::=$ & $\begin{array}{l}<\text { numero }>\mid \\
\left\{<\text { numero }>\{,<\text { numero }>\}^{*}\right\} \mid \\
<\text { identificador }>\end{array}$ \\
\hline$<$ conclusao $>$ & $::=$ & $<$ identificador $>=<$ expressao $>$ \\
\hline <expressao > & $::=$ & $\begin{array}{l}[-] \mid<\text { numero }>\mid<\text { identificador }>][+\mid-]<\text { termo }>1 \\
<\text { expressao }>\mid \\
|+| \rightarrow \mid<\text { numero }><\text { identificador }>\text { in }\{<\text { listaid }>\}\end{array}$ \\
\hline$<$ lista_id $>$ & $::=$ & $<$ identificador $>\{,<$ identificador $>\}$ \\
\hline$<$ termo $>$ & $::=$ & $<$ numero $><$ expressao $>$ \\
\hline$<$ numero $>$ & $::=$ & $\{0 . .9\}^{+}\left[.\{0 . .9\}^{+} \mid \lambda\right]$ \\
\hline$<$ identificador $>$ & $::=$ & {$\left[a . . z A . . Z_{. .}\right]\left\{a . . z A . . Z_{-} 0 . .9\right\}$} \\
\hline
\end{tabular}

Tabela 5.4: Gramática BNF usada para definir a sintaxe das regras da base $B_{R R}$ do ambiente $\mathcal{R} \mathcal{R}_{\text {Evaluation }}$.

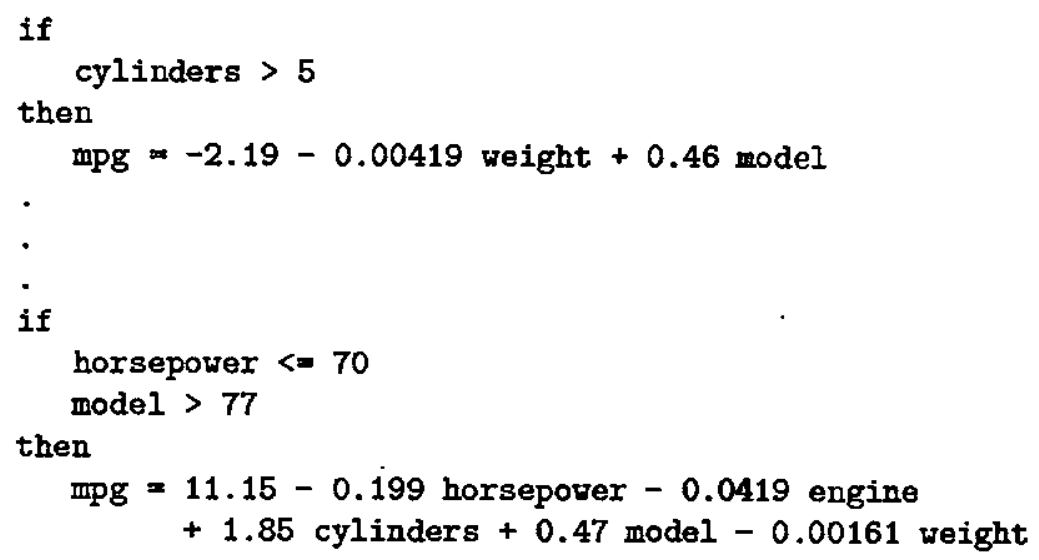

Figura 5.4: Exemplo do arquivo de regras de regressão mpg. rules.

\subsection{Módulo de Conversão de Padrões para o formato da $\mathrm{B}_{R R}$}

A existência de um módulo de conversão no ambiente faz-se pela necessidade de traduzir a representação de árvores de regiessão gerada pelo $\mathcal{M} 5$ para as regras de regressão da $\mathrm{B}_{R R}$. Com essa conversão é possível comparar os algoritmos $\mathcal{M} 5$ e Cubist quanto a predição de um atributo-meta contínuo, bem como, avaliar os padrões encontrados por ambos. $O$ módulo, existente dentro do ambiente, responsável por essa conversão é o $\mathcal{M} 5_{2}$ Rules.

$\mathrm{O} \mathcal{M} 5_{2}$ Rules consiste de um interpretador que faz a traduçāo da representação de árvores de regressão geradas pelo $\mathcal{M} 5$ para o formato de regras de regressão na sintaxe especificada pela 
gramática da Tabela 5.4 .

Para exemplificar o funcionamento do módulo, considere a representação gráfica de uma árvore de regressão gerada pelo $\mathcal{M} 5$ para o conjunto de dados da Tabela 5.1, mostrada na Figura 5.5(a), enquanto que na Figura 5.5(b) é mostrada a estrutura textual da mesma árvore. Os nós-folhas com iniciais LM - Linear Models - representados no exemplo da Figura 5.5(a e b) são os modelos lineares (e.g. funções matemáticas lineares) para predizer o atributo-meta mpg. As variáveis horsepower, weight, modelyear, time e origin representam os atributos ou preditores utilizados nas funções matemáticas das regras de regressão.

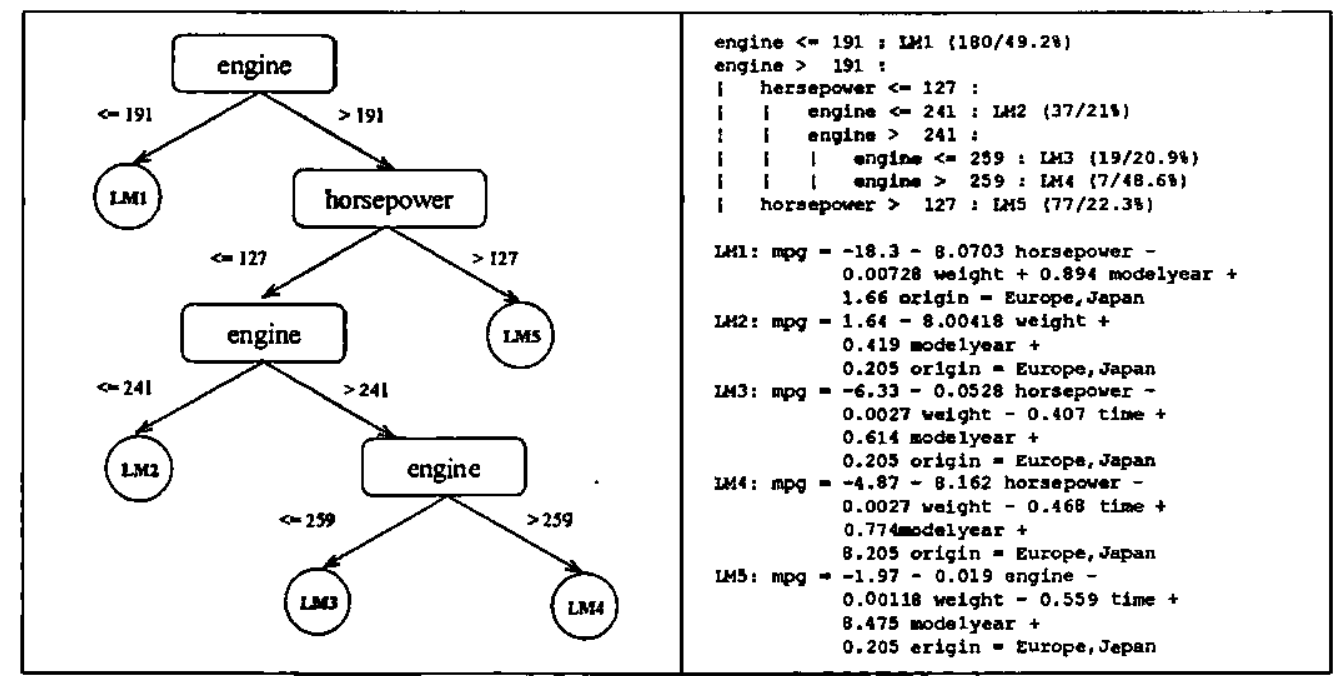

(a)

(b)

Figura 5.5: Representação gráfica da árvore de regressão (a) e as regras geradas a partir da árvore (b).

Na Figura 5.6 podem ser observadas as regras obtidas pela traduçāo da árvore de regressão para a sintaxe da base $\mathrm{B}_{R R}$.

\subsection{Módulo Rules}

O módulo Rules que foi desenvolvido consiste de um interpretador de regras de regressão da base $\mathrm{B}_{R R}$ a serem utilizadas no conjunto de dados de teste. Esse interpretador é responsável pelo armazenamento das informações referentes as regras de regressão, tais como: número de exemplos cobertos, número de condições e de preditores, média dos valores preditos e o desvio padrão. 


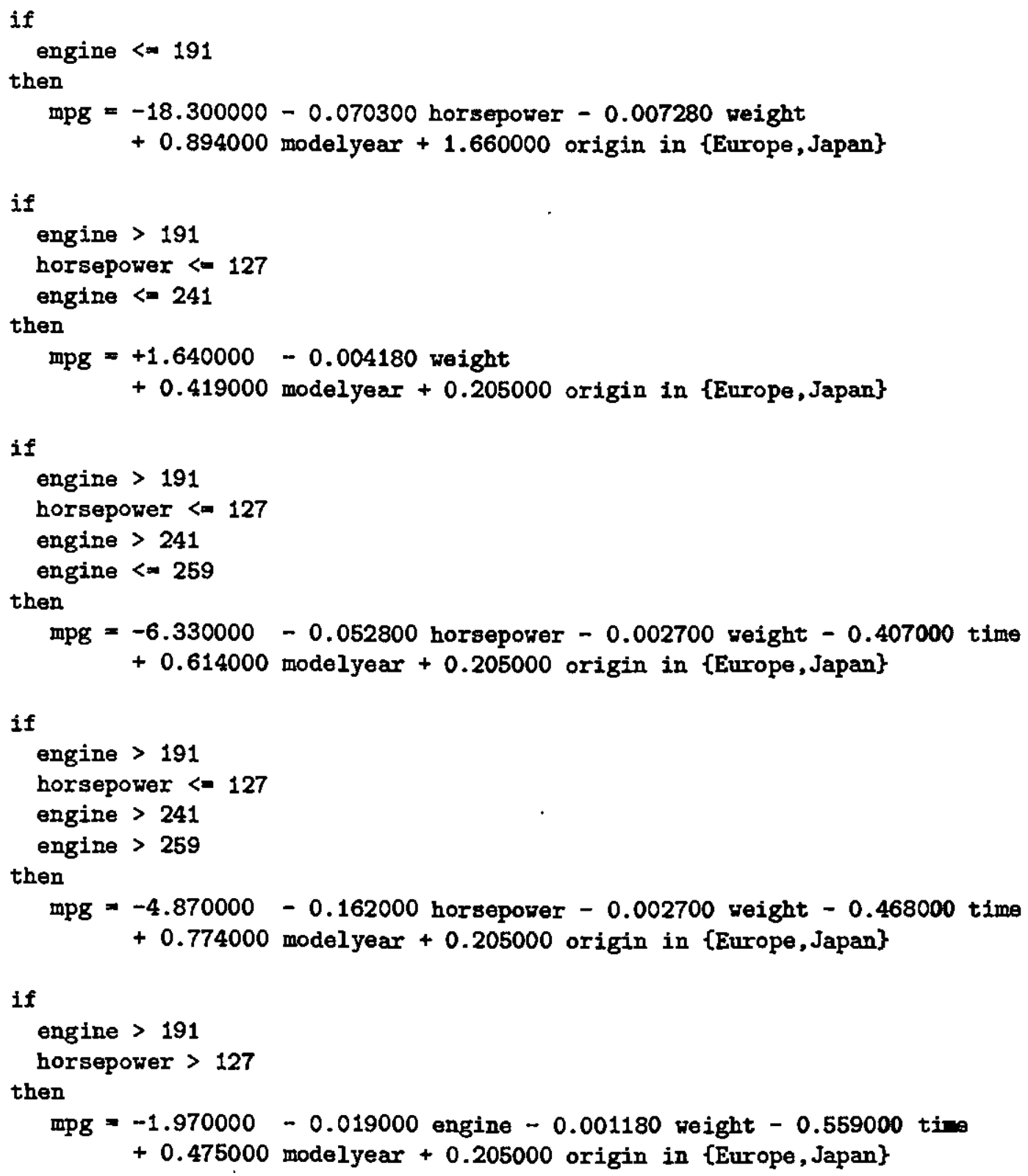

Figura 5.6: Regras de regressão traduzidas pelo $\mathcal{M} 5_{2}$ Rules.

O módulo Rules é uma interface entre a base de regras e o módulo EvaluateRules, pois através dos resultados obtidos com a utilização do Rules no conjunto de dados de teste é que o EvaluateRules calcula as medidas de avaliaçāo do conhecimento extraído. 


\subsection{Módulo de Avaliação EvaluateRules}

O módulo de avaliação de regras EvaluateRules que foi desenvolvido como parte do ambiente $\mathcal{R}_{\mathcal{R}_{\text {Evaluation }}}$ é responsável por aplicar as medidas de avaliação do conhecimento extraído, bem como mostrar os resultados ao usuário. Para isso, foi implementada uma interface dividida em 3 guias de orientação: Datasets, Graphics e Statistical Analysis. Essas guias oferecem diferentes recursos de forma a auxiliar os usuários na avaliação do conhecimento obtido.

\subsubsection{Guia Datasets}

Nesta guia podem ser mostrados os exemplos do conjunto de teste cobertos por cada regra de regressão, bem como o valor predito para o atributo-meta de cada exemplo. Na Figura 5.7 pode ser observada a interface do EvaluateRules com a guia Datasets selecionada.

\begin{tabular}{|c|c|c|c|c|c|c|c|c|c|c|}
\hline \multicolumn{11}{|c|}{ Regression Rules Quality Analysis } \\
\hline Datasets Graphics & Statistical An & nalusis | & & & & & & & & \\
\hline \multirow{3}{*}{ 5 AlExamples } & \# & CRIM & $\mathrm{ZN}$ & INDUS & CHAS & NOX & AM & AGE & DIS & RAD \\
\hline & 1 & 01285 & 25 & 5.13 & 0 & 0.453 & 6.762 & 43.4 & 7.9809 & 8 \\
\hline & 2 & 0.10469 & 40 & 6.41 & 1 & 0.447 & 7.267 & 49 & 4.7872 & 4 \\
\hline \multirow{3}{*}{5 Rulel } & 3 & 0.17446 & 0 & 10.59 & 1 & 0.489 & 5.96 & 92.1 & 3.8771 & 4 \\
\hline & 4 & 0.05372 & 0 & 13.92 & 0 & 0.437 & 6.549 & 51 & 5.9604 & 4 \\
\hline & 5 & 0.03932 & 0 & 3.41 & 0 & 0.489 & 6.405 & 73.9 & 3.0921 & 2 \\
\hline \multirow[t]{2}{*}{5 Rule2 } & 6 & 5.69175 & 0 & 18.1 & 0 & 0.583 & 6.114 & 79.8 & 3.5459 & 24 \\
\hline & 7 & 0.0795 & 60 & 1.69 & 0 & 0.411 & 6.579 & 35.9 & 10.7103 & 4 \\
\hline \multirow[t]{2}{*}{ C Rule3 } & 8 & 1.12658 & 0 & 19.58 & 1 & 0.871 & 5.012 & 88 & 1.6102 & 5 \\
\hline & 9 & 0.0459 & 52.5 & 5.32 & 0 & 0.405 & 6.315 & 45.6 & 7.3172 & 6 \\
\hline \multirow{3}{*}{ C Rule4 } & 10 & 0.00632 & 18 & 2.31 & 0 & 0.538 & 6.575 & 65.2 & 4.09 & 1 \\
\hline & 11 & 73.5341 & 0 & 18.1 & 0 & 0.679 & 5.957 & 100 & 1.8026 & 24 \\
\hline & 12 & 3.47428 & 0 & 18.1 & 1 & 0.718 & 8.78 & 82.9 & 1.9047 & 24 \\
\hline \multirow{2}{*}{ C Rule5 } & 13 & 37.6619 & 0 & 18.1 & 0 & 0.679 & 6.202 & 78.7 & 1.8629 & 24 \\
\hline & 14 & 0.1403 & 22 & 5.86 & 0 & 0.431 & 6.487 & 13 & 7.3967 & 7 \\
\hline \multirow[t]{2}{*}{ C Rule6 } & 15 & 0.44791 & 0 & 6.2 & 1 & 0.507 & 6.726 & 66.5 & 3.6519 & 8 \\
\hline & 16 & 0.02731 & 0 & 7.07 & 0 & 0.469 & 6.421 & 78.9 & 4.9671 & 2 \\
\hline \multirow{3}{*}{ C Rule? } & 17 & 0.09164 & 0 & 10.81 & 0 & 0.413 & 6.065 & 7.8 & 5.2873 & 4 \\
\hline & 18 & 15.8744 & 0 & 18.1 & 0 & 0.671 & 6.545 & 99.1 & 1.5192 & 24 \\
\hline & 19 & 0.43571 & 0 & 10.59 & 1 & 0.489 & 5.344 & 100 & 3.875 & 4 \\
\hline \multirow[t]{2}{*}{ C RuleB } & 20 & 0.0536 & 21 & 5.64 & 0 & 0.439 & 6.511 & 21.1 & 6.8147 & 4 \\
\hline & 21 & 0.09068 & 45 & 3.44 & 0 & 0.437 & 6.951 & 21.5 & 6.4798 & 5 \\
\hline \multirow[t]{2}{*}{ C Ruleg } & 22 & 17.8667 & 0 & 18.1 & 0 & 0.671 & 6.223 & 100 & 1.3861 & 24 \\
\hline & 23 & 3.69695 & 0 & 18.1 & 0 & 0.718 & 4.963 & 91.4 & 1.7523 & 24 \\
\hline \multirow[t]{2}{*}{ C Rule10 } & 24 & 0.05735 & 0 & 4.49 & 0 & 0.449 & 6.63 & 56.1 & 4.4377 & 3 \\
\hline & +1) & amen & n & $\infty$ & n & $n$ & n... & $\cdots$ & arame & 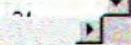 \\
\hline
\end{tabular}

Figura 5.7: Guia de observação dos dados no Módulo EvaluateRules do $\mathcal{R} \mathcal{R}_{\text {Evaluation }}$.

Além disso, também pode ser selecionada uma regra de regressão em particular e o módulo EvaluateRules mostra os exemplos cobertos por esta regra. Na Figura 5.8 pode ser observada 
a seleção da regra 6 da $\mathrm{B}_{R R}$, mostrando os exemplos cobertos e o número de exemplos cobertos pela regra.

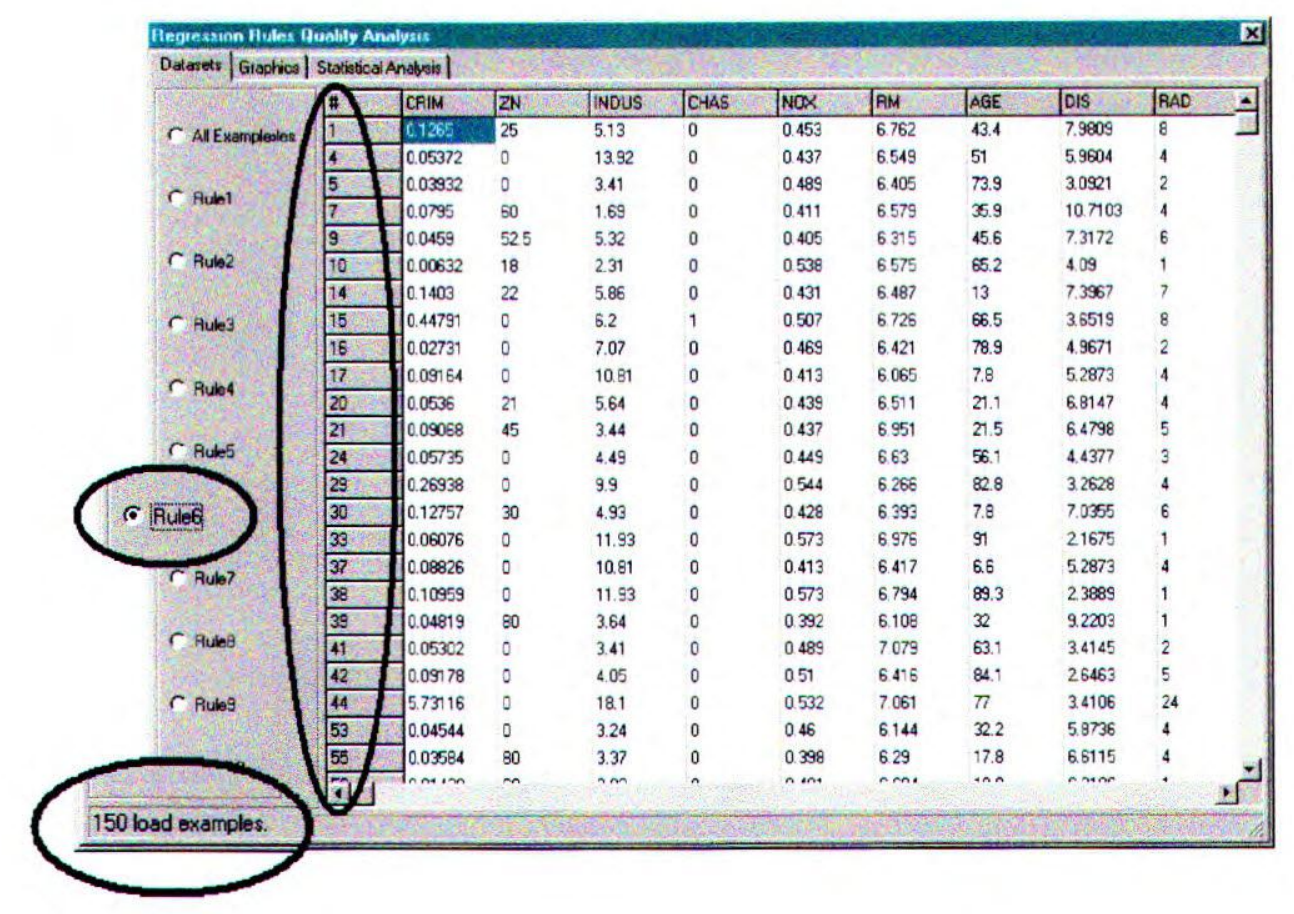

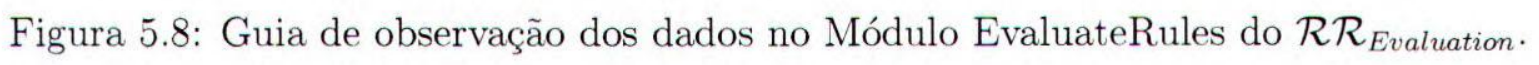

\subsubsection{Guia Graphics}

Nesta guia podem ser observados os valores reais e preditos do conjunto de exemplos de teste plotados em um gráfico 2D. No eixo X do gráfico estão os exemplos de treinamento e no eixo Y estão os valores reais e preditos para cada exemplo. A linha do gráfico destacada em vermelho são os valores reais enquanto a linha destacada em verde são os valores preditos. Na Figura 5.9 pode ser observado o gráfico plotado (Real $\times$ Predito) para todos os exemplos do conjunto de dados.

Esta guia possui uma dependência com a guia Datasets, pois de acordo com a regra selecionada, o gráfico plotado será respectivo àquela regra, com seus respectivos valores reais e preditos. Na Figura 5.10 pode ser a observado gráfico plotado para a regra 6 selecionada na Figura 5.8. Além disso, existe a opção de ordenar os valores reais do grafico, resultando no gráfico da Figura 5.11. 


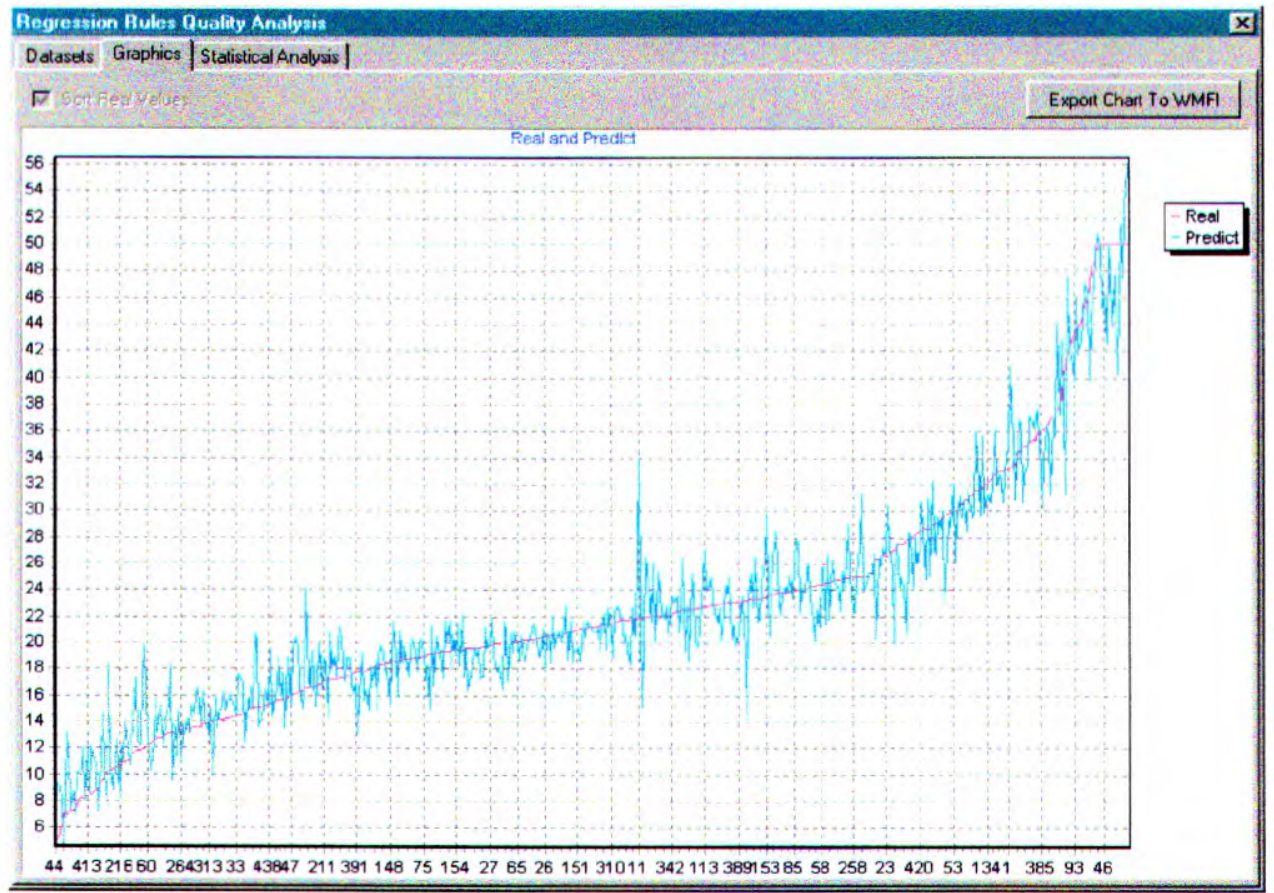

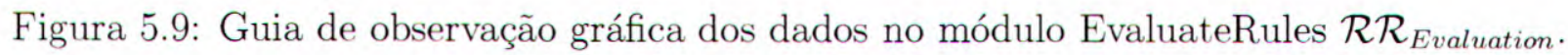

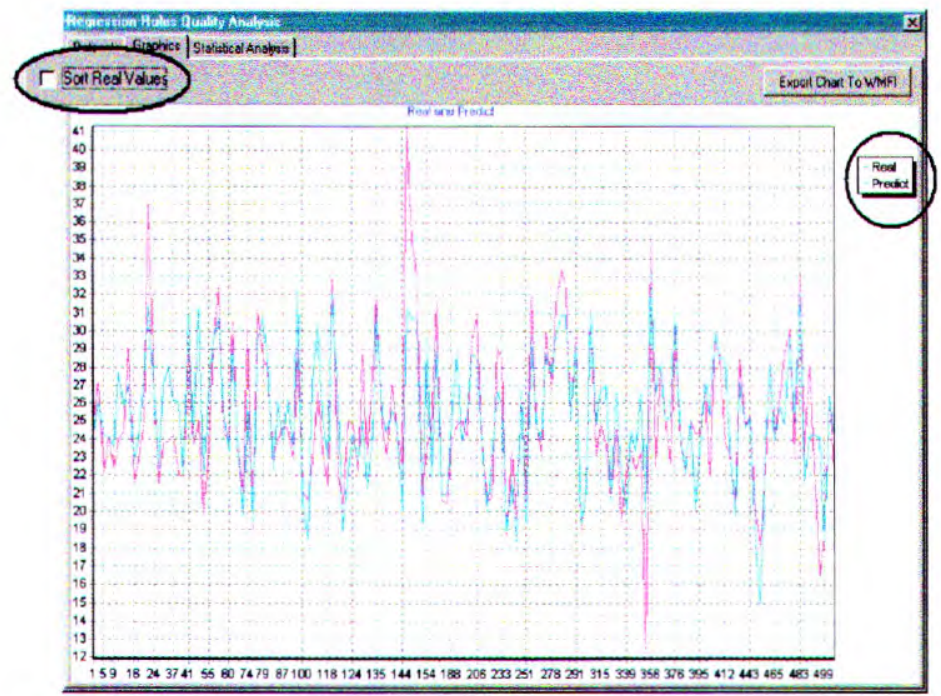

Figura 5.10: Gráfico plotado para a regra 6 selecionada.

\subsubsection{Guia Statistical Analysis}

Nesta guia são feitos os cálculos das medidas de precisão, compreensibilidade e interessabilidade das regras de regressão da $\mathrm{B}_{R R}$. Na Figura 5.12 pode ser observada a interface da guia de 


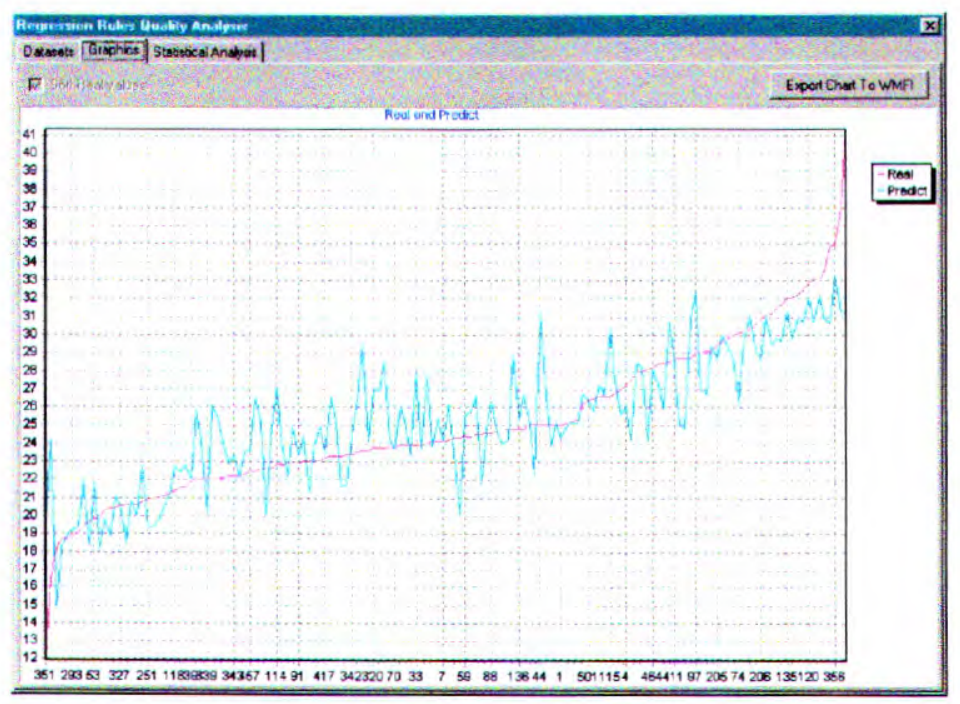

Figura 5.11: Gráfico plotado em ordem crescente do valor real do atributo-meta para a regra 6 selecionada.

análise estatística. Além do cálculo das medidas de avaliação, pode-se modificar o peso da $w_{\text {ganho }}{ }^{2}$ delimitando um intervalo ou um valor constante. Existe também a opção de ordenar as medidas e de exportar os resultados obtidos para arquivos textos. No ambiente, atualmente, os resultados obtidos podem ser exportados somente para o formato texto ou em IATEX.

\subsection{Considerações Finais}

Neste capítulo foi apresentado o ambiente computacional $\mathcal{R} \mathcal{R}_{\text {Evaluation }}$ que consiste de uma ferramenta para auxiliar os usuários de Data Mining a determinar regras de interesse, segundo os critérios de precisão, compreensibilidade e interessabilidade propostos em capítulos anteriores.

Uma descrição detalhada do ambiente $\mathcal{R}_{\mathcal{R}}$ Evaluation, bem como um exemplo de utilização do mesmo pode ser encontrado em [Nagai and Rezende, 2000].

No próximo capítulo são descritos os experimentos realizados em diversos conjuntos nos mais diferentes domínios, utilizando-se essa ferramenta, principalmente, para determinar e analisar as regras de regressão obtidas nesses domínios.

\footnotetext{
${ }^{2}$ Valor utilizado para cálculo das medidas de interessabilidade.
} 


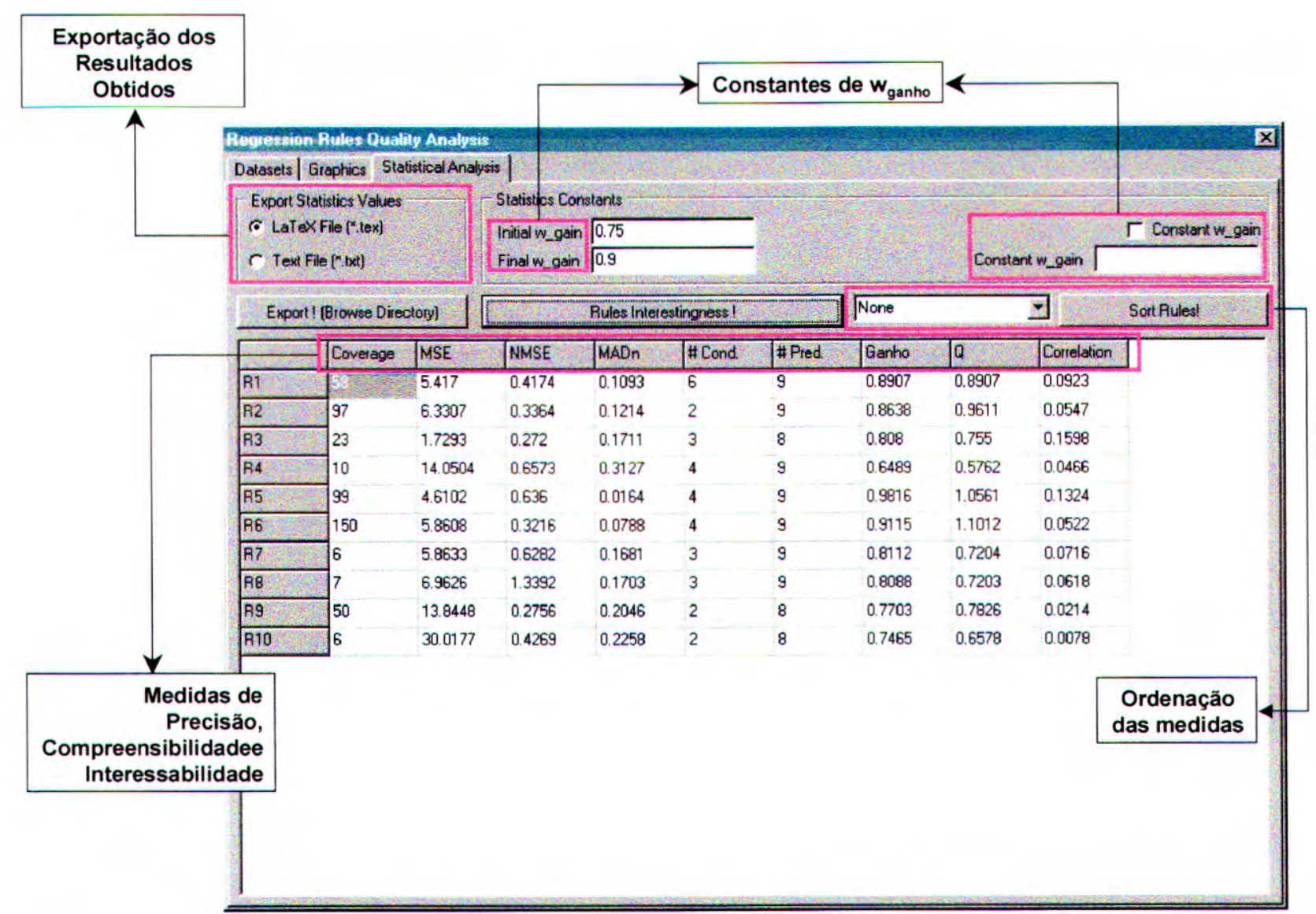

Figura 5.12: Guia das medidas de avaliação de conhecimento no módulo EvaluateRules do $\mathcal{R} \mathcal{R}_{\text {Evaluation }}$. 


\section{Capítulo 6}

\section{Experimentos Realizados}

\subsection{Considerações Iniciais}

Neste capítulo são descritos os diversos experimentos realizados em conjuntos de dados de vários domínios. Desses conjuntos de dados foram extraídos padrões utilizando-se os algoritmos $\mathcal{M} 5$ e Cubist, que posteriormente foram avaliados pelas medidas de avaliação de conhecimento de problemas de regressão descritas na Seção 3.5 na página 23, e que foram implementadas no ambiente $\mathcal{R}_{\mathcal{R}_{\text {Evaluation. }} \text {. }} \mathrm{O} \mathcal{R} \mathcal{R}_{\text {Evaluation }}$ foi utilizado para avaliar as regras de regressão, retornando aquelas de maior interesse, segundo as medidas de avaliação do conhecimento.

De modo a descrever os resultados obtidos pela avaliação do conhecimento extraído nos experimentos realizados, o capítulo encontra-se organizado da seguinte forma: na Seção 6.2 são descritos os conjuntos de dados utilizados, detalhando-se os atributos e informações pertinentes a cada um deles. Na Seção 6.3 é descrita a metodologia utilizada nos experimentos. Na Seção 6.4 são descritos os resultados obtidos ao utilizar as medidas de precisão, compreensibilidade e interessabilidade. Por último, na Seção 6.5 são feitas as considerações finais do capítulo.

\subsection{Dados Utilizados}

Os experimentos aqui descritos foram realizados com dados de 6 domínios diferentes, que são:

- Os conjuntos de dados CPU, Boston Housing e MPG retirados do Repositório Irvine UCI [Blake et al., 1998]. O primeiro contém atributos que denotam o desempenho de CPU's 
de diferentes configurações, o segundo possui atributos a respeito de imóveis da cidade de Boston e o terceiro contém atributos a respeito do consumo de gasolina de automóveis de diferentes marcas e países.

- Um conjunto de dados contendo informações sobre a situação da camada de ozônio na cidade de Los Angeles, denominado de Ozone [Breiman and Friedman, 1985];

- Um conjunto de dados contendo informações de poços petrolíferos em Big Escambia Creek - Alabama/EUA, denominado de Permeability [Rogers et al., 1995]; e

- Um subconjunto dos dados da Base de Dados do Programa de Melhoramento Genético da Raça Nelore - PMGRN [Lôbo et al., 1999], denominado de PMGRN-455.

\subsubsection{Descrição Detalhada dos Conjuntos de Dados}

Nessa subseção é feita uma breve descrição de cada conjunto de dados utilizado nos experimentos. Para cada conjunto de dados é apresentada uma tabela contendo algumas características desses conjuntos de dados, tais como: o número do atributo (ordem dentro do conjunto de dados), os nomes do atributos, número de valores distintos, valor mínimo, valor máximo, erro e tipos dos atributos. Todas as informações foram obtidas utilizando-se o Weka Knowledge Explorer do software WEKA [Witten and Frank, 1999].

O primeiro conjunto de dados a ser detalhado é o conjunto de dados CPU, seguido do Boston Housing, MPG, Ozone, Permeability e do PMGRN-455.

CPU Esse conjunto de dados foi doado por David W. Aha para o Repositório da UCI. O conjunto de dados é formado por 209 exemplos e 7 atributos contínuos, sendo que todos esses atributos são referentes a dados de desempenho de uma CPU. Esse conjunto de dados foi utilizado em um trabalho de [Kibler and Aha, 1988], que utilizou métodos baseados em instâncias para predizer o desempenho relativo da CPU. Na Tabela 6.1 pode ser observada uma descrição detalhada desse conjunto de dados.

Boston Housing Esse conjunto de dados foi doado por D. Harrison e D. L. Rubinfeld, pesquisadores da Universidade Carnegie Mellon para o Repositório da UCI e é formado por fatores sócio-econômicos que determinam a compra de imóveis em subúrbios de Boston. O conjunto de dados é formado por 506 exemplos e 14 atributos contínuos. Esse conjunto de dados foi utilizado em um trabalho de [Quinlan, 1993b] sobre a combinação de aprendizados baseados em regras e instâncias para melhorar a precisão das regras. Na 


\begin{tabular}{r|l|lrr|r|r}
\hline \hline $\begin{array}{r}\text { Número do } \\
\text { atributo }\end{array}$ & $\begin{array}{l}\text { Nome do } \\
\text { utributo }\end{array}$ & $\begin{array}{l}\text { \# Valores } \\
\text { distintos }\end{array}$ & $\begin{array}{r}\text { Vulor } \\
\text { minimo }\end{array}$ & $\begin{array}{r}\text { Vulox } \\
\text { máximo }\end{array}$ & Médis \pm DP & $\begin{array}{l}\text { Tipo do } \\
\text { Atributo }\end{array}$ \\
\hline \#1 & MYCT & 60 & 17 & 1500 & $203.82 \pm 260.26$ & contínuo \\
$\# 2$ & MMIN & 25 & 64 & 32000 & $2867.98 \pm 3878.74$ & contínuo \\
$\# 3$ & MMAX & 23 & 64 & 64000 & $11796.15 \pm 11726.56$ & contínuo \\
$\# 4$ & CACH & 22 & 0 & 256 & $25.21 \pm 40.63$ & contínuo \\
$\# 5$ & CHMIN & 15 & 0 & 52 & $4.70 \pm 6.82$ & contínuo \\
$\# 6$ & CHMAX & 31 & 0 & 176 & $18.27 \pm 26.0$ & contínuo \\
$\# 7$ & Performance & 116 & 6 & 1150 & $105.62 \pm 160.83$ & contínuo \\
\hline
\end{tabular}

Tabela 6.1: Descrição detalhada do conjunto de dados CPU.

Tabela 6.2 podem ser observadas algumas características detalhadas dos atributos desse conjunto de dados.

\begin{tabular}{|c|c|c|c|c|c|c|}
\hline $\begin{array}{r}\text { Número do } \\
\text { atributo }\end{array}$ & $\begin{array}{l}\text { Nome do } \\
\text { atributo }\end{array}$ & $\begin{array}{l}\text { \# Vulores } \\
\text { distintos }\end{array}$ & $\begin{array}{r}\text { Vulor } \\
\text { mínimo }\end{array}$ & $\begin{array}{r}\text { Valor } \\
\text { máximo }\end{array}$ & Média $\pm D P$ & $\begin{array}{l}\text { Tipo do } \\
\text { atributo }\end{array}$ \\
\hline \#1 & CRIM & 504 & $0 . \overline{0.0632}$ & 88.9762 & $3.61 \pm 8.60$ & contínuo \\
\hline \#2 & $\mathrm{ZN}$ & 26 & 0.0 & 100.0 & $11.36 \pm 23.32$ & contínuo \\
\hline \#3 & INDUS & 76 & 0.46 & 27.74 & $11.14 \pm 6.86$ & contínuo \\
\hline \#4 & CHAS & 2 & 0 & $\mathbf{1}$ & - & contínuo \\
\hline \#5 & NOX & 81 & 0.385 & 0.871 & $0.55 \pm 0.12$ & contínuo \\
\hline \#6 & RM & 446 & 3.561 & 8.78 & $6.28 \pm 0.70$ & contínuo \\
\hline \#7 & AGE & 356 & 2.9 & 100.0 & $68.57 \pm 28.15$ & contínuo \\
\hline \#8 & DIS & 412 & 1.1296 & 12.1265 & $3.79 \pm 2.10$ & contínuo \\
\hline \#9 & RAD & 9 & 1.0 & 24.0 & $9.55 \pm 8.71$ & contínuo \\
\hline \#10 & TAX & 66 & 187.0 & 711.0 & $408.24 \pm 168.54$ & contínuo \\
\hline$\# 11$ & PTRAT1O & 46 & 12.6 & 22.0 & $I 8.45 \pm 2.16$ & contínuo \\
\hline \#12 & B & 357 & 0.32 & 396.9 & $356.67 \pm 91.29$ & contínuo \\
\hline \#13 & LSTAT & 455 & 1.73 & 37.97 & $12.65 \pm 7.14$ & contínuo \\
\hline \#14 & TARGET & 229 & 5.0 & 50.0 & $22.53 \pm 9.20$ & contínuo \\
\hline
\end{tabular}

Tabela 6.2: Descrição detalhada do conjunto de dados Boston Housing.

MPG Esse conjunto de dados foi retirado da biblioteca StatLib mantida pela Universidade Carnegie Mellon. Ele foi utilizado pela primeira vez na American Statistical Association Exposition em 15/08/1983, um fórum entre especialistas da indústria automobilística e estatísticos, na tentativa de resolver um problema das empresas automotivas que era a previsão da mpg (milles per gallon) de carros europeus, americanos e japoneses a fim de identificar fatores de maior consumo de combustível. O conjunto de dados é formado por 398 exemplos e 9 atributos ( 7 atributos contínuos e 2 atributos categóricos). Na Tabela 6.3 podem ser observadas algumas características detalhadas dos atributos desse conjunto de dados.

Ozone Devido à crescente concentração de pessoas em grandes centros urbanos, bem como a preocupação com a camada de ozônio, existe a preocupação em determinar a influência de fatores climáticos no aumento da degradação da camada de ozônio. Dentro desse contexto, o conjunto de dados Ozone é formado por dados meteorológicos coletados (temperatura, pressão, umidade, entre outras) em Los Angeles, CA. O conjunto de dados é formado por 366 exemplos e 10 atributos contínuos. Um fator preponderante nesse conjunto de 


\begin{tabular}{|c|c|c|c|c|c|c|c|}
\hline $\begin{array}{r}\text { Número do } \\
\text { atributo }\end{array}$ & $\begin{array}{l}\text { Nome do } \\
\text { atributo }\end{array}$ & $\begin{array}{c}\text { \% Vulores } \\
\text { ausentes }\end{array}$ & $\begin{array}{l}\text { \# Vulores } \\
\text { distintos }\end{array}$ & $\begin{array}{r}\text { Vulor } \\
\text { mínimo }\end{array}$ & $\begin{array}{r}\text { Vulor } \\
\text { máximo }\end{array}$ & MédiatDP & $\begin{array}{l}\text { Tipo do } \\
\text { atributo }\end{array}$ \\
\hline$\# 1$ & Cylinders & - & 5 & 3.0 & 8.0 & $5.47 \pm 1.69$ & contínuo \\
\hline$\# 2$ & Engine Displacement & - & 78 & 64.0 & 455.0 & $194.61 \pm 104.26$ & contínuo \\
\hline \#3 & Horsepower & - & 88 & 46.0 & 230.0 & $104.28 \pm 39.04$ & contínuo \\
\hline$\# 4$ & Weight & - & 288 & 1613.0 & 4997.0 & $2980.73 \pm 847.90$ & contínuo \\
\hline \#5 & Time 0 to 60 & - & 90 & 8.0 & 24.8 & $15.66 \pm 2.81$ & contínuo \\
\hline \#6 & Model Year & - & 13 & 70.0 & 82.0 & $75.91 \pm 3.71$ & contínuo \\
\hline \#7 & Origin & - & 3 & - & - & 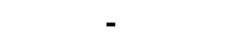 & categórico \\
\hline \#8 & Wagon? & - & $\dot{2}$ & 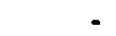 & - & - & categórico \\
\hline \#9 & Miles per Gullon & - & 111 & 9.0 & 46.6 & $23.49 \pm 7.92$ & contínuo \\
\hline
\end{tabular}

Tabela 6.3: Descrição detalhada do conjunto de dados MPG.

dados é a existência de valores ausentes. $\mathrm{Na}$ Tabela 6.4 podem ser observadas algumas características desse conjunto de dados.

\begin{tabular}{r|l|c|lrr|r|r}
\hline \hline $\begin{array}{r}\text { Número do } \\
\text { atributo }\end{array}$ & $\begin{array}{l}\text { Nome do } \\
\text { atributo }\end{array}$ & $\begin{array}{c}\text { \% Vulores } \\
\text { uusentes }\end{array}$ & $\begin{array}{l}\text { \# Valores } \\
\text { distintos }\end{array}$ & $\begin{array}{r}\text { Vulor } \\
\text { minimo }\end{array}$ & $\begin{array}{r}\text { Vulor } \\
\text { múximo }\end{array}$ & Média \pm DP & $\begin{array}{r}\text { Tipo do } \\
\text { utributo }\end{array}$ \\
\hline$\# 1$ & VDB500HT & 3 & 53 & 5320 & 5950 & $5752.97 \pm 104.99$ & contínuo \\
$\# 2$ & WDSP & - & 11 & 0 & 11 & $4.87 \pm 2.12$ & contínuo \\
contínuo \\
$\# 3$ & HMDTY & 4 & 67 & 19 & 93 & $58.48 \pm 19.76$ & contínuo \\
$\# 4$ & SDAFBTMP & 1 & 64 & 25 & 93 & $61.91 \pm 14.28$ & contínuo \\
$\# 5$ & EMAFBTMP & 38 & 147 & 27.68 & 82.58 & $56.85 \pm 11.66$ & continuo \\
$\# 6$ & INVHT & 4 & 208 & 111 & 5000 & $2590.94 \pm 1796.83$ & continuo \\
$\# 7$ & DAGPG & $<1$ & 132 & -69 & 107 & $17.80 \pm 36.11$ & contínuo \\
$\# 8$ & INVTMP & 4 & 199 & 27.5 & 91.76 & $60.93 \pm 13.87$ & contínuo \\
$\# 9$ & VZBLTY & - & 25 & 0 & 500 & $123.30 \pm 80.28$ & contínuo \\
contínuo
\end{tabular}

Tabela 6.4: Descriçāo detalhada do conjunto de dados Ozone.

Permeability A permeabilidade de poços de petróleo é um atributo-meta a empresas que lucram com petróleo, e é de interesse que a perda de petróleo seja mínima. Nesse contexto, esse conjunto de dados concentra-se no problema de predizer a permeabilidade de poços de petróleo em Big Escambia Creek - Alabama/EUA, segundo as medidas de profundidade e porosidade medidos. Esse conjunto de dados é formado por 982 registros e 3 atributos contínuos [Rogers et al., 1995] e foi utilizado em outros trabalhos no LABIC [Félix et al., 2000, Félix et al., 1998b]. Na Tabela 6.5 podem ser observadas algumas características do conjunto de dados Permeability dividido em 3 cenários nos experimentos realizados em [Rogers et al., 1995].

PMGRN-455 O PMGRN-455 é um subconjunto da Base de Dados do PMGRN (Programa de Melhoramento Genético da Raça Nelore) do Departamento de Genética da Faculdade de Medicina de Ribeirão Preto [Lôbo et al., 1999]. Essa base é constituída de mais de 177000 animais cadastrados com 81 atributos referentes a características de cada animal. O conjunto de dados consiste de 6599 animais (3216 machos e 3383 fêmeas) nascidos entre os anos de 1987 e 1996, com 8 atributos contínuos. A característica a ser predita nesse subconjunto é o peso aos 455 dias que interessa ao Especialista do Domínio, devido a 


\begin{tabular}{|c|c|c|c|c|c|c|}
\hline $\begin{array}{r}\text { Número do } \\
\text { atributo }\end{array}$ & $\begin{array}{l}\text { Nome do } \\
\text { atributo }\end{array}$ & $\begin{array}{l}\text { \# Vulores } \\
\text { distintos }\end{array}$ & $\begin{array}{r}\text { Vulor } \\
\text { mínimo }\end{array}$ & $\begin{array}{r}\text { Valor } \\
\text { maximo }\end{array}$ & Média $\pm D P$ & $\begin{array}{l}\text { Tipo do } \\
\text { atributo }\end{array}$ \\
\hline \multicolumn{7}{|l|}{ Scenariol } \\
\hline $\begin{array}{l}\# 1 \\
\# 2 \\
\# 3\end{array}$ & $\begin{array}{l}\text { Depth } \\
\text { Porosity } \\
\text { Permeability }\end{array}$ & $\begin{array}{l}345 \\
180 \\
112\end{array}$ & $\begin{array}{r}15254 \\
0.6 \\
0.0090\end{array}$ & $\begin{array}{r}15658 \\
27.2 \\
44.0\end{array}$ & $\begin{array}{c}15470.51 \pm 101.34 \\
8.14 \pm 6.33 \\
2.42 \pm 6.01\end{array}$ & $\begin{array}{l}\text { contínuo } \\
\text { continuo } \\
\text { contínuo }\end{array}$ \\
\hline \multicolumn{7}{|l|}{ Scenurio2 } \\
\hline $\begin{array}{l}\# 1 \\
\# 2 \\
\# 3\end{array}$ & $\begin{array}{l}\text { Depth } \\
\text { Porosity } \\
\text { Permeubility }\end{array}$ & $\begin{array}{l}346 \\
196 \\
129\end{array}$ & $\begin{array}{r}15254 \\
0.6 \\
0.0090\end{array}$ & $\begin{array}{r}15658 \\
27.2 \\
49.0\end{array}$ & $\begin{array}{c}15477.59 \pm 93.81 \\
9.02 \pm 6.37 \\
3.25 \pm 7.44\end{array}$ & $\begin{array}{l}\text { contínuo } \\
\text { continuo } \\
\text { contínuo }\end{array}$ \\
\hline \multicolumn{7}{|l|}{ Scenario3 } \\
\hline $\begin{array}{l}\# 1 \\
\# 2 \\
\# 3\end{array}$ & $\begin{array}{l}\text { Depth } \\
\text { Porosity } \\
\text { Permesbility }\end{array}$ & $\begin{array}{l}447 \\
201 \\
132 \\
\end{array}$ & $\begin{array}{r}15124 \\
0.6 \\
0.0090\end{array}$ & $\begin{array}{r}15658 \\
27.2 \\
44.0 \\
\end{array}$ & $\begin{array}{c}15440.64 \pm 132.40 \\
8.28 \pm 6.33 \\
2.77 \pm 6.93\end{array}$ & $\begin{array}{l}\text { contínuo } \\
\text { continuo } \\
\text { contínuo }\end{array}$ \\
\hline
\end{tabular}

Tabela 6.5: Descrição detalhada do conjunto de dados Permeability.

algumas suspeitas de ser um bom preditor do peso aos 550 dias. Na Tabela 6.6 podem ser observadas características detalhadas do PMGRN-455.

\begin{tabular}{|c|c|c|c|c|c|c|}
\hline $\begin{array}{r}\text { Número do } \\
\text { utributo }\end{array}$ & $\begin{array}{l}\text { Nome do } \\
\text { utrihuto }\end{array}$ & $\begin{array}{l}\text { \# Vulores } \\
\text { distintos }\end{array}$ & $\begin{array}{r}\text { Vulor } \\
\text { mínimo }\end{array}$ & $\begin{array}{l}\text { Valor } \\
\text { máximo }\end{array}$ & Média $\pm D P$ & $\begin{array}{l}\text { Tipo do } \\
\text { utributo }\end{array}$ \\
\hline \#1 & $\mathrm{m} 120$ & 1 & $\overline{1}$ & $\bar{I}$ & $1 \pm 0$ & contínuo \\
\hline \#2 & pl20 & 122 & 55 & 202 & $117.90 \pm 17.77$ & contínuo \\
\hline$\# 3$ & $\mathrm{~m} 240$ & 1 & 1 & 1 & $1 \pm 0$ & contínuo \\
\hline \#4 & p240 & 176 & 80 & 304 & $185.17 \pm 27.50$ & continuo \\
\hline \#5 & m365 & 1 & 1 & 1 & $1 \pm 0$ & contínuo \\
\hline \#6 & p365 & 225 & 93 & 399 & $212.16 \pm 33.71$ & contínuo \\
\hline \#7 & $m 455$ & 2 & 1 & 2 & $1.03 \pm 0.06$ & contínuo \\
\hline \#8 & p455 & 267 & 106 & 491 & $248.19 \pm 42.75$ & contínuo \\
\hline
\end{tabular}

Tabela 6.6: Descrição detalhada do conjunto de dados PMGRN-455.

\subsubsection{Síntese Geral das Características dos Conjuntos de Dados}

$\mathrm{Na}$ Tabela 6.7 pode ser observada uma síntese dos conjuntos de dados apresentados em ordem crescente de acordo com o número de atributos, tais como: número de instâncias (exemplos), instâncias duplicadas ou conflitantes (valor percentual), atributos (número e tipos) e se o conjunto de dados possui valores ausentes.

\begin{tabular}{c|cc|c}
\hline \hline Dutaset & \# Instâncius & $\begin{array}{c}\text { \# Atributos } \\
\text { (cont., cat.) }\end{array}$ & $\begin{array}{c}\text { Vulores } \\
\text { Ausentes }\end{array}$ \\
\hline Permeability & 982 & $3(3,0)$ & $\mathrm{N}$ \\
CPU & 209 & $7(7,0)$ & $\mathrm{N}$ \\
PMGRN-455 & 6599 & $8(8,0)$ & $\mathrm{N}$ \\
MPG & 398 & $9(7,2)$ & $\mathrm{N}$ \\
Ozone & 366 & $10(10,0)$ & $\mathrm{S}$ \\
Housing & 506 & $14(14,0)$ & $\mathrm{N}$ \\
\hline \hline
\end{tabular}

Tabela 6.7: Síntese dos conjuntos de dados.

Na Figura 6.1 podem ser observadas as dimensionalidades dos conjuntos de dados (eixo X), o 
número de instâncias (eixo Y à esquerda no gráfico) e o número de atributos de cada conjunto de dados (eixo Y à direita do gráfico). Sendo que, o número de instâncias está reduzido a $\log _{10}$ (\# Instâncias).

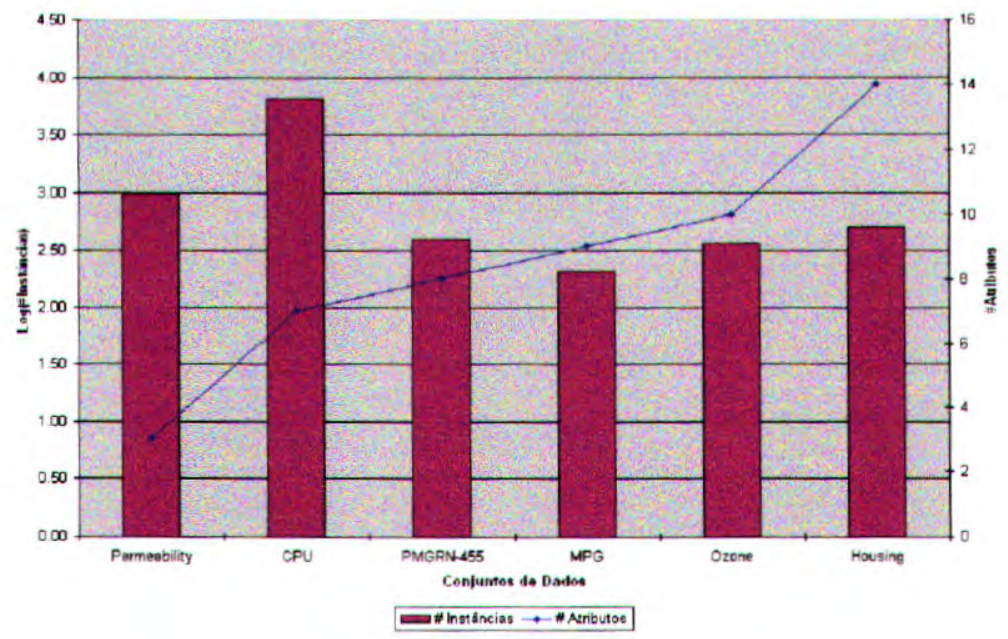

Figura 6.1: Dimensionalidade dos conjuntos de dados — Instâncias $\times$ Atributos.

\subsection{Descrição dos Experimentos Realizados}

Para cada conjunto de dados, os experimentos foram realizados de acordo com o esquema apresentado na Figura 6.2.

O processo inicia-se com a Preparação dos Dados de modo que esses dados possam ser fornecidos a um algoritmo de aprendizado. Nesta fase apenas alguns conjuntos de dados tiveram seus dados preparados. Em seguida, os dados preparados são apresentados aos algoritmos Cubist e M5 para realizar a extração de padrões. O resultado dessa etapa é o conhecimento extraído na forma de regras e árvores respectivamente. O conhecimento extraído então é avaliado no

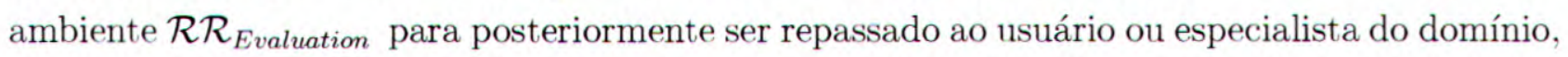
com a indicação, entre outras, das regras mais relevantes.

As atividades realizadas em uma dessas etapas são descritas a seguir. 


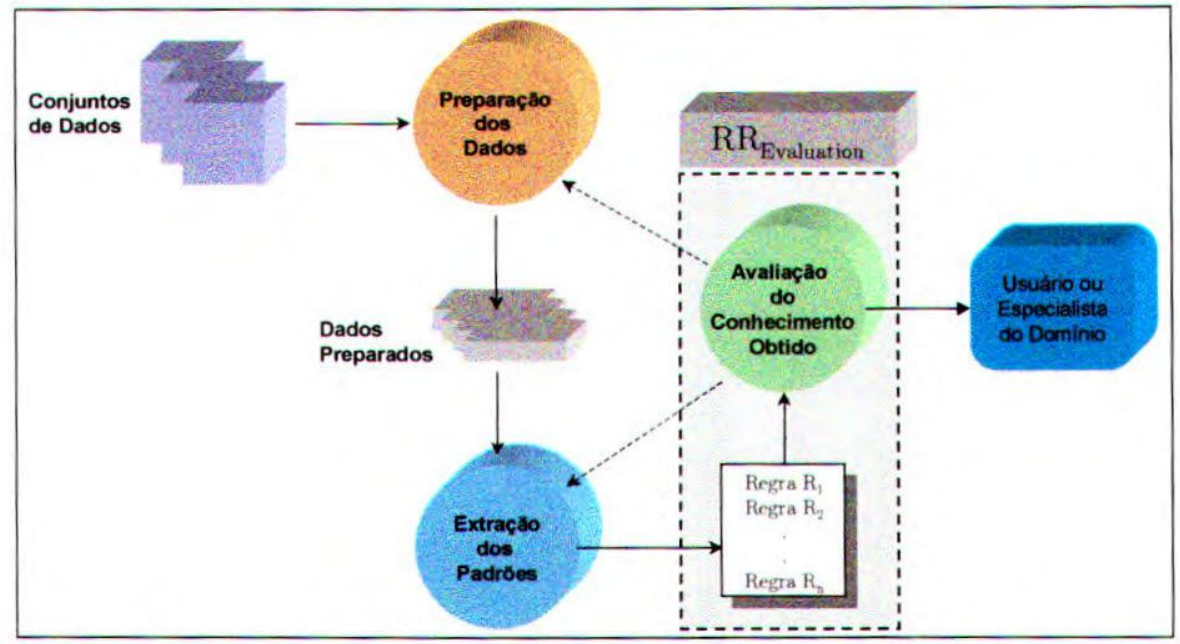

Figura 6.2: Diagrama de estados utilizado na realização dos experimentos.

\subsubsection{Preparação dos Dados}

Em alguns dos conjuntos de dados (CPU, Ozone, Permeability e PMGRN-455) foi necessária uma preparação de seus dados (seleção e transformação dos dados).

No conjunto de dados CPU foi retirado o atributo vendor, que possui como valores o nome do vendedor. Por se tratar de uma característica muito específica, o atributo foi desconsiderado nos experimentos.

No conjunto de dados Ozone foram observados que quase todos os atributos possuem valores ausentes (missing values), exceto HMDTY e VZBLTY. O atributo EMAFBTMP, em especial, no conjunto de dados possui $38 \%$ com valor ausente. Nesses experimentos todas as instâncias que possuíam valores ausentes foram retiradas. Essa exclusão deve-se, principalmente, a uma limitação do ambiente $\mathcal{R}_{\mathcal{R}_{\text {Evaluation }}}$ que, ainda, não possui mecanismos de tratamento para valores ausentes.

No conjunto de dados Permeability foram selecionados dentre os 982 exemplos, 3 cenários com diferentes números de exemplos. Essa divisão do conjunto de dados em cenários foi proposta no trabalho de [Rogers et al., 1995] e também utilizada nos experimentos dos trabalhos [Félix et al., 2000, Félix et al., 1999, Félix et al., 1998b].

No conjunto de dados PMGRN-455 foram observados 8 atributos contínuos e 1 atributo categórico (multivalorado) que consiste no número da fazenda. Por ser uma característica específica, referentes aos números das fazendas, o atributo foi desconsiderado nos experimentos. 
Todos os conjuntos de dados foram colocados nos formatos do Cubist e do $\mathcal{M} 5$.

\subsubsection{Extração de Padrões}

Nessa seção são feitas algumas descrições das regras extraídas pelos algoritmos Cubist e $\mathcal{M} 5$ nos conjuntos de dados descritos. Como o $\mathcal{M} 5$ gera árvores de regressão as regras foram traduzidas das árvores de regressão utilizando o $\mathcal{M} 5_{2}$ Rules.

\section{CPU}

Nas Figuras 6.3 e 6.4 podem ser observadas as regras geradas pelo Cubist e pelo $\mathcal{M} 5$. Por exemplo, para o Cubist a primeira regra na Figura 6.3, Rule 1, especifica que se CHMIN $<=7$ então o atributo-meta PERFORMANCE recebe um valor resultante do modelo linear $9.2+0.00326$ MMAX + $0.83 \mathrm{CACH}+0.0015$ MMIN + 0.1 CHMAX + $0.004 \mathrm{MYCT}$, cobrindo 165 exemplos. A média do valor do atributo-meta é igual a 50.5, o valor mínimo e máximo são iguais a 6 e 259 respectivamente e o erro estimado é de 18.3 .

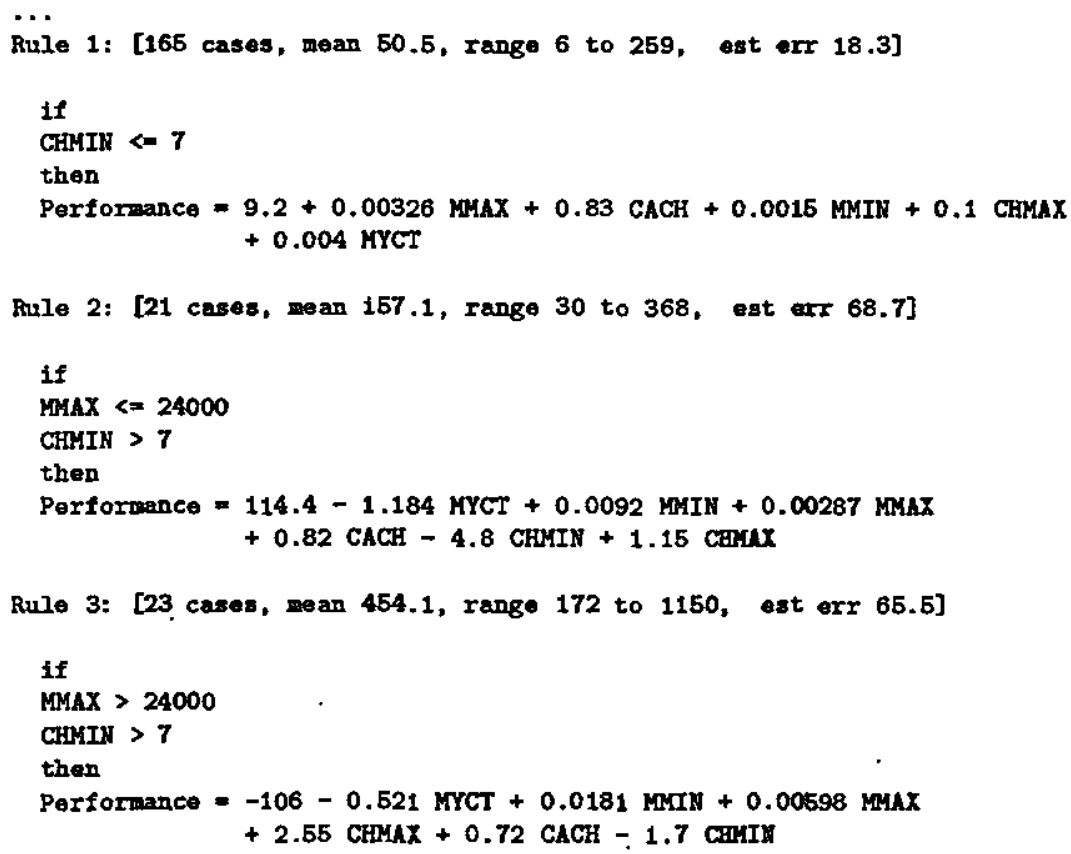

Figura 6.3: Regras obtidas pelo Cubist para o conjunto de dados CPU. 
na Figura 6.4 especifica que se CHMIN < 7.5 então o modelo linear LM1 pode ser utilizado para predizer o atributo-meta PERFORMANCE. A segunda regra especifica que se CHMIN > 7.5 e MMAX $<28000$ o modelo linear LM2 pode ser utilizado e assim por diante.

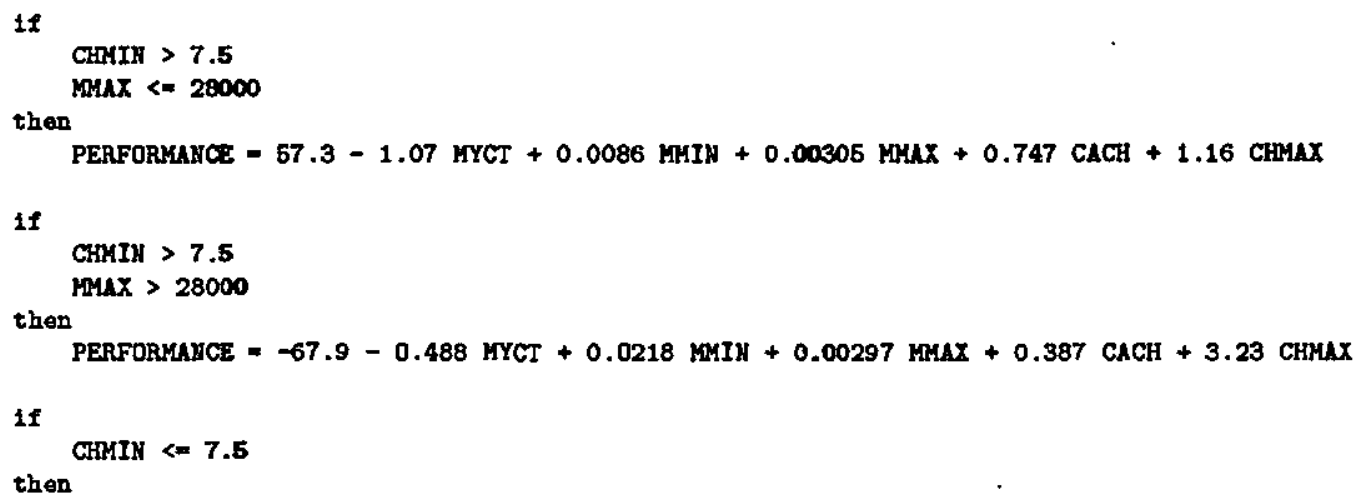

Figura 6.4: Regras obtidas pelo $\mathcal{M} 5_{2}$ Rules para o conjunto de dados CPU.

$\mathrm{Na}$ Tabela 6.8 podem ser observadas as mesmas regras em formato de atributo-valor. Esse formato visa facilita a análise dos atributos presentes no modelo linear utilizado para as predições de cada regra.

\begin{tabular}{|c|c|c|c|c|c|c|c|c|c|c|}
\hline \multirow{2}{*}{$\begin{array}{c}\# \\
\text { Regra }\end{array}$} & \multirow{2}{*}{$\begin{array}{l}\# \\
\text { Exs: }\end{array}$} & \multicolumn{2}{|c|}{$\overline{\text { Attributos }}$} & \multirow[b]{2}{*}{ Constante } & \multirow[b]{2}{*}{ MYCT } & \multicolumn{2}{|c|}{ Preditores } & \multirow[b]{2}{*}{$\mathrm{CACH}$} & \multirow[b]{2}{*}{ CHMIN } & \multirow[b]{2}{*}{ CHMAX } \\
\hline & & MMAX & CHMIN & & & MMIN & MMAX & & & \\
\hline \multicolumn{11}{|l|}{ Cubist } \\
\hline$\overline{\mathbf{R}_{1}}$ & 165 & & $<=7$ & 9.2 & 0.004 & 0.0015 & 0.00326 & 0.83 & & \\
\hline $\mathbf{R}_{\mathbf{1}}$ & 21 & $<=24000$ & $>7$ & 114.4 & -1.184 & 0.0092 & 0.00287 & 0.82 & -4.8 & 1.15 \\
\hline $\mathbf{R}_{2}$ & 23 & $>24000$ & $>7$ & -106 & .0521 & 0.0181 & 0.00598 & 2.55 & -1.7 & 0.72 \\
\hline \multicolumn{11}{|l|}{$\mathcal{M 5}$} \\
\hline $\overrightarrow{\mathbf{R}_{1}}$ & 165 & & $<=7.5$ & 7.34 & 0.00409 & 0.00127 & 0.00305 & 0.82 & & 0.424 \\
\hline $\mathbf{R}_{2}$ & 21 & $<=28000$ & $>7.5$ & 57.3 & -1.07 & 0.0086 & 0.00305 & 0.75 & & 1.16 \\
\hline $\mathrm{R}_{3}$ & 23 & $<28000$ & $>7.5$ & -67.3 & -0.488 & 0.0218 & 0.00297 & 0.39 & & 3.23 \\
\hline
\end{tabular}

Tabela 6.8: Atributos e Preditores das regras obtidas pelo Cubist e pelo $\mathcal{M} 5$.

Nos demais conjuntos de dados as regras de regressão estão apresentadas somente no formato atributo-valor como na Tabela 6.8 .

\section{Boston Housing}

Utilizando-se o algoritmo Cubist no conjunto de dados Boston Housing foram obtidas dez regras de regressão, enquanto no $\mathcal{M} 5$ foram obtidas dezoito regras. Na Tabela $6.9^{1}$ e na Tabela 6.10

\footnotetext{
${ }^{1}$ A Tabela 6.10 consiste das equações pertencentes as regras da Tabela 6.9.
} 
podem ser observadas as descrições dos atributos presentes nas condiçōes das regras do Cubist e as funções matemáticas pertinentes a cada uma delas, respectivamente.

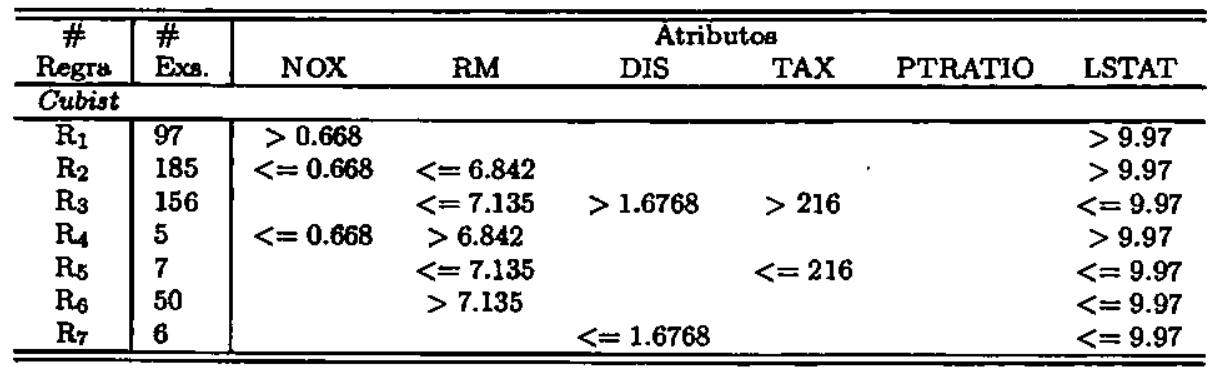

Tabela 6.9: Condições das regras obtidas pelo Cubist para o conjunto de dados Boston Housing.

\begin{tabular}{|c|c|c|c|c|c|c|c|c|c|c|c|c|}
\hline \multirow{2}{*}{$\begin{array}{c}\# \\
\text { Regra }\end{array}$} & \multicolumn{12}{|c|}{ Preditores } \\
\hline & Constante & NOX & $\mathbf{R M}$ & DIS & TAX & PTRATIO & LSTAT & AGE & CRIM & $\mathrm{RAD}$ & B & INDUS \\
\hline \multicolumn{13}{|l|}{ Cubist } \\
\hline$\overline{\mathbf{R}_{1}}$ & -2.06 & 21.8 & 0.23 & 2.99 & -0.0006 & -0.14 & -0.326 & -0.003 & -0.018 & 0.011 & 0.0078 & \\
\hline $\mathbf{R}_{2}$ & 36.24 & -1.6 & 0.44 & -0.58 & -0.0006 & -0.67 & -0.265 & -0.035 & -0.011 & 0.011 & 0.0082 & -0.006 \\
\hline $\mathbf{R}_{3}$ & -8.41 & -1.1 & 7.66 & -0.52 & -0.0008 & -0.41 & -0.577 & -0.038 & -0.006 & 0.106 & 0.0006 & \\
\hline $\mathrm{R}_{4}$ & 21.62 & 7.5 & 2.22 & -0.65 & -0.0006 & -0.53 & -0.109 & -0.034 & -0.011 & 0.011 & 0.0068 & -0.06 \\
\hline$R_{5}$ & 9.5 & -1.1 & 5.56 & -0.51 & -0.0008 & -0.35 & -0.528 & -0.015 & -0.006 & 0.106 & 0.0006 & \\
\hline $\mathbf{R}_{6}$ & 16.54 & -1.1 & 7.86 & -0.22 & -0.0286 & -1.22 & -1.656 & & -0.006 & 0.127 & 0.0006 & \\
\hline $\mathbf{R}_{7}$ & 33.67 & -1.1 & 4.58 & -12.27 & -0.0008 & -0.13 & -1.092 & & -0.006 & 0.521 & 0.0006 & \\
\hline
\end{tabular}

Tabela 6.10: Funções matemáticas para regra obtida pelo Cubist no conjunto de dados Boston Housing.

As regras obtidas pelo $\mathcal{M} 5$ e as funçōes matemáticas obtidas para cada regra podem ser observadas nas Tabelas 6.11 e 6.12 , respectivamente.

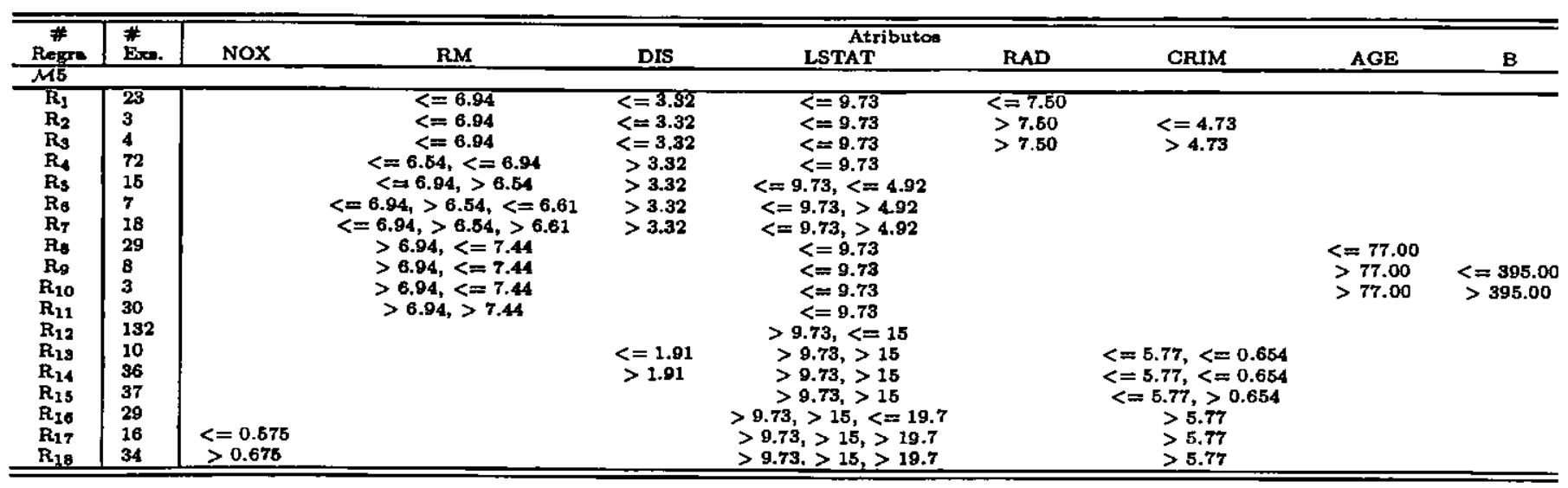

Tabela 6.11: Condições das regras obtidas pelo $\mathcal{M} 5$ para o conjunto de dados Boston Housing.

\section{MPG}

Para o conjunto de dados MPG, as regras encontradas pelos algoritmos Cubist e $\mathcal{M} 5$ foram combinadas em uma única tabela (Tabela 6.13). As equaçōes matemáticas das regras encontradas por cada algoritmo foram separadas em duas tabelas. Na Tabela 6.14 podem ser observadas 


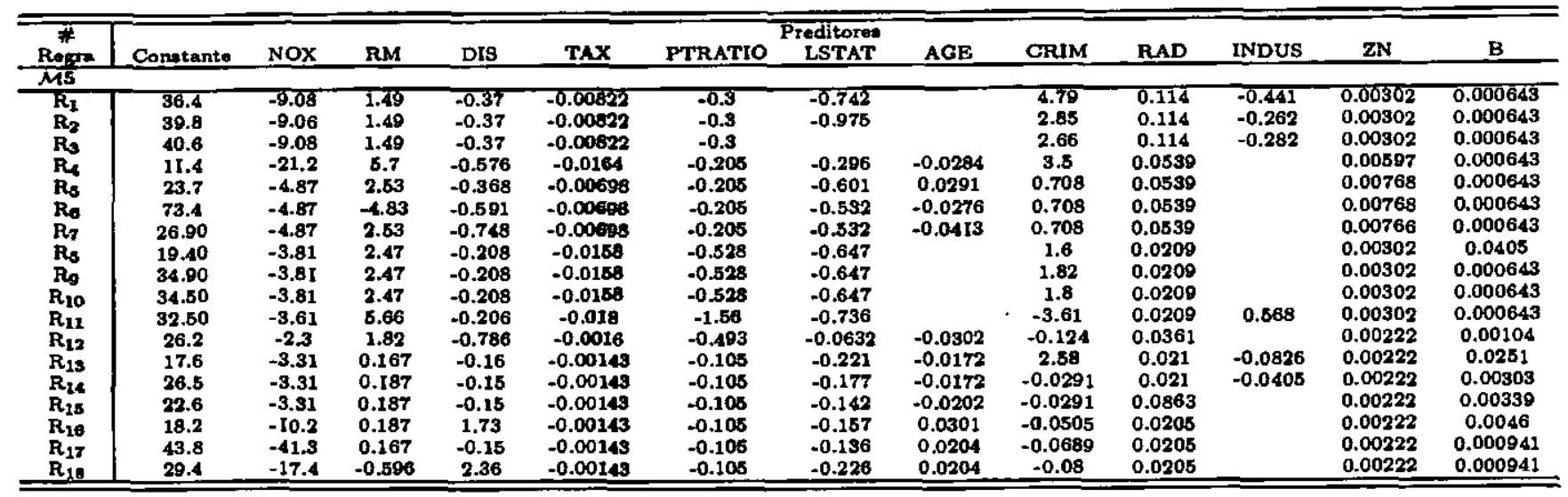

Tabela 6.12: Funçōes matemáticas para regra obtida pelo $\mathcal{M} 5$ no conjunto de dados Boston Housing.

as equações matemáticas geradas pelo Cubist, enquanto na Tabela 6.15 podem ser observadas as equações matemáticas geradas pelo $\mathcal{M} 5$.

\begin{tabular}{|c|c|c|c|c|c|}
\hline \multirow{2}{*}{$\begin{array}{c}\# \\
\text { Regru }\end{array}$} & \multirow{2}{*}{$\begin{array}{l}\text { \# } \\
\text { Exs. }\end{array}$} & \multicolumn{4}{|c|}{ Atributos } \\
\hline & & CYLINDERS & ENGINE & HORSEPOWER & MODELYEAR \\
\hline \multicolumn{6}{|c|}{ Cubist } \\
\hline $\begin{array}{l}\mathrm{R}_{1} \\
\mathrm{R}_{2}\end{array}$ & $\begin{array}{l}152 \\
67\end{array}$ & $\begin{array}{c}<=5 \\
>5\end{array}$ & & $>70$ & $<=78$ \\
\hline $\mathrm{R}_{3}$ & 25 & & & $<=70$ & $<=77$ \\
\hline$R_{4}$ & 41 & $<=5$ & $\cdot$ & $>70$ & $>78$ \\
\hline $\mathrm{R}_{5}$ & 35 & & & $<=70$ & $>77$ \\
\hline \multicolumn{6}{|l|}{$\mathcal{M} 5$} \\
\hline $\mathbf{\mathbf { R } _ { 1 }}$ & 180 & & $<=191$ & & \\
\hline $\mathbf{R}_{2}$ & 37 & & $>191,<=241$ & $<=127$ & \\
\hline $\mathbf{R}_{3}$ & 19 & & $>191,>241,<=259$ & $<=127$ & \\
\hline $\mathbf{R}_{4}$ & 7 & & $>191,>241,>259$ & $<=127$ & \\
\hline $\mathbf{R}_{\mathbf{5}}$ & 77 & & $>191$ & $>127$ & \\
\hline
\end{tabular}

Tabela 6.13: Condições das regras obtidas utilizando os algoritmos Cubist e $\mathcal{M} 5$ no conjunto de dados MPG.

\begin{tabular}{|c|c|c|c|c|c|c|}
\hline \multirow{2}{*}{$\begin{array}{c}\# \\
\text { Regra }\end{array}$} & \multicolumn{6}{|c|}{ Preditores } \\
\hline & Constante & WEIGHT & MODELYEAR & CYLINDERS & ENGINE & HORSEPOWER \\
\hline \multicolumn{7}{|l|}{ Cubist } \\
\hline $\mathbf{R}_{1}$ & 20.54 & -0.0068 & 0.27 & 0.48 & -0.0059 & -0.007 \\
\hline $\mathbf{R}_{2}$ & -2.19 & -0.00419 & 0.46 & & & \\
\hline $\mathbf{R}_{3}$ & -28.28 & .0 .00161 & 0.92 & 2.12 & -0.1445 & -0.044 \\
\hline $\mathbf{R}_{4}$ & 8.66 & -0.00834 & 0.32 & 5.21 & -0.0278 & -0.007 \\
\hline $\mathbf{R}_{5}$ & 11.15 & -0.00161 & 0.47 & 1.85 & -0.0419 & -0.199 \\
\hline
\end{tabular}

Tabela 6.14: Equações matemáticas para cada regra obtida pelo algoritmo Cubist no conjunto de dados MPG.

\section{Ozone}

Nos experimentos com o conjunto de dados Ozone, os algoritmos Cubist e $\mathcal{M} 5$ utilizaram dados sem $\propto$ valores ausentes contidos no conjunto original. Na Tabela 6.16 podem ser observadas as 


\begin{tabular}{|c|c|c|c|c|c|c|c|}
\hline \multirow{2}{*}{$\begin{array}{c}\# \\
\text { Regra }\end{array}$} & \multicolumn{7}{|c|}{ Preditores } \\
\hline & Constante & WEIGHT & MODELYEAR & ENGINE & HORSEPOWER & TIME & ORIGIN \\
\hline \multicolumn{8}{|l|}{$\mathcal{M 5}$} \\
\hline $\mathbf{R}_{1}$ & -18.3 & -0.00728 & 0.894 & & -0.0703 & & 1.66 origin in \{Europe,Japun\} \\
\hline $\mathrm{R}_{2}$ & 1.64 & -0.00418 & 0.419 & & & & 0.205 origin in $\{$ Europe,Jupan \\
\hline $\mathbf{R}_{3}$ & -6.33 & -0.0027 & 0.614 & & -0.0528 & -0.407 & 0.205 origin in $\{$ Europe,Jupen \\
\hline $\mathbf{R}_{4}$ & -4.87 & -0.0027 & 0.774 & & -0.162 & -0.468 & 0.205 origin in $\{$ Europe,Japun \\
\hline $\mathrm{R}_{5}$ & -1.97 & -0.00118 & 0.475 & -0.019 & & -0.559 & 0.205 origin in \{Europe,Jupan \\
\hline
\end{tabular}

Tabela 6.15: Equações matemáticas para cada regra obtida pelo algoritmo $\mathcal{M} 5$ no conjunto de dados MPG.

regras geradas, juntamente com as condições das regras e na Tabela 6.17 podem ser observadas as equaçōes matemáticas das regras obtidas.

\begin{tabular}{|c|c|c|c|c|c|c|}
\hline \multirow{2}{*}{$\begin{array}{c}\# \\
\text { Regra }\end{array}$} & \multirow{2}{*}{$\begin{array}{l}\# \\
\text { Exs. }\end{array}$} & \multicolumn{5}{|c|}{ Atributos } \\
\hline & & HMDTY & EMAFBTMP & INVHT & DAGPG & INVTMP \\
\hline \multicolumn{7}{|l|}{ Cubist } \\
\hline $\begin{array}{l}R_{1} \\
R_{2}\end{array}$ & $\begin{array}{l}31 \\
17\end{array}$ & $<=71$ & $\begin{array}{l}<=62.24 \\
<=62.24\end{array}$ & $>1571$ & $\begin{array}{l}>-21 \\
<=-21\end{array}$ & \\
\hline $\begin{array}{l}\mathrm{R}_{3} \\
\mathrm{R}_{4}\end{array}$ & $\begin{array}{l}52 \\
8\end{array}$ & $\begin{array}{l}>71 \\
<=51\end{array}$ & $\begin{array}{l}<=62.24 \\
>62.24\end{array}$ & $>1571$ & & \\
\hline $\mathrm{R}_{5}$ & 37 & & $<=62.24$ & $<=1571$ & $>-21$ & \\
\hline $\mathbf{R}_{5}$ & 11 & & $>62.24$ & & $>54$ & \\
\hline $\mathbf{R}_{\mathbf{7}}$ & 47 & $>51$ & $>62.24$ & & $<=54$ & \\
\hline \multicolumn{7}{|l|}{$\mathcal{M} 5$} \\
\hline$\overline{\mathrm{R}_{1}}$ & 58 & & $<=62.9$ & & & $<=52.6$ \\
\hline $\mathrm{R}_{2}$ & 2I & & $<=62.9$ & & $<=-23$ & $>52.6$ \\
\hline $\mathbf{R}_{3}$ & 61 & & $<=62.9$ & & $>-23$ & $>52.6$ \\
\hline $\mathbf{R}_{4}$ & 63 & & $>62.9$ & - & & \\
\hline
\end{tabular}

Tabela 6.16: Condições das regras obtidas utilizando os algoritmos Cubist e $\mathcal{M} 5$ para o conjunto de dados Ozone.

\begin{tabular}{|c|c|c|c|c|c|c|c|}
\hline \multirow{2}{*}{$\begin{array}{c}\# \\
\text { Regra }\end{array}$} & \multicolumn{7}{|c|}{ Preditores } \\
\hline & Constante & EMAFBTMP & INVTMP & INVHT & SDAFBTMP & HMDTY & DAGPG \\
\hline \multicolumn{8}{|l|}{ Cubist } \\
\hline$\overline{\mathbf{R}_{\mathbf{1}}}$ & -2.08 & 0.271 & -0.095 & -0.0005 & 0.048 & 0.01 & 0.002 \\
\hline $\mathbf{R}_{2}$ & 2.3 & 0.13 & -0.115 & -0.00059 & 0.066 & 0.01 & 0.006 \\
\hline $\mathbf{R}_{3}$ & -6.41 & 0.307 & -0.095 & -0.0005 & 0.048 & 0.01 & 0.002 \\
\hline $\mathrm{R}_{4}$ & -38.5 & 0.662 & -0.042 & -0.00023 & 0.03 & 0.159 & -0.02 \\
\hline $\mathbf{R}_{5}$ & -2.18 & 0.552 & -0.315 & -0.00063 & 0.056 & 0.01 & 0.002 \\
\hline $\mathrm{R}_{6}$ & -17.35 & 0.46 & -0.042 & -0.00023 & 0.03 & 0.06 & -0.025 \\
\hline $\mathbf{R}_{7}$ & -29.94 & 0.749 & -0.042 & -0.00023 & -0.073 & 0.128 & -0.013 \\
\hline \multicolumn{8}{|l|}{ M5 } \\
\hline$\overline{\mathrm{R}_{1}}$ & -0.994 & 0.108 & -0.0814 & -0.000429 & 0.126 & 0.00989 & 0.0048 \\
\hline $\mathbf{R}_{2}$ & -0.692 & 0.19 & -0.0665 & -0.000625 & $0.0351 \mathrm{P}$ & 0.00989 & 0.0238 \\
\hline $\mathbf{R}_{3}$ & -6.53 & 0.343 & -0.0665 & -0.000481 & 0.0351 & 0.00989 & 0.0132 \\
\hline $\mathbf{R}_{4}$ & -36.1 & 0.638 & -0.0418 & -0.000225 & 0.0295 & 0.187 & \\
\hline
\end{tabular}

Tabela 6.17: Equações matemáticas das regras obtidas pelos algoritmos Cubist e $\mathcal{M} 5$ para o conjunto de dados Ozone.

\section{Permeability}

O conjunto de dados Permeability, como ressaltado anteriormente, foi dividido em 3 cenários como proposto em [Félix et al., 1998b]. Cada um desses cenários possuem informações de 
diferentes poços de petróleo. O primeiro cenário possui 561 exemplos de treinamento, o segundo cenário possui 694 exemplo e o terceiro cenário possui 823 exemplos. Os algoritmos Cubist e $\mathcal{M} 5$ foram utilizados para cada um dos cenários propostos. Nas Tabelas $6.18,6.19$ e 6.20 podem ser observadas as regras e as funções matemáticas obtidas pelos algoritmos Cubist e $\mathcal{M} 5$ para cada um dos cenários respectivamente.

\begin{tabular}{|c|c|c|c|c|c|c|}
\hline \multirow{2}{*}{$\begin{array}{c}\# \\
\text { Regra }\end{array}$} & \multirow{2}{*}{$\begin{array}{l}\text { \# } \\
\text { Exxs. }\end{array}$} & \multicolumn{2}{|c|}{ Atributos } & \multicolumn{3}{|c|}{ Preditores } \\
\hline & & DEPTH & POROSITY & Constante & POROSITY & DEPTH \\
\hline \multicolumn{7}{|c|}{ Cubist } \\
\hline$\overline{\mathrm{R}_{1}}$ & 196 & $<=15594$ & $>2.9,<=12.1$ & -1.58 & 0.017 & 0.0001 \\
\hline $\mathbf{R}_{2}$ & 202 & & $<=2.9$ & -1.85 & 0.017 & 0.0001 \\
\hline $\mathrm{R}_{3}$ & 34 & $<=15505$ & $>12.1,<=15.2$ & -60.5 & 0.478 & 0.0036 \\
\hline $\mathbf{R A}_{\mathbf{4}}$ & 24 & $>15546,<=15591$ & $>12.1,<=16.8$ & 257.47 & 1.429 & -0.0177 \\
\hline $\mathbf{R}_{\boldsymbol{\sigma}}$ & 18 & $>15439,<=15505$ & $>15.2$ & -94.51 & 1.078 & 0.0051 \\
\hline $\mathbf{R}_{8}$ & 20 & $>15260,<=15424$ & $>15.2$ & -358.61 & 1.151 & 0.0224 \\
\hline $\mathbf{R}_{7}$ & 15 & $>15505,<=15546$ & $>12.1,<=16.2$ & -3129.99 & 2.351 & 0.1998 \\
\hline $\mathbf{R}_{8}$ & 5 & $>15594$ & $>2.9,<=12.1$ & -4457.41 & 0.017 & 0.2864 \\
\hline$R_{\theta}$ & 5 & $<=15260$ & $>15.2$ & -300.98 & 1.277 & 0.0187 \\
\hline $\mathrm{R}_{10}$ & 7 & $>15546$ & $>16.8$ & 3621.35 & 1.659 & -0.2339 \\
\hline $\mathrm{R}_{11}$ & 9 & $>15505,<=15527$ & $>16.2$ & -1704.84 & 2.582 & 0.1077 \\
\hline $\mathbf{R}_{12}$ & 12 & $>15424,<=15439$ & $>15.2$ & -199.11 & 1.362 & 0.0124 \\
\hline $\mathbf{R}_{13}$ & 5 & $>15591$ & $>12.1$ & 2569.99 & 1.166 & -0.1644 \\
\hline $\mathbf{R}_{14}$ & 9 & $>15527,<=15546$ & $>16.2$ & -2638.12 & 2.982 & 0.1681 \\
\hline \multicolumn{7}{|l|}{$\overline{\mathcal{M} 5}$} \\
\hline$\overline{\mathbf{R}_{1}}$ & 219 & & $<4$ & -7.22 & 0.0347 & 0.000459 \\
\hline $\mathbf{R}_{\mathbf{2}}$ & 342 & & $>4$ & -210 & 0.909 & 0.0131 \\
\hline
\end{tabular}

Tabela 6.18: Condições e equaçōes das regras obtidas pelos algoritmos Cubist e $\mathcal{M} 5$ para o conjunto de dados Permeability - Cenário 1.

\begin{tabular}{|c|c|c|c|c|c|c|}
\hline \multirow{2}{*}{$\begin{array}{c}\# \\
\text { Regra }\end{array}$} & \multirow{2}{*}{$\begin{array}{l}\# \\
\text { Exs. }\end{array}$} & \multicolumn{2}{|r|}{ Atributos } & \multicolumn{3}{|c|}{ Preditores } \\
\hline & & DEPTH & POROSITY & Constante & POROSITY & DEPTH \\
\hline \multicolumn{7}{|l|}{ Cubist } \\
\hline $\mathrm{R}_{1}$ & 297 & $<=15 \overline{592}$ & $>8,<=13.5$ & -0.13 & $0 . \overline{038}$ & \\
\hline $\mathbf{R}_{\mathbf{2}}$ & 206 & & $<=8$ & -0.01 & 0.015 & \\
\hline $\mathbf{R}_{\mathbf{3}}$ & 79 & $<=15592$ & $>13.5,<=16.4$ & -7.71 & 0.73 & \\
\hline $\mathbf{R}_{\mathbf{4}}$ & 20 & $<=15424$ & $>16.4$ & -192.59 & 1.532 & 0.0111 \\
\hline $\mathbf{R}_{\mathbf{5}}$ & 83 & $>15424$ & $>16.4$ & 103.49 & 2.433 & -0.0086 \\
\hline $\mathrm{R}_{8}$ & 9 & $>15592$ & $>8,<=16.4$ & -5123.86 & 1.239 & 0.3286 \\
\hline \multicolumn{7}{|l|}{$\mathcal{M 5}$} \\
\hline$\vec{R}_{1}$ & $2 \overline{29}$ & & $<=4.0$ & -0.169 & $0 . \overline{0419}$ & \\
\hline $\mathbf{R}_{2}$ & 144 & $<=15600.0$ & $>4.0,<=14.9,<=10.9$ & -0.633 & 0.114 & \\
\hline $\mathbf{R}_{3}$ & 80 & $<=15600.0$ & $>4.0,<=14.9,>10.9,<=13.6$ & 0.147 & 0.122 & \\
\hline R4 & 25 & $<=15600.0$ & $>4.0,<=14.9,>10.9,>13.6$ & 1.67 & 0.122 & \\
\hline $\mathrm{R}_{5}$ & 63 & $>15600.0$ & $>4.0,<=14.9$ & -2830.0 & 0.122 & 0.182 \\
\hline $\mathrm{R}_{8}$ & 153 & & $>4.0,>14.9$ & -466.0 & 1.92 & 0.0287 \\
\hline
\end{tabular}

Tabela 6.19: Condiçōes e equações das regras obtidas pelos algoritmos Cubist e $\mathcal{M} 5$ para o conjunto de dados Permeability - Cenário 2.

\section{PMGRN-455}

O PMGRN-455 é formado por valores de pesagem de um animal em determinados períodos do ano, ou seja, existe uma pesagem do animal aos 120 dias, 240 dias, 365 dias e 455 dias, desde seu nascimento. Anteriormente os animais eram pesados aos 550 dias ao invés de 455 , 


\begin{tabular}{|c|c|c|c|c|c|c|}
\hline \multirow{2}{*}{$\begin{array}{c}\# \\
\text { Regras }\end{array}$} & \multirow{2}{*}{$\begin{array}{l}\# \\
\text { Exs. }\end{array}$} & \multicolumn{2}{|c|}{ Atributos } & \multicolumn{3}{|c|}{ Preditores } \\
\hline & & DEPTH & POROSITY & Constante & POROSITY & DEPTH \\
\hline \multicolumn{7}{|l|}{ Cubist } \\
\hline $\mathrm{R}_{1}$ & 395 & $<=15592$ & $>8,<=13.7$ & -0.08 & $0 . \overline{031}$ & \\
\hline $\mathbf{R}_{2}$ & 239 & & $<=8$ & -0.01 & 0.014 & \\
\hline $\mathbf{R}_{3}$ & 76 & $<=15592$ & $>13.7,<=16.4$ & -5.65 & 0.557 & \\
\hline $\mathbf{R}_{4}$ & 21 & $<=15424$ & $>16.4$ & -161.56 & 1.487 & 0.0091 \\
\hline $\mathbf{R}_{\mathbf{B}}$ & 83 & $>15424$ & $>16.4$ & 126.02 & 2.42 & -0.01 \\
\hline $\mathbf{R}_{6}$ & 9 & $>15592$ & $>8,<=16.4$ & -5177.33 & 1.225 & 0.332 \\
\hline \multicolumn{7}{|l|}{ M5 } \\
\hline $\mathbf{R}_{1}$ & 308 & & $<=4$ & -0.106 & 0.029600 & \\
\hline $\mathrm{R}_{2}$ & 515 & & $>4$ & -9.600 & 1.140 & \\
\hline
\end{tabular}

Tabela 6.20: Condições e equaçōes das regras obtidas pelos algoritmos Cubist e $\mathcal{M} 5$ para o conjunto de dados Permeability - Cenário 3.

mas em análises genéticas feitas pelo grupo de geneticistas da FMRP/USP observou que o peso aos 455 dias é considerado um bom preditor para o peso aos 550 dias. Isto faz com que os criadores apoiados pelo PMGRN vendam seus animais e obtenham lucros com certa antecedência. Neste subconjunto da base de dados do PMGRN, denominado de PMGRN-455 existem 6599 registros de animais, machos e fêmeas. Dessa forma, o PMGRN-455 foi dividido em três conjuntos de dados, PMGRN-455-sexoM, PMGRN-455-sexoF e PMGRN-455-total. Cada um desses conjuntos foi submetido aos algoritmos Cubist e $\mathcal{M} 5$. Os resultados obtidos podem ser observados nas Tabelas 6.21, 6.22 e 6.23. Nessas tabelas podem ser mostradas as condições e as funções matemáticas contidas nas regras obtidas pela utilização dos algoritmos em cada conjunto de dados.

\begin{tabular}{|c|c|c|c|c|c|c|c|c|}
\hline \multirow{2}{*}{$\begin{array}{c}\# \\
\text { Regra }\end{array}$} & \multirow{2}{*}{$\begin{array}{l}\# \\
\text { Exs. }\end{array}$} & \multicolumn{3}{|c|}{$\overline{\text { Atributos }}$} & \multicolumn{4}{|c|}{ Preditores } \\
\hline & & P120 & P240 & P365 & Constunte & P120 & P240 & P365 \\
\hline \multicolumn{9}{|l|}{ Cubist } \\
\hline$\overline{R_{1}}$ & 76 & $<=88$ & & $<=166$ & 47.9 & 0.06 & -0.014 & 0.813 \\
\hline $\mathbf{R}_{2}$ & 149 & $>88,<=100$ & & $<=166$ & 93 & -0.71 & -0.014 & 1.04 \\
\hline $\mathbf{R}_{3}$ & 35 & $<=100$ & $<=138$ & $>166$ & 78.3 & 1.02 & -0.011 & 0.214 \\
\hline $\mathbf{R}_{4}$ & 352 & $<=100$ & $>138$ & $>166,<=204$ & 11.9 & 0.76 & -0.011 & 0.681 \\
\hline $\mathrm{R}_{\text {s }}$ & 437 & $>100$ & $<=168$ & $<=189$ & 12.6 & 0.02 & -0.026 & 1.138 \\
\hline $\mathrm{R}_{6}$ & 170 & $>100$ & $>168,<=178$ & $<=189$ & -25.8 & 0.33 & -0.039 & 1.136 \\
\hline $\mathrm{R}_{\mathbf{7}}$ & 51 & $>100,<=105$ & $<=178$ & $>189,<=204$ & 235.1 & -2.3 & -0.602 & 1.65 \\
\hline $\mathbf{R}_{8}$ & 441 & $>100$ & $>178$ & $<=204$ & 11.7 & 0.38 & -0.157 & 1.005 \\
\hline $\mathbf{R}_{9}$ & 198 & $>105$ & $<=178$ & $>189,<=204$ & 31.7 & 0.07 & -0.041 & 1.031 \\
\hline$R_{10}$ & 133 & $>106$ & $>196$ & $>204,<=216$ & 144.7 & 0.54 & -0.039 & 0.157 \\
\hline$R_{11}$ & 141 & $<106$ & & $>204$ & 95 & 0.05 & -0.024 & 0.669 \\
\hline $\mathrm{R}_{12}$ & 142 & $>106$ & $>184,<=196$ & $>204_{1}<=216$ & 22.4 & 0.47 & -0.036 & 0.808 \\
\hline$R_{13}$ & 264 & $>106$ & $<=184$ & $>204$ & 22.1 & 0.29 & -0.021 & 0.942 \\
\hline$R_{14}$ & 727 & $>106$ & $>184$ & $>216,<=269$ & -8.9 & 0.31 & -0.013 & 1.03 \\
\hline $\mathrm{R}_{15}$ & 67 & & $>184$ & $>269$ & -34.1 & 0.22 & -0.206 & 1.294 \\
\hline \multicolumn{9}{|l|}{ M5 } \\
\hline$\overline{R_{1}}$ & 3383 & & & 7 & 7.24 & 0.496 & -0.244 & 1.06 \\
\hline
\end{tabular}

Tabela 6.21: Condiçōes e equações das regras obtidas utilizando-se os algoritmos Cubist e $\mathcal{M} 5$ no conjunto de dados PMGRN-455-sexoM. 


\begin{tabular}{|c|c|c|c|c|c|c|c|c|}
\hline \multirow{2}{*}{$\begin{array}{c}\# \\
\text { Regra }\end{array}$} & \multirow{2}{*}{$\begin{array}{l}\# \\
\text { Exs. }\end{array}$} & \multicolumn{3}{|c|}{ Atributos } & \multicolumn{4}{|c|}{ Preditores } \\
\hline & & P120 & P240 & P365 & Constunte & P120 & $\mathrm{P} 240$ & P365 \\
\hline \multicolumn{9}{|l|}{ Cubist } \\
\hline$\overline{\mathrm{R}_{1}}$ & 166 & $<=93$ & $<=176$ & & 52.7 & $0.5 \overline{9}$ & -0.535 & 1.024 \\
\hline $\mathbf{R}_{2}$ & 85 & $<=104$ & $>176$ & $<=242$ & 40.2 & 0.09 & -0.079 & 0.937 \\
\hline $\mathbf{R}_{\mathbf{s}}$ & 275 & $>93$ & $<=160$ & $<=242$ & 15.5 & 0.05 & -0.055 & 1.172 \\
\hline $\mathbf{R}_{4}$ & 512 & $>93$ & $>160,<=176$ & $<=242$ & -10.8 & 0.51 & -0.444 & 1.351 \\
\hline $\mathbf{R}_{5}$ & 1319 & $>104$ & $>176$ & $<=242$ & 9.2 & 0.41 & -0.444 & 1.296 \\
\hline$R_{6}$ & 518 & & $<=221$ & $>242$ & -6.9 & 0.01 & -0.009 & 1.224 \\
\hline $\mathrm{R}_{7}$ & 341 & & $>221$ & $>242$ & -33.1 & 0.01 & -0.013 & 1.29 \\
\hline \multicolumn{9}{|l|}{$\overline{\mathcal{M} 5}$} \\
\hline$\overline{\mathrm{R}_{1}}$ & 1515 & & & $<=220$ & 20.6 & $0 . \overline{593}$ & $-\overline{0.557}$ & 1.24 \\
\hline $\mathbf{R}_{2}$ & 1701 & & & $>220$ & 5.71 & 0.27 & $-\mathbf{0 . 3 2 9}$ & 1.29 \\
\hline
\end{tabular}

Tabela 6.22: Condições e equações das regras obtidas utilizando-se os algoritmos Cubist e M5 no conjunto de dados PMGRN-455-sexoF.

\begin{tabular}{|c|c|c|c|c|c|c|c|c|}
\hline \multirow{2}{*}{$\begin{array}{c}\# \\
\text { Regra }\end{array}$} & \multirow{2}{*}{$\begin{array}{l}\# \\
\text { Exx. }\end{array}$} & \multicolumn{3}{|c|}{$\overline{\text { Atributos }}$} & \multicolumn{4}{|c|}{ Preditores } \\
\hline & & P120 & P240 & P365 & Constante & P120 & P240 & P365 \\
\hline \multicolumn{9}{|l|}{ Cubist } \\
\hline$\overline{\mathrm{R}_{1}}$ & 202 & $<=94$ & $<=140$ & & $\overrightarrow{16.6}$ & 0.68 & -0.433 & 1.081 \\
\hline $\mathbf{R}_{2}$ & 185 & $<=90$ & $>140$ & $<=219$ & 14.7 & 0.09 & 0.53 & 0.519 \\
\hline $\mathrm{R}_{3}$ & 163 & $>90,<=94$ & $>140$ & $<=219$ & 48.7 & 0.08 & -0.006 & 0.829 \\
\hline $\mathrm{R}_{4}$ & 1826 & $>94$ & $<=176$ & $<=219$ & 6.8 & 0.43 & -0.401 & 1.252 \\
\hline $\mathrm{R}_{5}$ & 1666 & $>94$ & $>176$ & $<=219$ & 1.3 & 0.51 & -0.337 & 1.163 \\
\hline $\mathrm{R}_{6}$ & $\pi$ & $<=102$ & & $>219$ & 23.5 & 0.05 & -0.047 & 1.022 \\
\hline $\mathrm{R}_{7}$ & 1121 & $>102$ & $<=203$ & $>219$ & 30 & 0.01 & -0.231 & 1.239 \\
\hline $\mathrm{R}_{8}$ & 1365 & $>102$ & $>203$ & $>219$ & -5.2 & 0.25 & -0.26 & 1.276 \\
\hline \multicolumn{9}{|l|}{$\mathcal{M 5}$} \\
\hline $\mathrm{R}_{1}$ & 3696 & & & $<=215$ & 13 & 0.559 & -0.412 & 1.15 \\
\hline $\mathbf{R}_{2}$ & 2903 & & & $>215$ & 2.34 & 0.319 & -0.315 & 1.26 \\
\hline
\end{tabular}

Tabela 6.23: Condiçōes e equações das regras obtidas utilizando-se os algoritmos Cubist e M5 no conjunto de dados PMGRN-455-total.

\subsection{Avaliação do Conhecimento Extraído}

Nesta seção, é descrita uma comparação das regras de regressão a partir de seis conjuntos de dados, utilizando-se os algoritmos Cubist e $\mathcal{M} 5$. O ambiente computacional descrito no Capítulo 5 foi utilizado para obter os resultados das medidas de precisão, compreensibilidade e interessabilidade nesses experimentos. Esses resultados são mostrados a seguir.

\subsubsection{Análise de Precisão}

As taxas de erro calculadas para os conjuntos de dados utilizando o Cubist com 10-fold-crossvalidation pode ser observado na Tabela 6.24. Nesta tabela são apresentados o erro aparente do conjunto de dados, o erro verdadeiro e o número de regras extraídas. Esse processo não pode ser repetido no $\mathcal{M} 5$ devido ao fato que o algoritmo não calcula o erro médio para cada fold de treinamento. 


\begin{tabular}{l|r|r|r}
\hline \hline Conjunto de dados & $\begin{array}{r}\text { Erro } \\
\text { aparente }\end{array}$ & $\begin{array}{r}\text { Erro (CV) } \\
\text { verdadeiro }\end{array}$ & $\begin{array}{r}\text { \# de regras } \\
\text { extraídas }\end{array}$ \\
\hline boston & 1.92 & $2.36 \pm 0.26$ & 7 \\
$\mathrm{cpu}$ & 22.2 & $31.44 \pm 7.87$ & 3 \\
$\mathrm{mpg}$ & 1.81 & $2.15 \pm 0.20$ & 5 \\
Ozone & 2.5 & $3.29 \pm 0.73$ & 7 \\
Permeability-cenario1 & 1.0729 & $1.64 \pm 0.36$ & 14 \\
Permeability-cenario2 & 1.9968 & $2.19 \pm 0.28$ & 6 \\
Permeability-cenario3 & 1.6944 & $1.89 \pm 0.29$ & 6 \\
PMGRN-455-sexoM & 12.6 & $13.09 \pm 0.32$ & 15 \\
PMGRN-455-sexoF & 12.7 & $13.03 \pm 0.77$ & 7 \\
PMGRN-455-Total & 13.2 & $13.36 \pm 0.42$ & 8 \\
\hline
\end{tabular}

Tabela 6.24: Resumo dos resultados do Cubist.

Nesta seção são enfatizadas as precisões em função das taxas de erros com as medidas MSE, NMSE e MADn nas regras de regressão extraídas pelo Cubist e $\mathcal{M} 5$.

Em cada conjunto de dados será mostrada uma tabela contendo os erros de precisão para a predição do atributo-meta, segundo as medidas de desvio padrão (DP), MSE, NMSE e MADn.

\section{CPU}

Na Tabela 6.25 são apresentados os resultados de todas as medidas de precisão das três regras obtidas pelo Cubist e pelo $\mathcal{M} 5$ para o conjunto de dados CPU.

\begin{tabular}{c|cccc}
\hline \hline \# Regra & Médiu \pm DP & MSE & NMSE & MADn \\
\hline Cubist & \multicolumn{5}{c}{} \\
\hline R1 & $156.406 \pm 85.458$ & 4469.168 & 0.612 & 0.105 \\
R2 & $454.414 \pm 265.787$ & 4979.134 & 0.070 & 0.192 \\
R3 & $50.679 \pm 39.019$ & 454.708 & 0.299 & 0.018 \\
\hline M5 & \multicolumn{5}{c}{} \\
\hline R1 & $154.745 \pm 85.489$ & 4809.235 & 0.658 & 0.191 \\
R2 & $456.043 \pm 265.794$ & 6379.007 & 0.090 & 0.043 \\
R3 & $50.446 \pm 39.019$ & 442.924 & 0.291 & 0.028 \\
\hline
\end{tabular}

Tabela 6.25: Medidas de Precisão avaliadas no conjunto de dados CPU.

\section{Boston Housing}

Na Tabela 6.26 podem ser observados os resultados de todas as medidas de precisão das regras obtidas pelo Cubist e pelo $\mathcal{M} 5$ para o conjunto de dados Boston Housing.

Em praticamente todos os conjuntos de dados usados nos experimentos o Cubist gerou um número maior de regras comparado com o $\mathcal{M 5}$. O único conjunto de dados que teve um comportamento diferenciado foi o Boston Housing. Neste caso o Cubist gerou 7 regras enquanto o 


\begin{tabular}{c|cccc}
\hline \hline \# Regra & MédiatDP & MSE & NMSE & MADn \\
\hline Cubist & \multicolumn{5}{c}{} \\
\hline R1 & $25.042 \pm 4.262$ & 5.666 & 0.312 & 0.081 \\
R2 & $13.188 \pm 4.343$ & 5.052 & 0.268 & 0.131 \\
R3 & $18.891 \pm 3.566$ & 6.551 & 0.515 & 0.013 \\
R4 & $28.264 \pm 3.335$ & 8.404 & 0.756 & 0.410 \\
R5 & $34.277 \pm 2.281$ & 7.391 & 1.421 & 0.174 \\
R6 & $40.798 \pm 7.088$ & 14.668 & 0.292 & 0.199 \\
R7 & $46.263 \pm 8.385$ & 19.515 & 0.278 & 0.410 \\
\hline M5 & & & & \\
\hline R1 & $272.238 \pm 244.714$ & 59984.571 & 1.002 & 0.961 \\
R2 & $275.154 \pm 250.257$ & 62713.613 & 1.001 & 0.195 \\
R3 & $278.554 \pm 228.554$ & 52279.192 & 1.001 & 0.976 \\
R4 & $274.134 \pm 251.407$ & 63228.999 & 1.000 & 0.100 \\
R5 & $280.977 \pm 250.305$ & 62669.317 & 1.000 & 0.337 \\
R6 & $273.905 \pm 249.752$ & 62433.409 & 1.001 & 0.334 \\
R7 & $277.154 \pm 250.249$ & 62679.055 & 1.001 & 0.252 \\
R8 & $33.545 \pm 2.772$ & 7.603 & 0.989 & 0.094 \\
R9 & $281.712 \pm 245.072$ & 60088.302 & 1.000 & 0.341 \\
R10 & $286.360 \pm 262.610$ & 68965.239 & 1.000 & 0.500 \\
R11 & $292.440 \pm 247.417$ & 61216.922 & 1.000 & 0.510 \\
R12 & $20.665 \pm 3.110$ & 6.426 & 0.664 & 0.036 \\
R13 & $15.217 \pm 3.762$ & 5.907 & 0.417 & 0.151 \\
R14 & $18.930 \pm 2.521$ & 5.248 & 0.826 & 0.127 \\
R15 & $15.451 \pm 2.382$ & 4.082 & 0.720 & 0.059 \\
R16 & $14.169 \pm 2.230$ & 3.062 & 0.616 & 0.213 \\
R17 & $280.156 \pm 265.454$ & 88268.184 & 1.253 & 0.708 \\
R18 & $247.762 \pm 238.657$ & 79727.928 & 1.400 & 0.613 \\
\hline \hline & & & & \\
\hline & & & & \\
\hline
\end{tabular}

Tabela 6.26: Medidas de Precisão avaliadas no conjunto de dados Boston Housing.

$\mathcal{M} 5$ gerou 18. Deve ser ressaltado que este conjunto de dados apresentava muitos exemplos com valores ausentes e que na etapa de preparação de dados, esses exemplos foram desconsiderados.

\section{MPG}

$\mathrm{Na}$ Tabela 6.27 são apresentados os resultados de todas as medidas de precisão das cinco regras obtidas pelo Cubist e pelo $\mathcal{M} 5$ para o conjunto de dados MPG.

\begin{tabular}{c|cccc}
\hline \hline \# Regra & Média $\pm \bar{D} \bar{P}$ & MSE & NMSE & MADn \\
\hline Cubist & & & & \\
\hline R1 & $24.692 \pm 2.918$ & 4.125 & 0.484 & 0.035 \\
R2 & $16.629 \pm 4.104$ & 5.546 & 0.329 & 0.013 \\
R3 & $29.827 \pm 3.293$ & 4.111 & 0.379 & 0.235 \\
R4 & $29.660 \pm 4.402$ & 7.110 & 0.367 & 0.171 \\
R5 & $36.332 \pm 4.410$ & 15.268 & 0.785 & 0.237 \\
\hline M5 & & & & \\
\hline R1 & $17.963 \pm 1.672$ & 2.166 & 0.775 & 0.183 \\
R2 & $30.683 \pm 6.556$ & 125.208 & 2.913 & 0.052 \\
R3 & $19.938 \pm 1.741$ & 2.204 & 0.727 & 0.038 \\
R4 & $20.846 \pm 7.320$ & 16.260 & 0.303 & 0.222 \\
R5 & $13.825 \pm 2.262$ & 23.525 & 4.598 & 0.029 \\
\hline \hline
\end{tabular}

Tabela 6.27: Medidas de Precisão avaliadas no conjunto de dados MPG. 


\section{Ozone}

Na Tabela 6.28 são apresentados os resultados de todas as medidas de precisão das 7 regras obtidas pelo Cubist e pelas 4 regras obtidas pelo $\mathcal{M} 5$ para o conjunto de dados Ozone.

\begin{tabular}{c|cccc}
\hline \hline \# Regra & MédiutDP & MSE & NMSE & MAD $n$ \\
\hline \multicolumn{1}{c}{ Cubist } \\
\hline R1 & $6.964 \pm 3.129$ & 6.091 & 0.622 & 0.057 \\
R2 & $4.258 \pm 1.486$ & 1.766 & 0.799 & 0.121 \\
R3 & $4.710 \pm 2.946$ & 3.095 & 0.357 & 0.129 \\
R4 & $9.715 \pm 4.292$ & 6.568 & 0.357 & 0.125 \\
R5 & $11.068 \pm 5.016$ & 16.456 & 0.654 & 0.104 \\
R6 & $15.853 \pm 3.989$ & 7.555 & 0.475 & 0.323 \\
R7 & $22.842 \pm 6.188$ & 26.150 & 0.683 & 0.274 \\
\hline $\mathcal{M} 5$ & & & & \\
\hline R1 & $5.452 \pm 2.041$ & 4.572 & 1.097 & 0.141 \\
R2 & $4.795 \pm 2.488$ & 5.049 & 0.815 & 0.279 \\
R3 & $10.237 \pm 4.349$ & 15.214 & 0.804 & 0.105 \\
R4 & $20.453 \pm 6.897$ & 25.943 & 0.545 & 0.134 \\
\hline
\end{tabular}

Tabela 6.28: Medidas de Precisão avaliadas no conjunto de dados Ozone.

\section{Permeability}

Como o conjunto de dados Permeability foi dividido em 3 cenários independentes, nas Tabelas $6.29,6.30$ e 6.31 são apresentados os resultados das medidas de precisão em cada subconjunto ou cenários. Deve ser observado que as regras geradas pelo $\mathcal{M} 5$ possuem uma precisão maior que as regras geradas pelo Cubist.

\begin{tabular}{c|cccc}
\hline \hline \# Regru & Média $\pm \overline{D P}$ & MSE & NMSE & MADn \\
\hline Cubist & & & & \\
\hline R1 & $2.804 \pm 3.406$ & 13.867 & 1.196 & 0.238 \\
R2 & $-0.279 \pm 0.288$ & 0.083 & 1.003 & 0.530 \\
R3 & $0.107 \pm 1.631$ & 2.642 & 0.993 & 0.006 \\
R4 & $1.438 \pm 1.544$ & 2.126 & 0.892 & 0.187 \\
R5 & $3.656 \pm 2.598$ & 4.141 & 0.614 & 0.306 \\
R6 & $6.841 \pm 5.385$ & 10.424 & 0.359 & 0.097 \\
R7 & $7.561 \pm 5.119$ & 15.343 & 0.585 & 0.206 \\
R8 & $11.529 \pm 5.814$ & 21.517 & 0.636 & 0.408 \\
R9 & $12.536 \pm 6.214$ & 10.756 & 0.279 & 0.200 \\
R10 & $12.633 \pm 5.442$ & 33.041 & 1.116 & 0.143 \\
R11 & $14.295 \pm 7.728$ & 40.646 & 0.680 & 0.326 \\
R12 & $16.213 \pm 9.111$ & 72.900 & 0.878 & 0.083 \\
R13 & $23.168 \pm 10.041$ & 123.809 & 1.228 & 0.446 \\
R14 & $25.078 \pm 9.089$ & 74.857 & 0.906 & 0.278 \\
\hline M5 & & & & \\
\hline R1 & $\mathbf{0 . 0 5 9 \pm 0 . 0 7 0}$ & 0.008 & 1.687 & 0.014 \\
R2 & $3.783 \pm 7.291$ & 35.297 & 0.664 & 0.049 \\
\hline \hline
\end{tabular}

Tabela 6.29: Medidas de Precisão avaliadas no conjunto de dados Permeability-cenariol. 


\begin{tabular}{c|cccc}
\hline \hline \# Regra & Média \pm DP & MSE & NMSE & MADn \\
\hline Cubist & & & & \\
\hline R1 & $\mathbf{1 6 . 6 6 9 \pm 1 1 . 8 9 8}$ & 115.338 & 0.815 & 0.301 \\
R2 & $0.030 \pm 0.914$ & 0.831 & 0.994 & 0.004 \\
R3 & $0.278 \pm 1.740$ & 2.992 & 0.989 & 0.011 \\
R4 & $3.256 \pm 6.132$ & 36.424 & 0.969 & 0.175 \\
R5 & $9.372 \pm 5.610$ & 16.806 & 0.534 & 0.104 \\
R6 & $15.257 \pm 11.778$ & 124.103 & 0.895 & 0.091 \\
\hline M5 & & & & \\
\hline R1 & $1.645 \pm 4.856$ & 23.527 & 0.998 & 0.011 \\
R2 & $-0.101 \pm 0.112$ & 0.014 & 1.090 & 0.249 \\
R3 & $\mathbf{0 . 3 3 9 \pm 1 . 4 1 5}$ & 1.979 & 0.988 & 0.017 \\
R4 & $3.408 \pm 5.095$ & 25.773 & 0.993 & 0.032 \\
R5 & $11.125 \pm 3.412$ & 11.722 & 1.007 & 0.852 \\
R6 & $12.412 \pm 10.846$ & 95.508 & 0.812 & 0.240 \\
\hline \hline
\end{tabular}

Tabela 6.30: Medidas de Precisão avaliadas no conjunto de dados Permeability-cenario2.

\begin{tabular}{|c|c|c|c|c|}
\hline \# Regra & Média土DP & $\overline{\mathrm{MSE}}$ & NMSE & $\overline{\mathrm{MADn}}$ \\
\hline \multicolumn{5}{|l|}{$\frac{\pi}{C}$ ubist } \\
\hline R1 & $16.073 \pm 11.929$ & 116.339 & 0.818 & 0.303 \\
\hline R2 & $0.026 \pm 0.794$ & 0.626 & 0.993 & 0.003 \\
\hline R3 & $0.253 \pm 2.085$ & 4.303 & 0.990 & 0.016 \\
\hline $\mathrm{R} 4$ & $2775 \pm 6.088$ & 36.057 & 0.973 & 0.164 \\
\hline R5 & $8.469 \pm 5.695$ & 17.060 & 0.526 & 0.366 \\
\hline R6 & $15.852 \pm 11.736$ & 123.033 & 0.893 & 0.094 \\
\hline \multicolumn{5}{|l|}{$\overline{M 5}$} \\
\hline $\begin{array}{l}\mathrm{R} 1 \\
\mathrm{R} 2\end{array}$ & $\begin{array}{c}-0.061 \pm 0.072 \\
4.450 \pm 8.328\end{array}$ & $\begin{array}{c}0.006 \\
42.966\end{array}$ & $\begin{array}{l}1.092 \\
0.620\end{array}$ & $\begin{array}{l}0.272 \\
0.118\end{array}$ \\
\hline
\end{tabular}

Tabela 6.31: Medidas de Precisão avaliadas no conjunto de dados Permeability-cenario3.

\section{PMGRN-455}

O conjunto PMGRN-455 foi dividido em 3 subconjuntos, 1 subconjunto formado por animais machos, outro pelas fêmeas e o último subconjunto é formado por animais de ambos os sexos. Os resultados obtidos com esses três subconjuntos estão descritos nas Tabelas 6.32, 6.33 e 6.34 .

\begin{tabular}{c|cccc}
\hline \hline \# Regra & Modia \pm DP & MSE & NMSE & MADn \\
\hline Cubist & & & & \\
\hline RI & $221.451 \pm 19.707$ & 294.519 & 0.758 & 0.326 \\
R2 & $171.491 \pm 19.246$ & 230.994 & 0.624 & 0.033 \\
R3 & $185.069 \pm 14.827$ & 145.521 & 0.662 & 0.183 \\
R4 & $202.774 \pm 18.072$ & 253.849 & 0.777 & 0.087 \\
R5 & $205.744 \pm 18.973$ & 285.274 & 0.792 & 0.173 \\
R6 & $206.148 \pm 18.532$ & 193.091 & 0.562 & 0.136 \\
R7 & $210.238 \pm 14.607$ & 155.538 & 0.729 & 0.186 \\
R8 & $223.247 \pm 16.773$ & 218.588 & 0.777 & 0.043 \\
R9 & $234.353 \pm 16.010$ & 240.062 & 0.937 & 0.190 \\
R10 & $238.233 \pm 14.931$ & 198.600 & 0.891 & 0.249 \\
R11 & $239.499 \pm 19.352$ & 338.956 & 0.905 & 0.151 \\
R12 & $241.316 \pm 18.334$ & 323.676 & 0.963 & 0.017 \\
R13 & $256.713 \pm 20.420$ & 288.976 & 0.693 & 0.282 \\
R14 & $274.006 \pm 24.216$ & 331.271 & 0.565 & 0.045 \\
R15 & $316.758 \pm 28.202$ & 429.149 & 0.540 & 0.263 \\
\hline M5 & & & & \\
\hline R1 & $234.233 \pm 36.543$ & 278.753 & 0.209 & 0.013 \\
\hline \hline
\end{tabular}

Tabela 6.32: Medidas de Precisão avaliadas no conjunto de dados PMGRN455-sexoM. 
Observa-se novamente que as regras geradas pelo $\mathcal{M} 5$ são muito mais precisas que as geradas pelo Cubist no conjunto de dados PMGRN455-sexoM.

\begin{tabular}{c|cccc}
\hline \hline \# Regra & MédiatDP & MSE & NMSE & MADn \\
\hline \multicolumn{1}{l}{ Cubist } & & & & \\
\hline R1 & $243.666 \pm 30.691$ & 308.820 & 0.328 & 0.003 \\
R2 & $208.021 \pm 28.590$ & 355.887 & 0.435 & 0.019 \\
R3 & $230.728 \pm 21.104$ & 256.432 & 0.576 & 0.218 \\
R4 & $231.954 \pm 29.332$ & 216.215 & 0.251 & 0.006 \\
R5 & $254.909 \pm 26.593$ & 260.426 & 0.368 & 0.009 \\
R6 & $308.547 \pm 24.385$ & 296.094 & 0.498 & 0.095 \\
R7 & $321.648 \pm 40.912$ & 351.544 & 0.210 & 0.110 \\
\hline M5 & & & & \\
\hline R1 & $232.289 \pm 26.765$ & 271.922 & 0.380 & 0.037 \\
R2 & $293.182 \pm 33.786$ & 315.224 & $\mathbf{0 . 2 7 6}$ & $\mathbf{0 . 0 5 4}$ \\
\hline
\end{tabular}

Tabela 6.33: Medidas de Precisão avaliadas no conjunto de dados PMGRN455-sexoF.

\begin{tabular}{c|cccc}
\hline \hline \# Regra & Média $\pm D P$ & MSE & NMSE & MADn \\
\hline Cubist & & & & \\
\hline R1 & $187.431 \pm 28.137$ & 316.908 & 0.603 & 0.258 \\
R2 & $220.212 \pm 26.540$ & 292.699 & 0.370 & 0.076 \\
R3 & $200.246 \pm 19.806$ & 256.650 & 0.654 & 0.130 \\
R4 & $296.299 \pm 36.118$ & 254.572 & 0.361 & 0.158 \\
R5 & $279.710 \pm 23.469$ & 256.182 & 0.584 & 0.021 \\
R6 & $206.866 \pm 22.932$ & 700.438 & 0.777 & 0.291 \\
R7 & $234.349 \pm 20.953$ & 304.221 & 0.552 & 0.030 \\
R8 & $256.818 \pm 30.024$ & 346.753 & 0.266 & 0.064 \\
\hline M5 & & & & \\
\hline R1 & $221.432 \pm 25.905$ & 260.174 & 0.388 & 0.114 \\
R2 & $285.127 \pm 33.063$ & 349.447 & 0.320 & 0.042 \\
\hline \hline
\end{tabular}

Tabela 6.34: Medidas de Precisão avaliadas no conjunto de dados PMGRN455-total.

As três medidas de precisão: MSE, NMSE e MAD descritas da Tabela 6.25 a 6.34 especificam o erro da predição do atributo-meta para cada conjunto de dados. A MSE consiste na média da diferença ao quadrado entre o valor real e o predito para um atributo-meta. A NMSE consiste da diferença entre o valor real e a média dos valores preditos para o atributo-meta e a MAD consiste da média da diferença (em móduo) de um valor real e predito para o atributo-meta.

\subsubsection{Análise de Compreensibilidade}

Nessa subseção são descritos os resultados obtidos com a avaliação de compreensibilidade das regras de regressão obtidas utilizando o Cubist e o $\mathcal{M} 5$. A compreensibilidade, neste trabalho, é avaliada de acordo com o número de atributos presentes nas condições e a quantidade de atributos presentes nas equações matemáticas que são usadas par a predição.

Um sumário com os resultados obtidos referentes a compreensibilidade das regras de regressão geradas pelo Cubist e pelo $\mathcal{M} 5$ nos conjuntos de dados é apresentado a seguir. 


\begin{tabular}{c|cc}
\hline \hline \# Regra & \# Atributos nus condiçōes & \# Atributos na funçüo \\
\hline Cubist & & \\
\hline R1 & 2 & 6 \\
R2 & 2 & 6 \\
R3 & 1 & 5 \\
\hline $\mathcal{M} 5$ & & \\
\hline R1 & 2 & 5 \\
R2 & 2 & 5 \\
R3 & 1 & 5 \\
\hline \hline
\end{tabular}

Tabela 6.35: Medidas de Compreensibilidade avaliadas no conjunto de dados CPU.

\begin{tabular}{|c|c|c|}
\hline \# Regra & \# Atributos nas condiçōes & \# Atributos na funçũo \\
\hline \multicolumn{3}{|l|}{ Cubist } \\
\hline Rl & 4 & 10 \\
\hline $\mathrm{R} 2$ & 2 & 10 \\
\hline $\mathrm{R3}$ & 3 & 11 \\
\hline $\mathbf{R A}$ & 3 & 11 \\
\hline R5 & 3 & 10 \\
\hline R6 & 2 & 9 \\
\hline R7 & 2 & 9 \\
\hline \multicolumn{3}{|l|}{$\overline{\mathcal{M} 5}$} \\
\hline R1 & 5 & $\overline{11}$ \\
\hline $\mathrm{R} 2$ & 4 & 11 \\
\hline R3 & 5 & 11 \\
\hline $\mathrm{R4}$ & 4 & 11 \\
\hline R5 & 5 & 11 \\
\hline R6 & 6 & 11 \\
\hline R7 & 6 & 11 \\
\hline R8 & 4 & 10 \\
\hline R9 & 5 & 10 \\
\hline R10 & 5 & 10 \\
\hline Rl1 & 3 & 11 \\
\hline R12 & 2 & 11 \\
\hline R13 & 5 & 12 \\
\hline R14 & 5 & 12 \\
\hline R15 & 4 & 11 \\
\hline R16 & 4 & 11 \\
\hline Rl7 & 5 & 11 \\
\hline $\mathrm{R} 18$ & 5 & 11 \\
\hline
\end{tabular}

Tabela 6.36: Medidas de Compreensibilidade avaliadas no conjunto de dados Boston Housing.

\begin{tabular}{|c|c|c|}
\hline \# Regra & \# Atributos nus condiçōes & \# Atributos nu funçǘo \\
\hline \multicolumn{3}{|l|}{ Cubist } \\
\hline$\overline{\mathrm{RI}}$ & $\overline{3}$ & 5 \\
\hline $\mathrm{R} 2$ & I & 2 \\
\hline R3 & 2 & 5 \\
\hline R4 & 3 & 5 \\
\hline R5 & 2 & 5 \\
\hline \multicolumn{3}{|l|}{$\overline{\mathcal{M} 5}$} \\
\hline RI & 4 & 6 \\
\hline $\mathrm{R} 2$ & I & 5 \\
\hline R3 & 3 & 4 \\
\hline R4 & 4 & 6 \\
\hline R5 & 2 & 6 \\
\hline
\end{tabular}

Tabela 6.37: Medidas de Compreensibilidade avaliadas no conjunto de dados MPG.

Para o conjunto de dados Permeability, mantém-se a mesma divisão de cenários, ou seja, Permeability-cenario1, Permeability-cenario2 e Permeability-cenario3. Nas Tabelas 6.39, 6.40 e 6.41 podem ser observadas as medidas para cada subconjunto de dados. 


\begin{tabular}{|c|c|c|}
\hline \# Regra & \# Atributos nas condiçôes & \# Atributos na funçäo \\
\hline \multicolumn{3}{|l|}{ Cubist } \\
\hline $\mathrm{RJ}$ & 4 & $\overline{6}$ \\
\hline $\mathbf{R 2}$ & 2 & 6 \\
\hline R3 & 3 & 6 \\
\hline RA & 2 & 6 \\
\hline R5 & 3 & 6 \\
\hline $\mathrm{R6}$ & 2 & 6 \\
\hline R7 & 3 & 6 \\
\hline \multicolumn{3}{|l|}{ M5 } \\
\hline$\overline{\mathrm{RJ}}$ & $\overline{3}$ & $\overline{6}$ \\
\hline $\mathbf{R 2}$ & 2 & 6 \\
\hline R3 & 3 & 6 \\
\hline RA & 1 & 5 \\
\hline
\end{tabular}

Tabela 6.38: Medidas de Compreensibilidade avaliadas no conjunto de dados Ozone.

\begin{tabular}{c|cc}
\hline \hline \# Regra & \# Atributos nas condiçōes & \# Atributos na funçä́ \\
\hline Cubist & & \\
\hline R1 & 4 & 2 \\
R2 & 1 & 2 \\
R3 & 3 & 2 \\
R4 & 3 & 2 \\
R5 & 3 & 2 \\
R6 & 3 & 2 \\
R7 & 4 & 2 \\
R8 & 3 & 2 \\
R9 & 2 & 2 \\
R10 & 2 & 2 \\
R11 & 3 & 2 \\
RI2 & 3 & 2 \\
R13 & 2 & 2 \\
R14 & 3 & 2 \\
\hline M5 & 3 & 2 \\
\hline R1 & 1 & 2 \\
R2 & 1 & \\
\hline \hline
\end{tabular}

Tabela 6.39: Medidas de Compreensibilidade avaliadas no conjunto de dados PermeabilityCenario1.

\begin{tabular}{|c|c|c|}
\hline \# Regru & \# Atributos nas condições & \# Atributos na funçüo \\
\hline \multicolumn{3}{|c|}{ 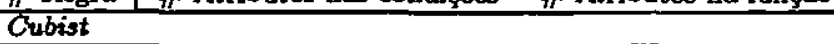 } \\
\hline Rl & 3 & 2 \\
\hline $\mathbf{R 2}$ & 1 & 1 \\
\hline $\mathrm{R} 3$ & 3 & 1 \\
\hline $\mathbf{R A}$ & 3 & 1 \\
\hline R5 & 2 & 2 \\
\hline R6 & 2 & 2 \\
\hline \multicolumn{3}{|l|}{ M5 } \\
\hline$\overline{\mathrm{R} 1}$ & 5 & $I$ \\
\hline R2 & 1 & 1 \\
\hline R3 & 4 & 1 \\
\hline $\mathbf{R A}$ & 5 & 1 \\
\hline R5 & 3 & 2 \\
\hline $\mathrm{R} 6$ & 2 & 2 \\
\hline
\end{tabular}

Tabela 6.40: Medidas de Compreensibilidade avaliadas no conjunto de dados PermeabilityCenario2.

Para o conjunto de dados PMGRN455 foi mantida a divisão entre o sexo do animal (macho ou fêmea) e o conjunto de dados total. Nas Tabelas 6.42, 6.43 e 6.44 podem ser observadas as medidas para os subconjuntos. 


\begin{tabular}{c|cc}
\hline \hline \# Regru & \# Atributos nas condiçöes & \# Atributos na funçäo \\
\hline Cubist & & \\
\hline$\overline{R I}$ & 3 & 2 \\
$\mathrm{R} 2$ & 1 & 1 \\
$\mathrm{R3}$ & 3 & 1 \\
$\mathrm{RA}$ & 3 & 1 \\
$\mathrm{R} 5$ & 2 & 2 \\
$\mathrm{R} 6$ & 2 & 2 \\
\hline $\mathcal{M 5}$ & & \\
\hline $\mathrm{RI}$ & 1 & 1 \\
$\mathrm{R} 2$ & 1 & 1 \\
\hline \hline
\end{tabular}

Tabela 6.41: Medidas de Compreensibilidade avaliadas no conjunto de dados PermeabilityCenario3.

\begin{tabular}{c|cc}
\hline \hline \# Regra & \# Atributo6 nas condiçōes & \# Atributo6 au funçäo \\
\hline Cubist & & \\
\hline RI & 5 & 3 \\
R2 & 2 & 3 \\
R3 & 3 & 3 \\
R4 & 3 & 3 \\
R5 & 4 & 3 \\
R6 & 3 & 3 \\
R7 & 4 & 3 \\
R8 & 3 & 3 \\
R9 & 4 & 3 \\
R10 & 4 & 3 \\
R11 & 2 & 3 \\
R12 & 5 & 3 \\
R13 & 3 & 3 \\
R14 & 4 & 3 \\
R15 & 2 & 3 \\
\hline M5 & & 3 \\
\hline R1 & 1 & \\
\hline \hline
\end{tabular}

Tabela 6.42: Medidas de Compreensibilidade avaliadas no conjunto de dados PMGRN455sexoM.

\begin{tabular}{l|cc}
\hline \hline \# Regrø & \# Atributos nas condiçöes & \# Atributos n\& funçäo \\
\hline Cubist & & \\
\hline RI & 4 & 3 \\
R2 & 2 & 3 \\
R3 & 3 & 3 \\
R4 & 3 & 3 \\
R5 & 3 & 3 \\
R6 & 2 & 3 \\
R7 & 2 & 3 \\
\hline M5 & & \\
\hline R1 & 1 & 3 \\
R2 & 1 & 3 \\
\hline \hline
\end{tabular}

Tabela 6.43: Medidas de Compreensibilidade avaliadas no conjunto de dados PMGRN455sexoF..

Observa-se que as medidas usadas para verificar a compreensibilidade do conhecimento extraído não foram muito úteis, como pode ser observado nas Tabelas de 6.35 a 6.44 . Ressalta-se que o número de atributos da função é praticamente o mesmo para todas as regras e o número de atributos nas condições tem apenas uma pequena variação. Foi verificado também que pelas medidas usadas as regras geradas pelo $\mathcal{M} 5$ são em geral mais compreensíveis que as regras 


\begin{tabular}{c|cc}
\hline \hline \# Regra & \# Atributos nas condiçōes & \# Atributos na função \\
\hline Cubist & & \\
\hline R1 & 4 & 3 \\
R2 & 2 & 3 \\
R3 & 3 & 3 \\
R4 & 3 & 3 \\
R5 & 3 & 3 \\
R6 & 2 & 3 \\
R7 & 3 & 3 \\
R8 & 3 & 3 \\
\hline M5 & & \\
\hline R1 & 1 & 3 \\
R2 & 1 & 3 \\
\hline \hline
\end{tabular}

Tabela 6.44: Medidas de Compreensibilidade avaliadas no conjunto de dados PMGRN455-total.

geradas pelo Cubist. Visto que nas tabelas anteriores pode ser observada que a quantidade de atributos nas condições e funções das regras geradas pelo $\mathcal{M} 5$ quase sempre é menor que as existentes nas do Cubist.

\subsubsection{Análise de Interessabilidade}

Nessa subseção são descritas as análises de interessabilidade obtidas mediante a utilização das medidas de GanhoMAD e $Q$ descritas na seção 3.5 on page 23. Em todos os experimentos realizados para calcular a medida de interessabilidade $Q$ foi utilizada a constante $w_{\text {ganho }}$ calculado usando $w_{1}=0.75$ e $w_{2}=0.9$. Sendo que nesses experimentos os resultados são detalhados por cada conjunto de dados, a seguir.

\section{CPU}

Na Tabeia 6.45 podem ser observados os resultados das medidas de interessabilidade do conjunto de dados CPU. Segundo a medida $Q$, a regra $R 1$ do Cubist é a mais interessante, enquanto que para o $\mathcal{M} 5$ é a regra $\mathrm{R} 3$ a de maior interesse.

\begin{tabular}{|c|c|c|}
\hline \# Rogra & GanhoMAD & $\overline{\mathbf{Q}}$ \\
\hline \multicolumn{3}{|l|}{ Cubist } \\
\hline$\overline{\mathbf{R}} \mathbf{1}$ & 0.966 & 0.966 \\
\hline R2 & 0.495 & 0.831 \\
\hline R3 & 0.763 & 0.712 \\
\hline RA & 0.926 & 0.896 \\
\hline R5 & 0.754 & 0.726 \\
\hline \multicolumn{3}{|l|}{$\overline{\mathcal{M} 5}$} \\
\hline$\overline{\mathrm{RI}}$ & 0.809 & 0.809 \\
\hline R2 & 0.946 & 0.962 \\
\hline R3 & 0.966 & 1.690 \\
\hline
\end{tabular}

Tabela 6.45: Medidas de Interessabilidade avaliadas no conjunto de dados CPU. 


\section{Boston Housing}

Na Tabela 6.46 pode ser observado os resultados das medidas de interessabilidade do conjunto de dados Boston Housing. Segundo a medida Q, as regras R3 do Cubist e $\mathcal{M} 5$ são as mais interessantes, apesar que o valor $Q$ da regra $R 3 \mathcal{M} 5$ possuir um valor muito alto. Esse valor alto se justifica pela regra possuir muitos exemplos que conseqüentemente contribuem para que o erro dela seja mínimo.

\begin{tabular}{|c|c|c|}
\hline \# Regra & GamoMAD & $\overline{\overline{\mathbf{Q}}}$ \\
\hline \multicolumn{3}{|l|}{ Cubist } \\
\hline$\overline{\mathbf{R}}$ & 0.919 & $\overline{0.919}$ \\
\hline $\mathbf{R} 2$ & 0.858 & 0.829 \\
\hline R3 & 0.986 & 1.006 \\
\hline R4 & a.554 & 0.467 \\
\hline $\mathrm{RS}$ & 0.811 & 0.713 \\
\hline R6 & 0.783 & 0.722 \\
\hline R7 & 0.554 & 0.468 \\
\hline \multicolumn{3}{|l|}{ M5 } \\
\hline$\overline{\mathrm{R} 1}$ & 0.039 & 0.039 \\
\hline $\mathrm{R} 2$ & $\mathbf{3 . 9 7 3}$ & 5.873 \\
\hline R3 & -23.823 & 72.360 \\
\hline R4 & -1.544 & 10.759 \\
\hline R5 & -7.577 & 9.862 \\
\hline $\mathrm{R} 6$ & -7.503 & 6.026 \\
\hline R7 & -5.419 & 6.718 \\
\hline $\mathrm{R} 8$ & -1.393 & 3.836 \\
\hline R9 & -7.679 & 6.824 \\
\hline Rio & -11.723 & 13.160 \\
\hline Ri1 & -11.972 & 32.979 \\
\hline R12 & 0.086 & 10.501 \\
\hline R13 & -2.840 & 1.333 \\
\hline RI4 & -2.236 & 6.098 \\
\hline R15 & -0.513 & 3.688 \\
\hline $\mathrm{R}_{16}$ & -4.421 & 8.443 \\
\hline R17 & -17.001 & 45.541 \\
\hline R18 & -14.592 & 48.636 \\
\hline
\end{tabular}

Tabela 6.46: Medidas de Interessabilidade avaliadas no conjunto de dados Boston Housing.

\section{MPG}

Na Tabela 6.47 são apresentados os resultados de interessabilidade para o conjunto de dados MPG. A partir desses resultados, verifica-se que as regras $\mathrm{R} 1$ e $\mathrm{R} 2$ geradas pelo Cubist e pelo $\mathcal{M} 5$, respectivamente, são as mais interessantes.

\section{Ozone}

Na Tabela 6.48 são apresentados os resultados da avaliação de GanhoMAD e Q no conjunto de dados Ozone. A partir desses resultados, observa-se que as regras R1 e R3 geradas pelo Cubist e $\mathcal{M} 5$, respectivamente, são as mais interessantes. 


\begin{tabular}{|c|c|c|}
\hline \# Regru & GanhoMAD & $\overline{\mathbf{Q}}$ \\
\hline \multicolumn{3}{|l|}{ Cubist } \\
\hline$\overline{\mathbf{R} 1}$ & 0.966 & 0.966 \\
\hline $\mathrm{R} 2$ & 0.495 & 0.831 \\
\hline R3 & 0.763 & 0.712 \\
\hline RA & 0.926 & 0.896 \\
\hline R5 & 0.754 & 0.726 \\
\hline \multicolumn{3}{|l|}{$\overline{\mathcal{M} 5}$} \\
\hline R1 & 0.817 & 0.817 \\
\hline R2 & 0.936 & 1.895 \\
\hline $\mathrm{R3}$ & 0.953 & 1.054 \\
\hline RA & 0.729 & 0.678 \\
\hline R5 & 0.965 & 1.273 \\
\hline
\end{tabular}

Tabela 6.47: Medidas de Interessabilidade avaliadas no conjunto de dados MPG.

\begin{tabular}{|c|c|c|}
\hline \# Regra & GanhoMAD & $\overline{\mathbf{Q}}$ \\
\hline \multicolumn{3}{|l|}{ Cubist } \\
\hline $\mathbf{R} 1$ & $\overline{0.944}$ & $0 . \overline{944}$ \\
\hline $\mathbf{R 2}$ & 0.871 & 0.838 \\
\hline R3 & 0.863 & 0.799 \\
\hline RA & 0.868 & 0.782 \\
\hline R5 & 0.890 & 0.869 \\
\hline R6 & 0.658 & 0.590 \\
\hline R7 & 0.709 & 0.737 \\
\hline \multicolumn{3}{|l|}{ M5 } \\
\hline$\overline{\mathbf{R} 1}$ & $\overline{0.859}$ & $0 . \overline{859}$ \\
\hline $\mathbf{R} 2$ & 0.675 & 0.986 \\
\hline R3 & 0.877 & 1.117 \\
\hline RA & 0.844 & 1.110 \\
\hline
\end{tabular}

Tabela 6.48: Medidas de Interessabilidade avaliadas no conjunto de dados Ozone.

\section{Permeability}

Nas Tabelas 6.49, 6.50 e 6.51 são apresentados os valores obtidos ao aplicar as medidas de interessabilidade no conjunto de dados Permeability-cenario1, Permeability-cenario2 e PermeabilityCenario3 propostos por [Rogers et al., 1995]. Segundo a medida Q, para o cenário 1, a regra $\mathrm{R} 2$ gerada pelo Cubist é a mais interessante, enquanto a regra $\mathrm{R} 2$ gerada pelo $\mathcal{M} 5$ é a mais interessante.

Para o cenário 2, as regras mais interessantes são R2 e R3 gerados pelos Cubist e $\mathcal{M} 5$, respectivamente. Enquanto para o cenário 3, as regras mais interessantes são R2 e R2 geradas pelo Cubist e $\mathcal{M} 5$, respectivamente.

\section{PMGRN-455}

O conjunto de dados PMGRN455 divididos em três subconjuntos foram utilizados para calcular as medidas de avaliação de interessabilidade GanhoMAD e Q. Os resultados obtidos para cada subconjunto podem ser observados nas Tabelas $6.52,6.53$ e 6.54. Para o primeiro subconjunto 


\begin{tabular}{|c|c|c|}
\hline \# Regru & GanhoMAD & $\bar{Q}$ \\
\hline \multicolumn{3}{|l|}{ Cubist } \\
\hline$\overline{\mathbf{R I}}$ & 0.762 & 0.762 \\
\hline $\mathbf{R 2}$ & 0.304 & 1.911 \\
\hline R3 & 0.992 & 1.743 \\
\hline R4 & 0.755 & 0.846 \\
\hline R5 & 0.598 & 0.622 \\
\hline $\mathbf{R 6}$ & 0.873 & 0.868 \\
\hline R7 & 0.729 & 0.715 \\
\hline R8 & 0.465 & 0.418 \\
\hline $\mathbf{R} 9$ & 0.738 & 0.664 \\
\hline R10 & 0.813 & 0.746 \\
\hline R11 & 0.572 & 0.540 \\
\hline R12 & 0.891 & 0.845 \\
\hline R13 & 0.415 & 0.377 \\
\hline R14 & 0.636 & 0.595 \\
\hline \multicolumn{3}{|l|}{$\overline{M 5}$} \\
\hline R1 & 0.986 & 0.986 \\
\hline $\mathbf{R 2}$ & 0.951 & 1.016 \\
\hline
\end{tabular}

Tabela 6.49: Medidas de Interessabilidade avaliadas no conjunto de dados Permeabilitycenario1.

\begin{tabular}{c|cc}
\hline \hline \# Regra & GanhoMAD & Q \\
\hline Cubist & & \\
\hline R1 & 0.699 & 0.699 \\
R2 & 0.995 & 4.221 \\
R3 & 0.985 & 3.225 \\
R4 & 0.750 & 1.854 \\
R5 & 0.851 & 1.019 \\
R6 & 0.870 & 1.868 \\
\hline $\mathcal{M 5}$ & & \\
\hline R1 & 0.989 & 0.989 \\
R2 & 0.748 & 1.000 \\
R3 & 0.983 & 1.101 \\
R4 & 0.967 & 0.902 \\
R5 & 0.139 & 0.117 \\
R6 & 0.758 & 0.886 \\
\hline \hline
\end{tabular}

Tabela 6.50: Medidas de Interessabilidade avaliadas no conjunto de dados Permeabilitycenario2.

\begin{tabular}{c|cc}
\hline \hline \# Regra & GanhoMAD & Q \\
\hline Cubist & & \\
\hline R1 & 0.697 & 0.697 \\
R2 & 0.996 & 5.310 \\
R3 & 0.977 & 3.624 \\
R4 & 0.765 & 1.804 \\
R5 & 0.475 & 0.807 \\
R6 & 0.866 & 1.870 \\
\hline M5 & & \\
\hline R1 & 0.728 & 0.728 \\
R2 & 0.838 & 0.941 \\
\hline \hline
\end{tabular}

Tabela 6.51: Medidas de Interessabilidade avaliadas no conjunto de dados Permeabilitycenario3.

PMGRN455-sexoM foram determinadas as regras R14 e R1 geradas pelo Cubist e $\mathcal{M} 5$, sendo as mais interessantes.

Para o subconjunto PMGRN455-sexoF foram determinadas que as regras R5 e R1 geradas pelo 


\begin{tabular}{|c|c|c|}
\hline \# Regru & GanhoMAD & $\overline{\mathbf{Q}}$ \\
\hline \multicolumn{3}{|l|}{ Cubist } \\
\hline$\overline{\mathrm{R} 1}$ & 0.674 & 0.674 \\
\hline R2 & 0.950 & 1.008 \\
\hline R3 & 0.729 & 1.037 \\
\hline R4 & 0.871 & 0.849 \\
\hline R5 & 0.743 & 1.596 \\
\hline R6 & 0.799 & 1.810 \\
\hline R7 & 0.724 & 1.093 \\
\hline $\mathrm{R} 8$ & 0.936 & 1.781 \\
\hline $\mathbf{R} 9$ & 0.718 & 1.168 \\
\hline R10 & 0.630 & 0.938 \\
\hline R11 & 0.776 & 1.042 \\
\hline R12 & 0.975 & 1.163 \\
\hline R13 & 0.582 & 1.330 \\
\hline R14 & 0.934 & 2.398 \\
\hline R15 & 0.610 & 0.722 \\
\hline \multicolumn{3}{|l|}{$\mathcal{M 5}$} \\
\hline$\overline{\mathrm{R} 1}$ & 0.987 & 0.987 \\
\hline
\end{tabular}

Tabela 6.52: Medidas de Interessabilidade avaliadas no conjunto de dados PMGRN455-sexoM.

Cubist e $\mathcal{M} 5$ respectivamente como sendo as mais interessantes.

\begin{tabular}{c|cc}
\hline \hline \# Regra & GanhoMAD & Q \\
\hline Cubist & & \\
\hline R1 & 0.998 & 0.998 \\
R2 & 0.981 & 0.913 \\
R3 & 0.782 & 0.700 \\
R4 & 0.994 & 0.948 \\
R5 & 0.991 & 1.152 \\
R6 & 0.905 & 0.917 \\
R7 & 0.890 & 0.864 \\
\hline M5 & & \\
\hline R1 & 0.963 & 0.963 \\
R2 & 0.944 & 0.959 \\
\hline \hline
\end{tabular}

Tabela 6.53: Medidas de Interessabilidade avaliadas no conjunto de dados PMGRN455-sexoF.

Para o subconjunto PMGRN455-total foram determinadas as regras R4 e R2 geradas pelo Cubist e $\mathcal{M} 5$ respectivamente, como sendo as mais interessantes.

\begin{tabular}{|c|c|c|}
\hline \# Regra & GanhoMAD & $\overline{\mathbf{Q}}$ \\
\hline \multicolumn{3}{|l|}{ Cubist } \\
\hline$\overline{\mathrm{R} 1}$ & 0.762 & 0.762 \\
\hline R2 & 0.900 & 0.939 \\
\hline R3 & 0.829 & 0.868 \\
\hline R4 & 0.793 & 2.157 \\
\hline R5 & 0.973 & 1.935 \\
\hline R6 & 0.619 & 0.590 \\
\hline $\mathrm{R} 7$ & 0.961 & 1.587 \\
\hline $\mathrm{n} 8$ & 0.917 & 1.756 \\
\hline \multicolumn{3}{|l|}{$\overline{M 5}$} \\
\hline$\overline{\mathrm{R} 1}$ & 0.886 & 0.886 \\
\hline R2 & 0.953 & 0.931 \\
\hline
\end{tabular}

Tabela 6.54: Medidas de Interessabilidade avaliadas no conjunto de dados PMGRN455-total. 


\subsubsection{Síntese das Medidas Avaliadas}

Identificar as regras de maior interesse para o especialista do domínio não é uma tarefa fácil, pois nem sempre as regras consideradas precisas, compreensíveis e interessantes satisfazem o critério do especialista. Sendo necessário comparar todas as medidas de avaliação para posteriormente validar com o especialista do domínio.

Nesta subseção é apresentada a síntese de todas as medidas de avaliação do conhecimento extraído para cada um dos conjuntos de dados avaliados pelo $\mathcal{R} \mathcal{R}_{\text {Evaluation. }}$

Para o primeiro conjunto de dados CPU, pode ser observado que os resultados mostrados na Tabela 6.55 indicam que a regra R3 gerada pelo Cubist é a regra de maior interesse dentre todas as extraídas pelo Cubist. Já para o $\mathcal{M} 5$ a regra. $\mathrm{R3}$, apesar de ser a mais interessante, não é a mais precisa de todas as regras extraídas pelo $\mathcal{M} 5$, segundo a medida NMSE.

\begin{tabular}{|c|c|c|c|c|c|c|c|c|}
\hline \multirow{4}{*}{$\begin{array}{r}\begin{array}{r}\# \\
\text { Regru }\end{array} \\
\text { Cubist } \\
\overline{R 1}\end{array}$} & \multicolumn{4}{|c|}{$\overline{\overline{\text { Precisão }}}$} & \multicolumn{2}{|c|}{ Compreensibilidade } & \multicolumn{2}{|c|}{ lnteressabilidade } \\
\hline & Média \pm DP & MSE & NMSE & MADn & \# Cond. & \# Pred. & Ganho & $\mathbf{Q}$ \\
\hline & \multicolumn{8}{|l|}{ Howa $\perp$ LI } \\
\hline & $156.406 \pm 85.458$ & 4469.168 & 0.612 & 0.105 & 2 & 6 & 0.895 & 0.895 \\
\hline R2 & $454.414 \pm 265.787$ & 4979.134 & 0.070 & 0.192 & 2 & 6 & 0.786 & 0.827 \\
\hline R3 & $50.679 \pm 39.019$ & 454.708 & 0.299 & 0.018 & 1 & 5 & 0.980 & 1.688 \\
\hline \multicolumn{9}{|l|}{$\mathcal{M 5}$} \\
\hline$\overline{\mathrm{Rl}}$ & $154.745 \pm 85.489$ & 4809.235 & 0.658 & 0.191 & 2 & $\overline{5}$ & 0.809 & $\overline{0.809}$ \\
\hline $\mathbf{R 2}$ & $456.043 \pm 265.794$ & 6379.007 & 0.090 & 0.043 & 2 & 5 & 0.946 & 0.962 \\
\hline R3 & $50.446 \pm 39.019$ & 442.924 & 0.291 & 0.028 & 1 & 5 & 0.966 & 1.690 \\
\hline
\end{tabular}

Tabela 6.55: Síntese das medidas avaliadas para o conjunto de dados CPU.

Para o conjunto de dados Boston Housing, cujos valores estão representandos na Tabela 6.56, a regra mais interessante gerada pelo Cubist é a regra $R 3$, apesar do valor da medida NMSE ser um dos mais altos das regras extraídas pelo Cubist. Já para as regras geradas pelo $\mathcal{M}^{2}$ pode ser observada que a regra mais interessante $R 3$ possui $Q$ igual 72.360 o que é muito alto, além disso o valor da MSE para essa regra é muito alto e a NMSE possui um valor próximo a 1, ou seja, a R3 tende a predizer o valor médio e seus erros são discrepantes com os valores reais contidos no conjunto de dados.

Para o conjunto de dados MPG cujos valores de precisão, compreensibilidade e interessabilidade estāo representados na Tabela 6.57. possuem as regras mais interessantes R1 e R3 geradas pelos algoritmos Cubist e $\mathcal{M} 5$ respectivamente. Apesar que ambas possuem um desvio padrão consideravelmente similar ao das outras regras.

\footnotetext{
${ }^{2} \mathrm{O}$ algoritmo $\mathcal{M} 5$ gerou 18 regras em comparação com as 7 regras geradas pelo Cubist.
} 


\begin{tabular}{|c|c|c|c|c|c|c|c|c|}
\hline \multirow{2}{*}{$\begin{array}{r}\# \\
\text { Regru } \\
\end{array}$} & \multicolumn{4}{|c|}{ Precisäo } & \multicolumn{2}{|c|}{ Compreensibilidade } & \multicolumn{2}{|c|}{ Interessubilidade } \\
\hline & Média \pm DP & MSE & NMSE & MADn & \# Cond. & \# Pred. & Gunho & $\mathbf{Q}$ \\
\hline \multicolumn{9}{|l|}{ Cubist } \\
\hline$\overline{\mathrm{R} 1}$ & $25.042 \pm 4.262$ & 5.666 & 0.312 & 0.081 & 4 & 10 & 0.919 & 0.919 \\
\hline $\mathrm{R2}$ & $13.188 \pm 4.343$ & 5.052 & 0.268 & 0.131 & 2 & 10 & 0.858 & 0.829 \\
\hline $\mathbf{R 3}$ & $18.891 \pm 3.566$ & 6.551 & 0.515 & 0.013 & 3 & 11 & 0.986 & 1.006 \\
\hline R4 & $28.264 \pm 3.335$ & 8.404 & 0.756 & 0.410 & 3 & 11 & 0.554 & 0.467 \\
\hline R5 & $34.277 \pm 2.281$ & 7.391 & 1.421 & 0.174 & 3 & 10 & 0.811 & 0.713 \\
\hline $\mathbf{R 6}$ & $40.798 \pm 7.088$ & 14.668 & 0.292 & 0.199 & 2 & 9 & 0.783 & 0.722 \\
\hline R7 & $46.263 \pm 8.385$ & 19.515 & 0.278 & 0.410 & 2 & 9 & 0.554 & 0.468 \\
\hline \multicolumn{9}{|l|}{$\mathcal{M} 5$} \\
\hline$\overline{\mathrm{R} 1}$ & $272.238 \pm 244.714$ & 59984.571 & 1.002 & 0.961 & 5 & 11 & 0.039 & $\overline{0.039}$ \\
\hline $\mathrm{R} 2$ & $275.154 \pm 250.257$ & 62713.613 & 1.001 & 0.195 & 4 & 11 & -3.973 & 5.873 \\
\hline $\mathbf{R 3}$ & $278.554 \pm 228.554$ & 52279.192 & 1.001 & 0.976 & 5 & 11 & -23.823 & 72.360 \\
\hline R4 & $274.134 \pm 251.407$ & 63228.999 & 1.000 & 0.100 & 4 & 11 & -1.544 & 10.759 \\
\hline R5 & $280.977 \pm 250.305$ & 62669.317 & 1.000 & 0.337 & 5 & 11 & -7.577 & 9.862 \\
\hline R6 & $273.905 \pm 249.752$ & 62433.409 & 1.001 & 0.334 & 6 & 11 & -7.503 & 6.026 \\
\hline R7 & $277.154 \pm 250.249$ & 62679.055 & 1.001 & 0.252 & 6 & 11 & -5.419 & 6.718 \\
\hline $\mathrm{R} 8$ & $33.545 \pm 2.772$ & 7.603 & 0.989 & 0.094 & 4 & 10 & -1.393 & 3.836 \\
\hline R9 & $281.712 \pm 245.072$ & 60088.302 & 1.000 & 0.341 & 5 & 10 & -7.679 & 6.824 \\
\hline R10 & $288.360 \pm 262.610$ & 68965.239 & 1.000 & 0.500 & 5 & 10 & -11.723 & 13.160 \\
\hline R11 & $292.440 \pm 247.417$ & 61216.922 & 1.000 & 0.510 & 3 & 11 & -11.972 & 32.979 \\
\hline R12 & $20.665 \pm 3.110$ & 6.426 & 0.664 & 0.036 & 2 & 11 & 0.086 & 10.501 \\
\hline R13 & $15.217 \pm 3.762$ & 5.907 & 0.417 & 0.151 & 5 & 12 & -2.840 & 1.333 \\
\hline R14 & $18.930 \pm 2.521$ & 5.248 & 0.826 & 0.127 & 5 & 12 & -2.236 & 6.098 \\
\hline R15 & $15.451 \pm 2.382$ & 4.082 & 0.720 & 0.059 & 4 & 11 & -0.513 & 3.688 \\
\hline R16 & $14.169 \pm 2.230$ & 3.062 & 0.616 & 0.213 & 4 & 11 & -4.421 & 8.443 \\
\hline R17 & $280.156 \pm 265.454$ & 88268.184 & 1.253 & 0.708 & 5 & 11 & -17.001 & 45.541 \\
\hline R18 & $247.762 \pm 238.657$ & 79727.928 & 1.400 & 0.613 & 5 & 11 & -14.592 & 48.636 \\
\hline
\end{tabular}

Tabela 6.56: Síntese das medidas avaliadas para o conjunto de dados Boston Housing.

\begin{tabular}{|c|c|c|c|c|c|c|c|c|}
\hline \multirow{2}{*}{$\begin{array}{r}\# \\
\text { Regra }\end{array}$} & \multicolumn{4}{|c|}{$\overline{\text { Precista }}$} & \multicolumn{2}{|c|}{ Compreensibilidade } & \multicolumn{2}{|c|}{ Interessabilidade } \\
\hline & Média $\pm \mathrm{DP}$ & MSE & NMSE & MADn & \# Cond & \# Pred. & Ganho & Q \\
\hline \multicolumn{9}{|l|}{ Cubist } \\
\hline $\mathrm{R} 1$ & $24.692 \pm 2.918$ & 4.125 & 0.484 & 0.035 & 3 & $\overline{5}$ & 0.966 & 0.966 \\
\hline R2 & $16.629 \pm 4.104$ & 5.546 & 0.329 & 0.013 & 1 & 2 & 0.987 & 1.118 \\
\hline R3 & $29.827 \pm 3.293$ & 4.111 & 0.379 & 0.235 & 2 & 5 & 0.757 & 0.704 \\
\hline $\mathrm{R} 4$ & $29.660 \pm 4.402$ & 7.110 & 0.367 & 0.171 & 3 & 5 & 0.823 & 0.796 \\
\hline R5 & $36.332 \pm 4.410$ & 15.268 & 0.785 & 0.237 & 2 & 5 & 0.754 & 0.722 \\
\hline \multicolumn{9}{|l|}{$\bar{M} 5$} \\
\hline RI & $17.963 \pm 1.672$ & 2.166 & 0.775 & 0.183 & 4 & 6 & $\overline{0.817}$ & 0.817 \\
\hline R2 & $30.683 \pm 6.556$ & 125.208 & 2.913 & 0.052 & 1 & $\mathbf{5}$ & 0.936 & 1.895 \\
\hline $\mathbf{R 3}$ & $19.938 \pm 1.741$ & 2.204 & 0.727 & 0.038 & 3 & 4 & 0.953 & 1.054 \\
\hline R4 & $20.846 \pm 7.320$ & 16.260 & 0.303 & 0.222 & 4 & 6 & 0.729 & 0.678 \\
\hline R5 & $13.825 \pm 2.262$ & 23.525 & 4.598 & 0.029 & 2 & 6 & 0.965 & 1.273 \\
\hline
\end{tabular}

Tabela 6.57: Síntese das medidas avaliadas para o conjunto de dados MPG.

Para o conjunto de dados Ozone pode ser observado na Tabela 6.58 que as regras R1 e R3 do Cubist e do $\mathcal{M} 5$, respectivamente, são as regras mais interessantes segundo as medidas de interessabilidade. 


\begin{tabular}{|c|c|c|c|c|c|c|c|c|}
\hline \multirow{2}{*}{$\begin{array}{r}\# \\
\text { Regra }\end{array}$} & \multicolumn{4}{|c|}{ Precisüo } & \multicolumn{2}{|c|}{ Compreensibilidade } & \multicolumn{2}{|c|}{ Interessabilidade } \\
\hline & Médin 土 DP & MSE & NMSE & MADn & \# Cond. & \# Pred. & Ganho & Q \\
\hline \multicolumn{9}{|l|}{ Cubist } \\
\hline$\overline{R I}$ & $6.964 \pm 3.129$ & 6.091 & 0.622 & 0.057 & 4 & 6 & 0.944 & $\overline{0.944}$ \\
\hline $\mathbf{R 2}$ & $4.258 \pm 1.486$ & 1.766 & 0.799 & 0.121 & 2 & 6 & $0.87 I$ & 0.838 \\
\hline $\mathbf{R 3}$ & $4.710+2.946$ & 3.095 & 0.357 & 0.129 & $\mathbf{3}$ & 6 & 0.863 & 0.799 \\
\hline R4 & $9.775 \pm 4.292$ & 6.568 & 0.357 & 0.125 & 2 & 6 & 0.868 & 0.782 \\
\hline R5 & $11.068 \pm 5.016$ & 16.456 & 0.654 & 0.104 & 3 & 6 & 0.890 & 0.869 \\
\hline $\mathbf{R 6}$ & $15.853 \pm 3.989$ & 7.555 & 0.475 & 0.323 & 2 & 6 & 0.658 & 0.590 \\
\hline R7 & $22.842 \pm 6.188$ & 26.150 & 0.683 & 0.274 & 3 & 6 & 0.709 & 0.737 \\
\hline \multicolumn{9}{|l|}{$\overline{\mathrm{M5}}$} \\
\hline $\mathbf{R 1}$ & $5.452 \pm 2.041$ & 4.572 & 1.097 & 0.141 & $\overline{3}$ & 6 & 0.859 & $\overline{0.859}$ \\
\hline R2 & $4.795 \pm 2.488$ & 5.049 & 0.815 & 0.279 & 2 & 6 & 0.675 & 0.986 \\
\hline $\mathbf{R 3}$ & $10.237 \pm 4.349$ & 15.214 & 0.804 & 0.105 & 3 & 6 & 0.877 & 1.117 \\
\hline R4 & $20.453 \pm 6.897$ & 25.943 & 0.545 & 0.134 & I & 5 & 0.844 & 1.110 \\
\hline
\end{tabular}

Tabela 6.58: Síntese das medidas avaliadas para o conjunto de dados Ozone.

Para o conjunto de dados Permeability, que foi dividido em 3 partes. A síntese das avaliações feitas a cada subconjunto estão em tabelas separadas. As informações para o conjunto de dados Permeability-cenariol encontra-se na Tabela 6.59, enquanto as informações para o conjunto Permeability-cenario2 e Permeability-cenario3 encontram-se nas Tabelas 6.60 e 6.61. De acordo com as medidas observadas na Tabela 6.59 as regras $\mathrm{R} 2$ ambas do Cubișt e $\mathcal{M} 5$ são as mais interessantes. Para a análise do conjunto Permeability-cenario2 Permeability-cenario3 foram verificadas somente as regras mais interessantes, tais como a R2 e R3 geradas pelo Cubist e $\mathcal{M} 5$ respectivamente para o cenário2 e para o cenário3 as regras $\mathrm{R} 2$ e $\mathrm{R} 2$ para geradas pelos algoritmos Cubist e $\mathcal{M} 5$ respectivamente.

\begin{tabular}{|c|c|c|c|c|c|c|c|c|}
\hline \multirow{3}{*}{$\begin{array}{r}\# \\
\text { Regrs } \\
\text { Cubist } \\
\end{array}$} & \multicolumn{4}{|c|}{ Precisǘ } & \multicolumn{2}{|c|}{ Compreensibilidade } & \multicolumn{2}{|c|}{ Interessabilidade } \\
\hline & Média $\pm D P$ & MSE & NMSE & MADn & \# Cond. & \# Pred. & Ganho & \\
\hline & \multicolumn{8}{|l|}{ 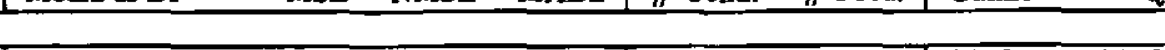 } \\
\hline $\mathbf{R 1}$ & $2.804 \pm 3.406$ & 13.867 & 1.196 & 0.238 & 4 & $\overline{2}$ & 0.762 & 0.762 \\
\hline $\mathbf{R 2}$ & $-0.279 \pm 0.288$ & 0.083 & $\mathrm{I} .003$ & 0.530 & 1 & 2 & 0.304 & 1.911 \\
\hline $\mathbf{R 3}$ & $0.107 \pm 1.631$ & 2.642 & 0.993 & 0.006 & 3 & 2 & 0.992 & 1.743 \\
\hline $\mathbf{R A}$ & $1.438 \pm 1.544$ & 2.126 & 0.892 & 0.187 & 3 & 2 & 0.755 & 0.846 \\
\hline R5 & $3.656 \pm 2.598$ & 4.141 & 0.614 & 0.306 & 3 & 2 & 0.598 & 0.622 \\
\hline R6 & $6.841 \pm 5.385$ & 10.424 & 0.359 & 0.097 & 3 & 2 & 0.873 & 0.868 \\
\hline R7 & $7.561 \pm 5.119$ & 15.343 & 0.585 & 0.206 & 4 & 2 & 0.729 & 0.715 \\
\hline R8 & $11.529 \pm 5.814$ & 21.517 & 0.636 & 0.408 & 3 & 2 & 0.465 & 0.418 \\
\hline R9 & $12.536 \pm 6.214$ & 10.756 & 0.279 & 0.200 & 2 & 2 & 0.738 & 0.664 \\
\hline R10 & $12.633 \pm 5.442$ & 33.041 & 1.116 & 0.143 & 2 & 2 & 0.813 & 0.746 \\
\hline R11 & $14.295 \pm 7.728$ & 40.646 & 0.680 & 0.326 & 3 & 2 & 0.572 & 0.540 \\
\hline R12 & $16.213 \pm 9.111$ & 72.900 & 0.878 & 0.083 & 3 & 2 & 0.891 & 0.845 \\
\hline $\mathbf{R 1 3}$ & $23.168 \pm 10.041$ & 123.809 & 1.228 & 0.446 & 2 & 2 & 0.415 & 0.377 \\
\hline R14 & $25.078 \pm 9.089$ & 74.857 & 0.906 & 0.278 & 3 & 2 & 0.636 & 0.595 \\
\hline \multicolumn{9}{|l|}{$\overline{M 5}$} \\
\hline R1 & $-0.059 \pm 0.070$ & $0 . \overline{008}$ & 1.687 & $0 . \overline{014}$ & $\overline{1}$ & $\overline{2}$ & 0.986 & 0.986 \\
\hline $\mathbf{R 2}$ & $3.783 \pm 7.291$ & 35.297 & 0.664 & 0.049 & 1 & 2 & 0.951 & 1.016 \\
\hline
\end{tabular}

Tabela 6.59: Síntese das medidas avaliadas para o conjunto de dados Permeability-Cenáriol. 


\begin{tabular}{|c|c|c|c|c|c|c|c|c|}
\hline \multirow{2}{*}{$\begin{array}{r}\# \\
\text { Regra }\end{array}$} & \multicolumn{4}{|c|}{ Precisūo } & \multicolumn{2}{|c|}{ Compreensibilidude } & \multicolumn{2}{|c|}{ Interessubilidude } \\
\hline & Média $\pm D P$ & MSE & NMSE & MADn & \# Cond. & \# Pred. & Ganho & $Q$ \\
\hline \multicolumn{9}{|l|}{ Cubist } \\
\hline $\mathbf{R I}$ & $16.669 \pm 11.898$ & 115.338 & 0.815 & 0.301 & 3 & 2 & 0.699 & 0.699 \\
\hline R2 & $0.030 \pm 0.914$ & 0.831 & 0.994 & 0.004 & 1 & 1 & 0.995 & 4.221 \\
\hline R3 & $0.278 \pm 1.740$ & 2.992 & 0.989 & 0.011 & 3 & 1 & 0.985 & 3.225 \\
\hline R4 & $3.256 \pm 6.132$ & 36.424 & 0.969 & 0.175 & 3 & 1 & 0.750 & 1.854 \\
\hline R5 & $9.372 \pm 5.610$ & 16.806 & 0.534 & 0.104 & 2 & 2 & 0.851 & 1.019 \\
\hline R6 & $15.257 \pm 11.778$ & 124.103 & 0.895 & 0.091 & 2 & 2 & 0.870 & 1.868 \\
\hline \multicolumn{9}{|l|}{$\overline{M 5}$} \\
\hline $\mathbf{R 1}$ & $1.645 \pm 4.856$ & 23.527 & 0.998 & 0.011 & 5 & 1 & 0.989 & $\overline{0.989}$ \\
\hline R2 & $-0.101 \pm 0.112$ & 0.014 & 1.090 & 0.249 & $\mathbf{1}$ & 1 & 0.748 & 1.000 \\
\hline R3 & $0.339 \pm 1.415$ & 1.979 & 0.988 & 0.017 & 4 & 1 & 0.983 & 1.101 \\
\hline R4 & $3.408 \pm 5.095$ & 25.773 & 0.993 & 0.032 & 5 & 1 & 0.967 & 0.902 \\
\hline R5 & $11.125 \pm 3.412$ & 11.722 & 1.007 & 0.852 & 3 & 2 & 0.139 & 0.117 \\
\hline R6 & $12.412 \pm 10.846$ & 95.508 & 0.812 & 0.240 & 2 & 2 & 0.758 & 0.886 \\
\hline
\end{tabular}

Tabela 6.60: Síntese das medidas avaliadas para o conjunto de dados Permeability-Cenário2.

\begin{tabular}{|c|c|c|c|c|c|c|c|c|}
\hline \multirow{2}{*}{$\begin{array}{r}\# \\
\text { Regra }\end{array}$} & \multicolumn{4}{|c|}{ Precistäo } & \multicolumn{2}{|c|}{ Compreensibilidade } & \multicolumn{2}{|c|}{ Interessubilidude } \\
\hline & Média \pm DP & MSE & NMSE & MADn & \# Cond. & \# Pred. & Ganho & $\mathbf{Q}$ \\
\hline \multicolumn{9}{|l|}{ Cubist } \\
\hline$\overline{\mathbf{R}} \mathbf{1}$ & $16.073 \pm 11.929$ & $116.33 \overline{9}$ & $\overline{0.818}$ & 0.303 & 3 & 2 & $\overline{0.697}$ & 0.697 \\
\hline R2 & $0.026 \pm 0.794$ & 0.626 & 0.993 & 0.003 & 1 & 1 & 0.996 & 5.310 \\
\hline R3 & $0.253 \pm 2.085$ & 4.303 & 0.990 & 0.016 & 3 & 1 & 0.977 & 3.624 \\
\hline R4 & $2.775 \pm 6.088$ & 36.057 & 0.973 & 0.164 & 3 & 1 & 0.765 & 1.804 \\
\hline R5 & $8.469 \pm 5.695$ & 17.060 & 0.526 & 0.366 & 2 & 2 & 0.475 & 0.807 \\
\hline $\mathrm{R} 6$ & $15.852 \pm 11.736$ & 123.033 & 0.893 & 0.094 & 2 & 2 & 0.866 & 1.870 \\
\hline \multicolumn{9}{|l|}{$\overline{M 5}$} \\
\hline RI & $-0.061 \pm 0.072$ & 0.006 & 1.092 & 0.272 & 1 & 1 & 0.728 & 0.728 \\
\hline R2 & $4.450 \pm 8.328$ & 42.966 & 0.620 & 0.118 & 1 & 1 & 0.838 & 0.941 \\
\hline
\end{tabular}

Tabela 6.61: Síntese das medidas avaliadas para o conjunto de dados Permeability-Cenário3.

Para o conjunto de dados do PMGRN, divivido em 3 partes nas Tabelas 6.62, 6.63 e 6.64 podem ser observadas informações a respeito de medidas de precisão, compreensibilidade e interessabilidade. Sendo que de acordo com a medida de interessabilidade, as regras R14 e R1 da Tabela 6.62, R5 e R1 da Tabela 6.63 e as R4 e R2 da Tabela 6.64 consistem nas regras mais interessantes do conjunto de dados do PMGRN. 


\begin{tabular}{|c|c|c|c|c|c|c|c|c|}
\hline \multirow{2}{*}{$\begin{array}{r}\# \\
\text { Regra }\end{array}$} & \multicolumn{4}{|c|}{ Precisżo } & \multicolumn{2}{|c|}{ Compreensibilidade } & \multicolumn{2}{|c|}{ Interessabilidade } \\
\hline & Média \pm DP & MSE & NMSE & MADn & \# Cond. & \# Pred. & Ganho & $\mathbf{Q}$ \\
\hline \multicolumn{9}{|l|}{ Cubist } \\
\hline$\overline{\mathrm{Rl}}$ & $221.451 \pm 19.707$ & 294.519 & 0.758 & 0.326 & 5 & 3 & 0.674 & 0.674 \\
\hline $\mathrm{R} 2$ & $171.491 \pm 19.246$ & 230.994 & 0.624 & 0.033 & 2 & 3 & 0.950 & 1.008 \\
\hline R3 & $185.069 \pm 14.827$ & 145.521 & 0.662 & 0.183 & 3 & 3 & 0.729 & 1.037 \\
\hline R4 & $202.774 \pm 18.072$ & 253.849 & 0.777 & 0.087 & 3 & 3 & 0.871 & 0.849 \\
\hline R5 & $205.744 \pm 18.973$ & 285.274 & 0.792 & 0.173 & 4 & 3 & 0.743 & 1.596 \\
\hline R6 & $206.148 \pm 18.532$ & 193.091 & 0.562 & 0.136 & 3 & 3 & 0.799 & 1.810 \\
\hline R7 & $210.238 \pm 14.607$ & 155.538 & 0.729 & 0.186 & 4 & 3 & 0.724 & 1.093 \\
\hline $\mathbf{R} 8$ & $223.247 \pm 16.773$ & 218.588 & 0.777 & 0.043 & 3 & 3 & 0.936 & 1.781 \\
\hline R9 & $234.353 \pm 16.010$ & 240.062 & 0.937 & 0.190 & 4 & 3 & 0.718 & 1.168 \\
\hline R10 & $238.233 \pm 14.931$ & 198.600 & 0.891 & 0.249 & 4 & 3 & 0.630 & 0.938 \\
\hline R11 & $239.499 \pm 19.352$ & 338.956 & 0.905 & 0.151 & 2 & 3 & 0.776 & 1.042 \\
\hline R12 & $241.316 \pm 18.334$ & 323.676 & 0.963 & 0.017 & 5 & 3 & 0.975 & 1.163 \\
\hline RI3 & $256.713 \pm 20.420$ & 288.976 & 0.693 & 0.282 & 3 & 3 & 0.582 & 1.330 \\
\hline R14 & $274.006 \pm 24.216$ & 331.271 & 0.565 & 0.045 & 4 & 3 & 0.934 & 2.398 \\
\hline R15 & $316.758 \pm 28.202$ & 429.149 & 0.540 & 0.263 & 2 & 3 & 0.610 & 0.722 \\
\hline \multicolumn{9}{|l|}{ M5 } \\
\hline $\mathrm{R} 1$ & $234.233 \pm 36.543$ & 278.753 & 0.209 & 0.013 & 1 & 3 & 0.987 & 0.987 \\
\hline
\end{tabular}

Tabela 6.62: Síntese das medidas avaliadas para o conjunto de dados PMGRN455-sexoM.

\begin{tabular}{|c|c|c|c|c|c|c|c|c|}
\hline \multirow{2}{*}{$\begin{array}{r}\# \# \\
\text { Regra }\end{array}$} & \multicolumn{4}{|c|}{$\overline{\text { Precisūo }}$} & \multicolumn{2}{|c|}{ Compreensibilidade } & \multicolumn{2}{|c|}{ Interessubilidade } \\
\hline & Média $\pm D P$ & MSE & NMSE & MADn & \# Cond. & \# Pred. & Ganbo & Q \\
\hline \multicolumn{9}{|l|}{ Cubist } \\
\hline $\mathrm{R} 1$ & $243.666 \pm 30.691$ & 308.820 & 0.328 & 0.003 & 4 & $\overline{3}$ & 0.998 & $0 . \overline{998}$ \\
\hline R2 & $208.021 \pm 28.590$ & 355.887 & 0.435 & 0.019 & 2 & 3 & 0.981 & 0.913 \\
\hline $\mathrm{R3}$ & $230.728 \pm 21.104$ & 256.432 & 0.576 & 0.218 & 3 & 3 & 0.782 & 0.700 \\
\hline R4 & $231.954 \pm 29.332$ & 216.215 & 0.251 & 0.006 & 3 & 3 & 0.994 & 0.948 \\
\hline R5 & $254.909 \pm 26.593$ & 260.426 & 0.368 & 0.009 & 3 & 3 & 0.991 & 1.152 \\
\hline R6 & $308.547 \pm 24.385$ & 296.094 & 0.498 & 0.095 & 2 & 3 & 0.905 & 0.917 \\
\hline R7 & $321.648 \pm 40.912$ & 351.544 & 0.210 & 0.110 & 2 & 3 & 0.890 & 0.864 \\
\hline \multicolumn{9}{|l|}{$\mathcal{M 5}$} \\
\hline R1 & $232.289 \pm 26.765$ & 271.922 & 0.380 & 0.037 & 1 & 3 & 0.963 & 0.963 \\
\hline $\mathrm{R} 2$ & $293.182 \pm 33.786$ & 315.224 & 0.276 & 0.054 & 1 & 3 & 0.944 & 0.959 \\
\hline
\end{tabular}

Tabela 6.63: Síntese das medidas avaliadas para o conjunto de dados PMGRN455-sexoF.

\begin{tabular}{|c|c|c|c|c|c|c|c|c|}
\hline \multirow{2}{*}{$\begin{array}{r}\# \\
\text { Regra }\end{array}$} & \multicolumn{4}{|c|}{ Precisǘo } & \multicolumn{2}{|c|}{ Compreensibilidade } & \multicolumn{2}{|c|}{ Interessabilidade } \\
\hline & Média \pm DP & MSE & NMSE & MADn & \# Cond. & \# Pred. & Gunho & $\mathbf{Q}$ \\
\hline \multicolumn{9}{|l|}{ Cubist } \\
\hline $\mathrm{RI}$ & $187.431 \pm 28.137$ & 316.908 & 0.603 & 0.238 & 4 & 3 & 0.762 & $\overline{0.762}$ \\
\hline R2 & $220.212 \pm 26.540$ & 292.699 & 0.370 & 0.076 & 2 & 3 & 0.900 & 0.939 \\
\hline R3 & $200.246 \pm 19.806$ & 256.650 & 0.654 & 0.130 & 3 & 3 & 0.829 & 0.868 \\
\hline R4 & $296.299 \pm 36.118$ & 254.572 & 0.361 & 0.158 & 3 & 3 & 0.793 & 2.157 \\
\hline R5 & $279.710 \pm 23.469$ & 256.182 & 0.584 & 0.021 & 3 & 3 & 0.973 & 1.935 \\
\hline R6 & $206.866 \pm 22.932$ & 700.438 & 0.777 & 0.291 & 2 & 3 & 0.619 & 0.590 \\
\hline R7 & $234.349 \pm 20.953$ & 304.221 & 0.552 & 0.030 & 3 & 3 & $0.96 \mathrm{I}$ & 1.587 \\
\hline $\mathbf{R} 8$ & $256.818 \pm 30.024$ & 346.753 & 0.266 & 0.064 & 3 & 3 & 0.917 & 1.756 \\
\hline \multicolumn{9}{|l|}{$\mathcal{M} 5$} \\
\hline$\overline{\mathrm{R} 1}$ & $221.432 \pm 25.905$ & 260.174 & 0.388 & 0.114 & $\bar{I}$ & 3 & 0.886 & 0.886 \\
\hline $\mathrm{R} 2$ & $285.127 \pm 33.063$ & 349.447 & 0.320 & 0.042 & 1 & 3 & 0.953 & 0.931 \\
\hline
\end{tabular}

Tabela 6.64: Síntese das medidas avaliadas para o conjunto de dados PMGRN455-total.

\subsection{Considerações Finais}

Neste capítulo foram descritos experimentos realizados utilizando os algoritmos Cubist e $\mathcal{M} 5$ em diferenites conjuntos de dados com diferentes características. Para avaliar o conhecimento ex- 
traído nos experimentos realizados foi utilizado o ambiente $\mathcal{R} \mathcal{R}_{\text {Evaluation }}$.

Todas as medidas de precisão, compreensibilidade e interessabilidade para avaliar o conhe-

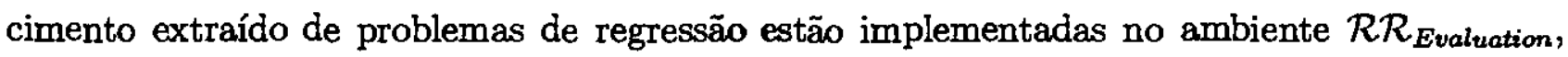
podendo obter assim, informações dessas medidas automaticamente.

Além de demonstrar a utilização da ferramenta, todos os experimentos tinham como objetivo a verificação das regras mais interessantes, segundo os critérios de interessabilidade. Essa interessabilidade deve levar em conta também a precisão das regras, sendo que os valores obtidos com as medidas de MSE, NMSE e MAD nas regras do $\mathcal{M} 5$ foram, no geral, mais precisas do que as geradas pelo Cubist.

Ressalta-se a necessidade de estudar outras formas para avaliar a compreensibilidade do conhecimento extraído uma vez que nos experimentos realizados as medidas utilizadas não se mostraram satisfatórias.

Uma descrição mais detalhada dos experimentos realizados, bem como do $\mathcal{R}_{\mathcal{R}_{\text {Evaluation, }} \text {, podem }}$ ser encontrados em [Nagai and Rezende, 2000]. 


\section{Capítulo 7}

\section{Conclusões e Trabalhos Futuros}

Muitas pesquisas têm sido realizadas na área de Data Mining preditivo, entretanto, ainda existe uma série de questões em aberto, especialmente com relação à avaliação do conhecimento extraído.

Grande parte das pesquisas que vêm sendo realizadas no campo da avaliação do conhecimento extraído utilizando o processo de Data Mining estão direcionadas a problemas de classificação (problemas que envolvem atributos-meta categóricos). Entretanto, a maioria dos problemas do mundo real é de natureza contínua. Esses são os problemas de regressão no qual a previsão é realizada utilizando uma função matemática. Devido à natureza do problema, a avaliação do conhecimento nesses problemas de regressão é ainda mais complexa.

Neste trabalho foram abordadas algumas questōes referentes ao processo de Data Mining preditivo em problemas de regressão, tais como: os tipos de aprendizado e linguagens de representações dos padrões mais utilizados, e algumas das medidas para avaliação de conhecimento adequadas a essa classe de problemas.

Como mencionado, dependendo do tamanho do conjunto de exemplos é encontrada uma grande quantidade de padrões, tornando o processo de avaliação do conhecimento uma atividade custosa. Dessa forma, torna-se necessário um ambiente de avaliação capaz de manipular essa quantidade de regras e retornar ao especialista/usuário do domínio, as regras consideradas mais "interessantes".

Assim, foi aqui proposto, o ambiente $\mathcal{R}_{\mathcal{R}_{\text {Evaluation }}}$ com objetivo de apoiar o usuário do processo Data Mining na avaliação dos resultados encontrados quando o domínio trata-se de problemas de regressão. Para avaliar o desempenho o ambiente utiliza as medidas MSE, MAD e NMSE 
e para verificar a compreensiblidade foi utilizado o número de condiçōes da regra e da função matemática envolvida. Já para calcular a interessabilidade, as medidas GanhoMAD, LC e Q foram usadas.

A versāo atual do sistema avalia o conhecimento extraído quando sāo utilizados os algoritmos Cubist e M5. O conhecimento obtido pelo Cubist é incorporado diretamente ao ambiente na base $\mathrm{B}_{R R}$, enquanto que para o $\mathcal{M} 5$ é necessário traduzir a árvore de regressão para o formato da base de regras $\mathrm{B}_{R R}$. Para resolver este problema no ambiente $\mathcal{R} \mathcal{R}_{\text {Evaluation }}$ existe um módulo de conversão denominado de $\mathcal{M} 5_{2}$ Rules que é responsável por esta tradução.

Como já ressaltado, o $\mathcal{R R}_{\text {Evaluation }}$ pode ser estendido para avaliar regras de regressão geradas por outros algoritmos, sendo necessária a implementação de outros módulos de conversão para a $\mathrm{B}_{R R}$. Esses módulos de conversão devem ser responsáveis pela tradução dos padrões extraídos por esses algoritmos para o formato da $B_{R R}$ cuja sintaxe foi definida e mostrada na Tabela 5.4 on page 45. Vale lembrar também que o conjunto de dados de teste deve seguir a sintaxe definida na Tabela 5.2 e 5.3 .

O $\mathcal{R} \mathcal{R}_{\text {Evaluation }}$ é um dos projetos que estão integrados a um projeto mais geral de desenvolvimento de um Ambiente Computacional para Extração de Conhecimento - DisCover, em andamento no Laboratório de Inteligência Computacional (LABIC) no ICMC-USP/São Carlos. Esse ambiente visa a combinação dos diversos trabalhos realizados pelo grupo a um único ambiente integrado de extração de conhecimento de bases de dados. No Discover são utilizados algoritmos de AM implementados pela comunidade bem como módulos com finalidades específicas desenvolvidos pelos pesquisadores deste projeto. Tal ambiente é capaz de acessar os dados na forma de atributo-valor ou na forma de texto. Os resultados dos algoritmos de extração de padrões ou modelos podem ser avaliados de diversas formas, entre elas precisão e qualidade. $\mathrm{O}$ ambiente $\mathcal{R} \mathcal{R}_{\text {Evaluation }}$ é o responsável pela avaliação dos resultados quando o domínio de conhecimento trata-se de problemas de regressão.

Neste trabalho, além do desenvolvimento do ambiente $\mathcal{R} \mathcal{R}_{\text {Evaluation }}$ foram apresentados diversos experimentos, em diferentes domínios e com diferentes características, utilizando os algoritmos Cubist e M5. Foram envolvidos seis diferentes domínios, sendo um deles um estudo de casos em uma base de dados do mundo real, e para a avajiação dos resultados utilizou-se o ambiente $\mathcal{R} \mathcal{R}_{\text {Evaluation. }}$. Os resultados desses experimentos mostram a viabilidade do uso do ambiente $\mathcal{R}_{\text {Evaluation }}$ como ferramenta de apoio ao especialista na avaliação dos resultados do processo de Data Mining.

Como sugestão são vários os trabalhos futuros a serem realizados, dentre eles, destaca-se: 


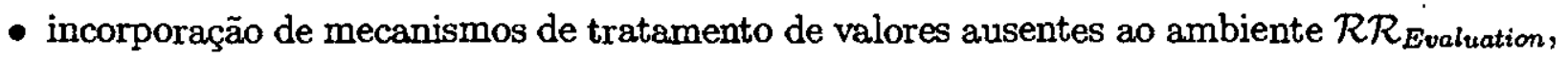
para, entre outros, evitar problemas como os tratados no experimento com o conjunto de dados Boston Housing;

- em relação ao DISCOVER, adicionar o conhecimento extraído de problemas de regressão na base de dados MySQL existente, bem como estruturar uma tabela específica para armazenar as regras de regressão.

- com relação aos experimentos já realizados, ressalta-se a necessidade de calcular a precisão dos resultados dos conjuntos de dados utilizando o $\mathcal{M} 5$, pois os erros observados na predição são apenas aparentes. Dessa forma, é necessária uma validação experimentos utilizando, por exemplo, k-fold cross-validation;

- avaliar o conhecimento obtido para problemas de regressão quando utilizada a Programação Lógica Indutiva no conjunto de dados de treinamento. Desta forma, o conhecimento do especialista do domínio pode ser adicionado para refutar os padrões espúrios ou sem nenhum valor; 


\section{Referências Bibliográficas}

[Baranauskas and Monard, 2000] Baranauskas, J. A. and Monard, M. C. (2000). Reviewing some machine learning concepts and methods. Relatório Técnico 102, Instituto de Ciências Matemáticas e de Computação, São Carlos, SP - Brazil.

[Batista, 1997] Batista, G. E. A. P. A. (1997). Um Ambiente de Avaliação de Algoritmos de Aprendizado de Máquina utilizando Exemplos. Master's thesis, ICMC-USP. Dissertação de Mestrado, ICMC-USP.

[Blake et al., 1998] Blake, C., Keogh, E., and Merz, C. (1998). UCI repository of machine learning databases. http://www .ics.uci.edu/“mlearn/MLRepository.html.

[Breiman and Friedman, 1985] Breiman, L. and Friedman, J. (1985). Estimating optimal transformations in multiple regression and correlation. Journal of the American Statistical Association, 80:580-619.

[Breiman et al., 1984] Breiman, L., Friedman, J. H., Olshen, R. A., and Stone, C. J. (1984). Classification and Regression Trees. Wadsworth International, Monterey, CA.

[Cheng, 1998] Cheng, S. (1998). Statistical approaches to predictive modeling in large databases. Master's thesis, Simon Fraser University, British Columbia, Canada.

[Clark and Niblett, 1989] Clark, P. and Niblett, T. (1989). The CN2 induction algorithm. Machine Learning, 3:261-283.

[Craven and Shavlik, 1999] Craven, M. and Shavlik, J. (1999). Rule extraction: Where do we go from here? http://www.cs.cmu.edu/" craven/rule-ex-position.ps - 10/06/2000.

[Craven, 1996] Craven, M. W. (1996). Extracting Comprehensible Models from Trained Neural Networks. $\mathrm{PhD}$ thesis, University of Wisconsin, Madison.

[Dougherty et al., 1995] Dougherty, J., Kohavi, R., and Sahami, M. (1995). Supervised and unsupervised discretization of continuous features. In Proc. 12th International Conference on Machine Learning, pages 194-202. Morgan Kaufmann. 
[Elder and Pregibon, 1996] Elder, J. and Pregibon, D. (1996). A statistical perspective on knowledge discovery in databases. In Fayyad, U. M., Piatetsky-Shapiro, G., Smyth, P., and Uthurusamy, R., editors, Advances in Knowledge Discovery and Data Mining, pages 83-115. AAAI/MIT Press.

[Fayyad et al., 1996a] Fayyad, U., Piatetsky-Shapiro, G., and Smyth, P. (1996a). Knowledge discovery and data mining: Towards a unifying framework. In Proceedings of the Second International Conference on Knowledge Discovery and Data Mining (KDD-96), Portland, Oregon. AAAI Press. Disponível em: http://www-aig.jpl.nasa.gov/kdd96.

[Fayyad, 1996a] Fayyad, U. M. (1996a). Data Mining and Knowledge Discovery - Making Sense Out of Data. IEEE Expert-Intelligent Systems \& Their Applications, 11:20-25.

[Fayyad, 1996b] Fayyad, U. M. (1996b). The KDDProcess for Extracting Useful Knowledge and Data Mining. Communications of the ACM, 39(11):27-34.

[Fayyad et al., 1996b] Fayyad, U. M., Piatesky-Shapiro, G., Smyth, P., and Uthurusamy, R. (1996b). From Data Mining to Knowledge Discovery in Databases. American Association for Artificial Intelligence, pages 37-54.

[Fayyad et al., 1996c] Fayyad, U. M., Piatetsky-Shapiro, G., Smyth, P., and Uthurusamy, R., editors (1996c). Advances in Knowledge Discovery and Data Mining. AAAI Press/MIT Press.

[Félix, 1998] Félix, L. C. M. (1998). Data mining no processo de extração de conhecimento de bases de dados. Master's thesis, Instituto de Ciências Matemáticas e de Computação. Dissertação de Mestrado, ICMC-USP.

[Félix et al., 1998a] Félix, L. C. M., Rezende, S. O., Doi, C. Y., de Paula, M. F., and Romanato, M. J. (1998a). $\mathcal{M L C}^{++}$Biblioteca de Aprendizado de Máquina em $\mathcal{C}^{++}$. Technical Report 72, ICMC-USP.

[Félix et al., 1998b] Félix, L. C. M., Rezende, S. O., Monard, M. C., and Caulkins, C. W. (1998b). Transforming a regression problem into a classification problem observing the discretization process. Technical Report 76, ICMC-USP.

[Félix et al., 1999] Félix, L. C. M., Rezende, S. O., Monard, M. C., and Caulkins, C. W. (1999). Some Experiences with the Discretization Process: from Regression to Classification. In Proceedings of the Argentine Symposium on Artificial Intelligence, pages 155-166, Buenos Aires. 
[Félix et al., 2000] Félix, L. C. M., Rezende, S. O., Monard, M. C., and Caulkins, C. W. (2000). Transforming a Regression Problem into a Classification Problem Using Hybrid Discretization. In Computacion y Sistemas. CIC - IPN. (in print).

[Frank et al., 1998] Frank, E., Wang, Y., Inglis, S., Holmes, G., and Witten, I. H. (1998). Using model trees for classification. Machine Learning, 32:63-76.

[Freitas, 1998] Freitas, A. A. (1998). A multi-criteria approach for the evaluation of rule interestingness. In Ebecken, N., editor, Proceedings of the International Conference on Data Mining, pages 7-20, Rio de Janeiro.

[Friedman, 1990] Friedman, J. H. (1990). Multivariate adaptive regression splines. Technical Report. http://www.salford-systems.com/MARS.pdf.

[Fu and Han, 1995] Fu, Y. and Han, J. (1995). Meta-rule-guided mining of association rules in relational databases. In Proceedings 1995 International Workshop on Knowledge Discovery and Deductive and Object-Oriented Databases (KDOOD'95), pages 39-46, Singapore.

[Haykin, 1994] Haykin, S. (1994). Neural Networks A Comprehensive Foundation. Macmillan College Publishing Company, Inc.

[Heckerman, 1997a] Heckerman, D. (1997a). Bayesian Networks for Data Mining. Data Mining and Knowledge Discovery, 1:79-119.

[Heckerman, 1997b] Heckerman, D. (1997b). Bayesian networks for knowledge discovery. Data Mining and Knowledge Discovery, 1(1):79-119.

[Horst, 1999] Horst, P. S. (1999). Avaliação do conhecimento adquirido por algoritmos de aprendizado de máquina utilizando exemplos. Master's thesis, ICMC-USP. Dissertação de Mestrado, ICMC-USP.

[Karalic, 1992] Karalic, A. (1992). Employing linear regression in regression tree leaves. Technical Report IJS DP-6450, Jozef Stefan Institute, Ljubljana, Slovenia.

[Kerber, 1991] Kerber, R. (1991). Learning classification from examples. In Proceedings AAAI Workshop on Knowledge Discovery in Databases. Springer-Verlag.

[Kibler and Aha, 1988] Kibler, D. and Aha, D. (1988). Instance based prediction of real-valued attributes. In Proceedings of the CSCSI (Canadian AI) Conference.

[Klemettinen et al., 1994] Klemettinen, M., Mannila, H., Ronkainen, P., Toivonen, H., and Verkamo, A. I. (1994). Finding interesting rules from large sets of discovered association 
rules. In Proceedings of the Third International Conference on Information and Knowledge Management, pages 401-407, Gaithersburg, Maryland.

[Kohavi et al., 1994] Kohavi, R., John, G., Long, R., Manley, D., and Pfleger, K. (1994). $\mathcal{M L C}^{++}$: A machine learning library in $\mathrm{C}^{++}$. Tools with Artificial Intelligence, IEEE Computer Society Press, pages 740-743. http://www.sgi.com/Technology/mlc.

[Kohavi et al., 1997] Kohavi, R., Sommerfield, D., and Dougberty, J. (1997). Data mining using $\mathcal{M L C}^{++}$: A machine learning library in $\mathcal{C}^{++}$. International Journal on Artificial Intelligence Tools, $0(0)$.

[Kubat et al., 1998] Kubat, M., Bratko, I., and Michalski, R. S. (1998). A Review of Machine Learning Methods. John Wiley \& Sons Ltd., West Sussex, England.

[Lee, 2000] Lee, H. D. (2000). Seleçāo e construção de Features relevantes para o aprendizado de máquina. Master's thesis, ICMC-USP, São Carlos - SP, Brasil.

[Ling and Wang, 1997] Ling, C. X. and Wang, H. (1997). Computing optimal attribute weight setting for nearest neighbor algorithms. Artificial Intelligence Review, pages 255-272.

[Liu and Hsu, 1996] Liu, B. and Hsu, W. (1996). Post-analysis of learned rules. In Proceedings of the Thirteenth National Conference on Artificial Intelligence (AAAI-96), pages 828-834. http://www. comp.nus.edu.sg/ /iub/publications/aaai96.int.ps.

[Lôbo et al., 1999] Lôbo, R. B., Bezerra, L. A., Oliveira, H. N., and de los Reyes, A. (1999). Avaliação genética de animais jovens, touros e matrizes - sumário 1999. http://www_gen. fmrp.usp.br.

[Merz, 1998] Merz, C. J. (1998). Classification and Regression by Combining Models. $\mathrm{PhD}$ thesis, University of California, Irvine. Disponível em: http://ww .ics.uci. edu:80/ cmerz/thesis.ps.

[Murthy et al., 1994] Murthy, S. K., Kasif, S., and Salzberg, S. L. (1994). A system for induction of oblique decision trees. Journal of Artificial Intelligence Research, 2(1):1-32. http://wwr.cs. jhu. edu/ salzberg/jair94.ps.

[Nagai and Rezende, 2000] Nagai, W. A. and Rezende, S. O. (2000). Ambiente para avaliação de conhecimento em problema de regressão- $\mathcal{R R}_{\text {Evaluation. Relatório técnico do ICMC-USP }}$ (em preparação).

'Padilha and Rezende, 1999] Padilha, T. P. P. and Rezende, S. O. (1999). Algoritmos de aprendizado de máquina. Technical report, ICMC-USP (em andamento). 
[Piatetsky-Shapiro, 1991] Piatetsky-Shapiro, G. (1991). Discovery, analysis and presentation of strong rules. In Piatetsky-Shapiro, G. and Frawley, W. J., editors, Knowledge Discovery in Databases, pages 229-248.

[Piatetsky-Shapiro and Matheus, 1994] Piatetsky-Shapiro, G. and Matheus, C. (1994). The interestingness of deviations. KDD-94, pages 23-36.

[Quinlan, 1986] Quinlan, J. R. (1986). Simplifying decision trees. In Proceedings Workshop on Knowledge Acquisition for Knowledge-based Systems.

[Quinlan, 1992] Quinlan, J. R. (1992). Learning with continuous classes. In Proceedings Australian Joint Conference on Artificial Intelligence, pages 343-348. World Scientific.

[Quinlan, 1993a] Quinlan, J. R. (1993a). C4.5: Programs for Machine Learming. Morgan Kaufmann Publishers, Inc., San Mateo, CA.

[Quinlan, 1993b] Quinlan, J. R. (1993b). Combining instance-based and model-based learning. In Proceedings on the Tenth Intermational Conference of Machine Learning, pages 236-243, University of Massachusetts, Amherst. Morgan Kaufmann.

[Ram, 1990] Ram, A. (1990). Knowledge Goals: A Theory of Interestingness. In Proceedings of the Twelvth Annual Conference of the Cognitive Science Society, pages 206-214, Cambridge, MA,. http://www.cc.gatech. edu/faculty/ashwin/papers/er-90-02.ps.Z.

[Rezende et al., 1998] Rezende, S. O., Oliveira, R. B. T., Félix, L. C. M., and da Rocha, C. A. J. (1998). Visualization for knowledge discovery in database. In Ebecken, N.F.F. (ed.) Data Mining. WIT Press, England, pages 81-95.

[Rocha, 1998] Rocha, C. A. J. (1998). Processo de Extração de Conhecimento de Base de Dados Considerando a Incorporação de Conhecimento de Fundo e Tratamento de Dados Incompletos. Exame de Qualificação, ICMC-USP/São Carlos.

[Rogers et al., 1995] Rogers, S. J., Chen, H. C., Kopaska-Merkel, D. C., and Fang, J. (1995). Predicting permeability from porosity using artificial neural networks. American Association of Petroleum Geologists Bulletin, 79:1786-1797.

[Rulequest-Research, 2000] Rulequest-Research (2000). Rulequest Research - Data Mining Tools. http://wrw. rulequest. com.

[Silberschatz and Tuzhilin, 1995] Silberschatz, A. and Tuzhilin, A. (1995). On subjective measures of interestingness in knowledge discovery. Proceedings of the First International Conference on Knowledge Discovery and Data Mining - KDD-95, pages 275-281. 
[Silberschatz and Tuzhilin, 1996] Silberschatz, A. and Tuzhilin, A. (1996). What makes patterns interesting in knowledge discovery patterns. IEEE Transactions on Knowledge and Data Engineering, 8(6):970-974.

[Toivonen et al., 1995] Toivonen, H., Klemettinen, M., Ronkainen, P., H (1995). Prunning and grouping discovered association rules. In MLNet Workshop. on Statistics, Machine Learning and Discovery in Databases, Crete, Greece.

[Torgo, 1995] Torgo, L. (1995). Data fitting with rule-based regression. In Proceedings of the workshop on Artificial Intelligence Techniques (AIT'95), Brno, Czech Republic. J. Zizka \& P. Brazdil.

[Torgo, 1997] Torgo, L. (1997). Functional models for regression tree leaves. Proceedings of the International Machine Learning Conference (ICML-97). http://www.ncc.up.pt/ "Itorgo --- 26/10/1999.

[Torgo and ao Gama, 1997] Torgo, L. and ao Gama, J. (1997). Search-based class discretization. In Proceedings of the European Conference on Machine Learning (ECML-97), Prague, Czech Republic. Lecture Notes in Artificial Intelligence 1224, Springer Verlag. http://www.ncc.up.pt/ Itorgo/Papers/SCD.ps.gz.

[Torgo, 1999] Torgo, L. F. R. A. (1999). Inductive Learning of Tree-Based Regression Models. $\mathrm{PhD}$ thesis, Faculdade de Ciências da Universidade do Porto. Disponível em: http://www.ncc.up.pt/ Itorgo/ $/ \mathrm{phD} /$.

[Uysal and Güvenir, 1999] Uysal, I. and Güvenir, H. A. (1999). An overview of regression techniques for knowledge discovery. The Knowledge Engineering Review, 14(4):319-340.

[Vanderberg, 2000] Vanderberg, H. (2000). Mineset 3.0 enterprise edition interface guide. Silicon Graphics Inc. http://techpubs.sgi.com/Iibrary/manuals/4000/007-4005-001/ pdf/007-4005-\%001.pdf - 11/07/2000.

[Wang and Witten, 1997] Wang, Y. and Witten, I. H. (1997). Induction of model trees for predicting continuous classes. In Proceedings of the Poster Papers of the European Conference on Machine Learning.

[Weiss and Indurkhya, 1995] Weiss, S. M. and Indurkhya, N. (1995). Rule-based machine learning methods for functional prediction. Journal of Artificial Intelligence Research, 3:383-403. Disponível em: http://www.cs.cmu.edu/afs/cs/project/jair/pub/volume3/weiss95a. ps $-04 / 05 / 2000$. 
[Weiss and Indurkhya, 1998] Weiss, S. M. and Indurkhya, N. (1998). Predictive Data Mining A Pratical Guide. Morgan Kaufmann Publishers, Inc., San Francisco, California.

[Weiss and Kulikowski, 1991] Weiss, S. M. and Kulikowski, C. A. (1991). Computer Systems That Learn: Classification and Prediction Methods from Statistics, Neural Nets, Machine Learning, and Expert Systems. Morgan Kaufmann, San Mateo, California.

[Witten and Frank, 1999] Witten, I. H. and Frank, E. (1999). Data Mining: Practical Machine Learning Tools and Techniques with Java Implementations. Morgan Kaufmann. http://www.cs.waikato.ac.nz/ ml/weka/book.html. 